\title{
Towards predictive flavour models in SUSY SU(5) GUTs with doublet-triplet splitting
}

\author{
Stefan Antusch, ${ }^{a, b}$ Ivo de Medeiros Varzielas, ${ }^{a}$ Vinzenz Maurer, ${ }^{a}$ Constantin Sluka ${ }^{a}$ \\ and Martin Spinrath ${ }^{c}$ \\ ${ }^{a}$ Department of Physics, University of Basel, \\ Klingelbergstr. 82, CH-4056 Basel, Switzerland \\ ${ }^{b}$ Max-Planck-Institut für Physik (Werner-Heisenberg-Institut), \\ Föhringer Ring 6, D-80805 München, Germany \\ ${ }^{c}$ Institut für Theoretische Teilchenphysik, Karlsruhe Institute of Technology, \\ Engesserstraße 7, D-76131 Karlsruhe, Germany \\ E-mail: stefan.antusch@unibas.ch, ivo.de@unibas.ch, \\ vinzenz.maurer@unibas.ch, constantin.sluka@unibas.ch, \\ martin.spinrath@kit.edu
}

ABSTRACT: We discuss how the double missing partner mechanism solution to the doublettriplet splitting problem in four-dimensional supersymmetric SU(5) Grand Unified Theories (GUTs) can be combined with predictive models for the quark-lepton Yukawa coupling ratios at the GUT scale. It is argued that towards this goal a second $\mathrm{SU}(5)$ breaking Higgs field in the adjoint representation is very useful and we discuss all possible renormalizable superpotentials with two adjoint Higgs fields and calculate the constraints on the GUT scale and effective triplet mass from a two-loop gauge coupling unification analysis. Two explicit flavour models with different predictions for the GUT scale Yukawa sector are presented, including shaping symmetries and a renormalizable messenger sector. Towards calculating the rates for proton decay induced by the exchange of colour triplets, the required ClebschGordan coefficients for their couplings are calculated for the possible dimension five and six operators. They are provided in detailed tables in the appendix, together with additional helpful material for GUT flavour model building.

KeYwords: GUT, Beyond Standard Model

ARXIV EPRINT: 1405.6962 


\section{Contents}

1 Introduction 1

2 Strategy 3

2.1 The missing partner mechanism 4

2.2 The double missing partner mechanism 5

$\begin{array}{lll}2.3 & \text { Planck-scale suppressed operators } & 6\end{array}$

2.4 Yukawa coupling ratios 6

2.5 The double missing partner mechanism with an adjoint 8

$\begin{array}{lll}2.6 & \text { Introducing a second adjoint field } & 10\end{array}$

3 Grand unification and the effective triplet mass 11

$\begin{array}{lll}3.1 & \text { Superpotentials with two adjoints of SU(5) } & 13\end{array}$

$\begin{array}{lll}3.1 .1 & \text { Superpotential (a) } & 14\end{array}$

$\begin{array}{lll}3.1 .2 & \text { Superpotential (b) and (c) } & 14\end{array}$

4 Flavour models with DMPM $\quad 17$

$\begin{array}{lll}4.1 \text { A model with diagonal } Y_{d} \text { and } Y_{e} \text { Yukawa matrices } & 18\end{array}$

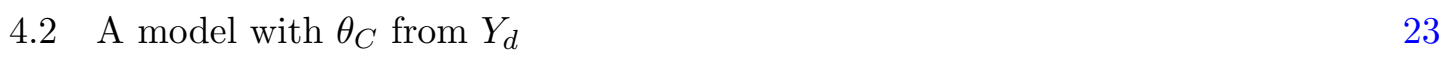

5 Proton decay $\quad 28$

5.1 Proton decay from dimension five operators 29

5.2 Proton decay from dimension six operators 31

$\begin{array}{lll}6 & \text { Summary and conclusions } & 31\end{array}$

A Yukawa coupling ratios including colour triplets 32

B Two-loop RGEs in extensions to the MSSM 38

$\begin{array}{ll}\text { C Discussion of messenger fields } & 40\end{array}$

D Simultaneous R-symmetry and GUT breaking $\quad 41$

\section{Introduction}

Grand Unified Theories (GUTs) offer one of the most attractive extensions of the Standard Model of particle physics (SM), unifying three of the four fundamental forces of nature. Towards a more fundamental theory of nature it would, however, be desirable to also explain the pattern of fermion masses and mixing with its plenty of parameters. Hence, successful models of flavour in GUTs need to address two main issues, firstly, they have to 
achieve a working mechanism for GUT symmetry breaking, including sufficient suppression of proton decay without unacceptably large fine-tuning, and secondly, they should provide correct predictions for the flavour observables, such as the Yukawa coupling ratios and mixing angles.

Existing models which manage to naturally suppress proton decay either predict the unrealistic quark-lepton Yukawa relation $Y_{e}=Y_{d}^{T}$ (e.g. [1]), which may only be viable in the presence of extensive uncontrolled higher order corrections (e.g. [2]) rendering the model non-predictive, or the experimentally disfavoured combination of the Georgi-Jarlskog relations $y_{\mu}=-3 y_{s}$ and $y_{e}=\frac{1}{3} y_{d}$ [3] (as e.g. in [4]), or rely on linear combinations of GUT Yukawa operators (e.g. [5]), which again implies the loss of predictivity. Furthermore, there exists a large number of GUT models which focus on the flavour sector, but do not include the Higgs potential. So while there are several existing models focusing on one of these two concerns, we are not aware of any work to date capable of resolving the two challenges in full detail (and without invoking extra space-time dimensions) in a predictive setup, also given the present rather precise experimental data.

In this paper we employ the framework of supersymmetric (SUSY) GUTs, as in the minimal supersymmetric extension of the SM (MSSM) the gauge couplings unify to a surprising precision. The scale where they unify (about $10^{16} \mathrm{GeV}$ ) is also large enough to sufficiently suppress proton decay from dimension six GUT operators [6]. However, in addition to proton decay mediated by additional heavy gauge bosons, in a GUT model the minimal embedding of the Higgs fields contains additional colour triplets which also lead to baryon number violating operators. Therefore the colour triplets have to be very heavy to suppress proton decay sufficiently or their couplings to the MSSM fields should be very strongly suppressed. Keeping the doublets of the SU(5) Higgs fields light, while generating a high enough mass for the colour triplets constitutes the so-called "DoubletTriplet Splitting (DTS) Problem".

In $\mathrm{SU}(5)$ in four dimensions, a proposed solution to the DTS problem is the "missing partner mechanism" (MPM) [7, 8] or its improved version, the "Double Missing Partner Mechanism" (DMPM) [9] which we will review briefly in the next section. In the existing models referred to above, either the MPM or the DMPM is applied.

Since GUTs not only unify the forces of the SM into a single GUT force, but also the fermions into joint GUT representations, they are indeed a promising starting point for addressing the flavour puzzle. More specifically, GUTs are capable of predicting the ratios between the Yukawa couplings of quarks and leptons at the GUT scale. After their renormalization group evolution to low energies (including supersymmetric 1-loop threshold corrections), these predictions can be compared to the experimental data for quark and lepton masses. As well known, the prediction of minimal SU(5) for the charged lepton and down-type quark Yukawa matrix, the relation $Y_{e}=Y_{d}^{T}$ already mentioned above, is strongly disfavoured by the experimental results on the fermion masses. But also the ubiquitous proposal for more realistic ratios, the above mentioned Georgi-Jarlskog relations, obtained from the introduction of a 45-dimensional Higgs representation of SU(5) and certain assumptions about the Yukawa textures [3], are disfavoured by the recently improved data [10]. 
To arrive at experimentally favoured predictions for GUT scale Yukawa coupling ratios, we employ an alternative approach [11] involving higher-dimensional operators which contain a GUT breaking Higgs field. Due to this, new Clebsch-Gordan (CG) factors appear as Yukawa coupling ratios, with interesting associated implications for the masses and mixing of the fermions, cf. [12, 13] and [14-17]. The main goal of this paper is to show how GUT flavour models featuring these promising quark-lepton mass relations can be combined with a version of the DMPM for solving the DTS problem.

As explicit examples, we will present two models with these properties that are "UV complete" in terms of messenger fields and employ sets of discrete Abelian symmetries (referred to as shaping symmetries) such that only the desired effective GUT operators are generated when the heavy degrees of freedom are integrated out. The two models predict the GUT scale quark lepton Yukawa ratios but are not yet predictive for the fermion mixing parameters (although the experimentally observed values can be fitted by both of the models), so we only view them as existence proofs which show that DTS and predictive Yukawa coupling ratios can indeed be combined. The strategies discussed here, however, provide the tools for the construction of more ambitious GUT models of flavour, which should finally also predict the quark and lepton mixings and CP phases (and include the observed neutrino masses).

The paper is organised as follows: we will start with a brief review of the MPM and DMPM and the recently proposed alternative Yukawa coupling ratios. We will also discuss the implications of replacing the 75-dimensional representation used in the MPM and DMPM with an adjoint 24-dimensional representation of SU(5). This choice is particularly well suited towards combining the DMPM with the novel CG factors. In section 3 we discuss the impact of the additional fields on gauge coupling unification and on their implications for the colour triplet masses. We will especially focus here on the case of superpotentials with two adjoint Higgs representations. We then address the Yukawa sector in section 4, where we describe the above-mentioned two predictive example models. Before we summarise and conclude in section 6 , in section 5 we briefly comment on proton decay, showing it is under control in the proposed class of models. We present additional helpful material for model building in the appendices.

\section{$2 \quad$ Strategy}

In this section we present the general strategy that will be implemented in two example models. We begin our presentation with the review of the MPM and DMPM and the origin of the CG coefficients (coming from higher dimensional operators) used in our models. Afterwards we discuss a modification of the DMPM with a GUT Higgs field in the adjoint representation and follow that with the actual DMPM realization implemented in our models, where a second Higgs field in the adjoint representation is added.

Throughout this section, for illustrative purposes, we consider that the bounds on proton decay rate require the effective mass of the colour triplets to be of at least $M_{T}^{\text {dim=5 }} \approx 10^{17} \mathrm{GeV}$ [18], while the effective mass suppressing dimension six proton decay mediated by the colour triplets is required to be $M_{T}^{\mathrm{dim}=6} \gtrsim 10^{12} \mathrm{GeV}$ [19]. 


\subsection{The missing partner mechanism}

The basic idea of the missing partner mechanism (MPM) is the introduction of two new superfields $Z_{50}$ and $\bar{Z}_{50}$ in $\mathbf{5 0}$ and $\overline{\mathbf{5 0}}$ representations of SU(5). The decomposition of a $\mathbf{5 0}$ of SU(5) under the SM gauge group does not contain an $\mathrm{SU}(2)$ doublet, but it includes an $\mathrm{SU}(3)$ triplet. Thus, using the 50-plets to generate an effective mass term keeps the electroweak doublets massless, while the colour triplets acquire masses of the order of the GUT scale. The superpotential for the MPM is given by ${ }^{1}$

$$
W_{\mathrm{MPM}}=\bar{H}_{5} H_{75} Z_{50}+\bar{Z}_{50} H_{75} H_{5}+M_{50} Z_{50} \bar{Z}_{50},
$$

where $H_{75}$ is a superfield transforming in the $\mathbf{7 5}$ representation of $\mathrm{SU}(5)$, which contains a SM singlet. When $H_{75}$ gets a vacuum expectation value (VEV) SU(5) is broken to the SM gauge group. ${ }^{2}$ With the triplet mass contribution from $\left\langle H_{75}\right\rangle$ denoted by $V$, the mass matrices of the Higgs fields $H_{5}, \bar{H}_{5}$ and $Z_{50}, \bar{Z}_{50}$ are given by

$$
m_{D}=0, \quad m_{T}=\left(\begin{array}{cc}
0 & V \\
V & M_{50}
\end{array}\right),
$$

for the doublet and triplet components $D$ and $T$ of $H_{5}$ and $Z_{50}$, respectively. The dangerous terms for dimension five proton decay are obtained from the Yukawa couplings

$$
W_{\text {Yuk }}=\mathcal{T}_{i} \mathcal{F}_{j} \bar{H}_{5}+\mathcal{T}_{i} \mathcal{T}_{j} H_{5}
$$

where the families of the MSSM matter superfields are embedded in the standard way in $\mathcal{T}_{i}$ and $\mathcal{F}_{j}$, transforming as $\mathbf{1 0}$ and $\overline{\mathbf{5}}$ of $\mathrm{SU}(5)$, respectively. To calculate the effective dimension five proton decay operators all Higgs triplets from 5- and 50-dimensional representations have to be integrated out, but only the triplets in the 5-dimensional representations dominantly couple to matter. We denote the triplet mass eigenvalues with $\tilde{M}_{1}$ and $\tilde{M}_{2}$, and the corresponding mass eigenstates as $\tilde{T}_{1}$ and $\tilde{T}_{2}$, respectively. The triplets that couple to matter are given by the combinations

$$
T^{(5)}=\sum_{i} U_{1 i}^{*} \tilde{T}_{i}, \quad \bar{T}^{(5)}=\sum_{i} V_{1 i} \overline{\tilde{T}}_{i},
$$

where $U$ and $V$ are unitary matrices defined by $m_{T}=U m_{T}^{\text {diag }} V^{\dagger}$. Integrating out the triplet mass eigenstates $\tilde{T}_{i}$ leads to the effective dimension five operator for proton decay, which is proportional to the inverse of the "effective triplet mass"

$$
\left(M_{T}^{\operatorname{dim}=5}\right)^{-1}:=U_{1 i}^{*}\left(m_{T}^{\mathrm{diag}}\right)_{i j}^{-1} V_{1 j}=U_{1 i}^{*}\left(m_{T}^{\mathrm{diag}}\right)_{i j}^{-1} V_{j 1}^{T}=\left(m_{T}^{-1}\right)_{11} .
$$

Integrating out the heavy colour triplet mass eigenstates also in the Kähler potential

$$
K_{T}=T^{(5)} T^{(5) \dagger}+\bar{T}^{(5)} \bar{T}^{(5) \dagger}+T^{(50)} T^{(50) \dagger}+\bar{T}^{(50)} \bar{T}^{(50) \dagger},
$$

\footnotetext{
${ }^{1}$ For simplicity we omit most order one coefficients in the superpotentials, except where they are relevant to the discussion.

${ }^{2} H_{75}$ can be replaced by the effective combination $H_{24}^{2} / \Lambda$, where $H_{24}$ is the usual GUT-breaking Higgs field in the $\mathbf{2 4}$ representation of $\mathrm{SU}(5)$ [1], see also section 2.5 .
} 
effective dimension six Kähler operators emerge from inserting their equations of motion. The Lagrangian obtained from the D-terms of $K_{T}$ contains baryon number violating four fermion operators. These are proportional to

$$
\left(M_{T}^{\operatorname{dim}=6}\right)^{-2}:=V_{1 i}\left(m_{T}^{\text {diag }}\right)_{i j}^{-1} U_{j k}^{\dagger} U_{k m}\left(m_{T}^{\text {diag }}\right)_{m l}^{-1} V_{l 1}^{\dagger}=\left(m_{T}^{-1} m_{T}^{\dagger-1}\right)_{11}
$$

from $T^{(r)} T^{(r) \dagger}$ and to $\left(M_{\bar{T}}^{\operatorname{dim}=6}\right)^{-2}=\left(m_{T}^{\dagger-1} m_{T}^{-1}\right)_{11}$ from $\bar{T}^{(r)} \bar{T}^{(r) \dagger}$. With the mass matrix $m_{T}$ given in eq. (2.2), the effective triplet mass is thus ${ }^{3}$

$$
M_{T}^{\text {dim }=5}=\left(m_{T}^{-1}\right)_{11}^{-1}=-\frac{V^{2}}{M_{50}},
$$

while the suppression of dimension six proton decay is given by

$$
\left(M_{T}^{\operatorname{dim}=6}\right)^{2}=\left(M_{\bar{T}}^{\operatorname{dim}=6}\right)^{2}=\left(m_{T}^{-1} m_{T}^{\dagger-1}\right)_{11}^{-1}=\frac{|V|^{4}}{\left|M_{50}\right|^{2}+|V|^{2}} .
$$

Note that with a GUT scale value of $V \approx 10^{16} \mathrm{GeV}$ and $M_{50}$ below the Planck scale, the dimension six proton decay is suppressed sufficiently with values of $M_{T}^{\mathrm{dim}=6}$ between $10^{13}$ and $10^{16} \mathrm{GeV}$. Since the doublets obtain no mass terms, the splitting of doublet mass and effective triplet mass is achieved. Using $M_{T}^{\text {dim }=5} \gtrsim 10^{17} \mathrm{GeV}$ one obtains an upper bound for $M_{50} \lesssim 10^{15} \mathrm{GeV}$. Having the large representations $\mathbf{5 0}$ and $\overline{\mathbf{5 0}}$ enter the Renormalization Group Equations (RGEs) at this low mass scale, however, leads to the break down of perturbativity just above the GUT scale. Thus, the MPM solves the DTS problem — but trades it for $\mathrm{SU}(5)$ becoming non-perturbative much below the Planck scale $M_{\mathrm{Pl}}$.

\subsection{The double missing partner mechanism}

This trade-off can be avoided in the double missing partner mechanism (DMPM), where the number of Higgs fields in $\mathbf{5}, \overline{\mathbf{5}}, \mathbf{5 0}$ and $\overline{\mathbf{5 0}}$ representations gets doubled [9]. The fields $H_{5}$ and $\bar{H}_{5}$ couple to the matter fields $\mathcal{F}_{i}$ and $\mathcal{T}_{i}$, whereas $H_{5}^{\prime}$ and $\bar{H}_{5}^{\prime}$ do not. The superpotential for the DMPM is given by

$$
\begin{aligned}
W_{\mathrm{DMPM}}= & \bar{H}_{5} H_{75} Z_{50}+\bar{Z}_{50} H_{75} H_{5}^{\prime}+\bar{H}_{5}^{\prime} H_{75} Z_{50}^{\prime}+\bar{Z}_{50}^{\prime} H_{75} H_{5} \\
& +M_{50} Z_{50} \bar{Z}_{50}+M_{50}^{\prime} Z_{50}^{\prime} \bar{Z}_{50}^{\prime} \\
& +\mu^{\prime} H_{5}^{\prime} \bar{H}_{5}^{\prime} .
\end{aligned}
$$

The mass matrices of the doublet and triplet components of the Higgs fields $H_{5}, H_{5}^{\prime}, Z_{50}$, $Z_{50}^{\prime}$ and their corresponding barred fields after $H_{75}$ gets a VEV $V$ are given by

$$
m_{D}=\left(\begin{array}{cc}
0 & 0 \\
0 & \mu^{\prime}
\end{array}\right), \quad m_{T}=\left(\begin{array}{cccc}
0 & 0 & 0 & V \\
0 & \mu^{\prime} & V & 0 \\
V & 0 & M_{50} & 0 \\
0 & V & 0 & M_{50}^{\prime}
\end{array}\right) .
$$

\footnotetext{
${ }^{3}$ In the text when we quote numbers for $M_{T}^{\mathrm{dim}=5}, M_{T}^{\mathrm{dim}=6}$ and $M_{\bar{T}}^{\mathrm{dim}=6}$ we will always refer to their absolute values.
} 
While the Higgs doublets coupling to matter remain massless, the second pair of Higgs doublets contained in $H_{5}^{\prime}$ and $\bar{H}_{5}^{\prime}$ has mass $\mu^{\prime}$. The improvement of the DMPM compared to the MPM can be seen from the effective triplet mass $M_{T}^{\operatorname{dim}=5}$

$$
M_{T}^{\operatorname{dim}=5}=\left(m_{T}^{-1}\right)_{11}^{-1}=-\frac{V^{4}}{\mu^{\prime} M_{50} M_{50}^{\prime}} .
$$

The same effective triplet mass of $M_{T}^{\operatorname{dim}=5} \approx 10^{17} \mathrm{GeV}$ can now be obtained while keeping high masses $M_{50} \approx M_{50}^{\prime} \approx 10^{18} \mathrm{GeV}$, provided the heavier doublet pair has a (relatively) small mass $\mu^{\prime} \approx 10^{11} \mathrm{GeV}$. With the large representations of SU(5) having high masses, the perturbativity of the model can be preserved up to (almost) the Planck scale. Dimension six proton decay is suppressed by

$$
\begin{aligned}
& \left(M_{T}^{\operatorname{dim}=6}\right)^{2}=\left(m_{T}^{-1} m_{T}^{\dagger-1}\right)_{11}^{-1}=\frac{|V|^{8}}{|V|^{6}+\left|M_{50}\right|^{2}\left(|V|^{4}+\left|M_{50}^{\prime} \mu^{\prime}\right|^{2}+\left|V \mu^{\prime}\right|^{2}\right)} \approx\left(10^{14} \mathrm{GeV}\right)^{2} \\
& \left(M_{\bar{T}}^{\operatorname{dim}=6}\right)^{2}=\left(m_{T}^{\dagger-1} m_{T}^{-1}\right)_{11}^{-1}=\frac{|V|^{8}}{|V|^{6}+\left|M_{50}^{\prime}\right|^{2}\left(|V|^{4}+\left|M_{50} \mu^{\prime}\right|^{2}+\left|V \mu^{\prime}\right|^{2}\right)} \approx\left(10^{14} \mathrm{GeV}\right)^{2},
\end{aligned}
$$

in agreement with the bounds on proton decay.

\subsection{Planck-scale suppressed operators}

The philosophy we follow in this paper is to consider all Planck-scale suppressed operators allowed by the symmetries.

The superpotentials in eq. (2.1) and eq. (2.10) include mass terms for the 50-dimensional messengers. As such, one cannot use symmetries to forbid non-renormalizable Planckscale suppressed operators such as $H_{5} H_{75}^{2} \bar{H}_{5} / M_{\mathrm{Pl}}$ (for the MPM, and $H_{5} H_{75}^{2} \bar{H}_{5}^{\prime} / M_{\mathrm{Pl}}$ and $H_{5}^{\prime} H_{75}^{2} \bar{H}_{5} / M_{\mathrm{Pl}}$ for the DMPM). These Planck-scale suppressed operators do not involve the 50-dimensional messengers and therefore generate dangerously large contributions to the masses of the doublets contained in the 5-dimensional representations, effectively spoiling the mechanism.

Given our philosophy, we must forbid these operators through a shaping symmetry. The MPM and the DMPM can then be restored by adding a singlet field $S$, responsible for giving mass to the 50-dimensional superfields through couplings of the form $S Z_{50} \bar{Z}_{50}$, $S Z_{50}^{\prime} \bar{Z}_{50}^{\prime}$ and a VEV $\langle S\rangle \neq 0$, as seen in the diagram in figure 1 (note $S$ is not acting as an external field but generating the mass term). The non-trivial charge of $S$ under a shaping symmetry forbids the dangerous Planck-suppressed operators.

We will generalise this strategy of generating masses for the messenger fields through an additional singlet field in sections 2.5 and 4, to avoid similarly Planck-scale suppressed operators spoiling the DMPM or the predictions for the Yukawa coupling ratios.

\subsection{Yukawa coupling ratios}

The problem of non-viable GUT predictions for the fermion masses in the minimal SU(5) model, such as $Y_{e}=Y_{d}^{T}$, can be solved through effective Yukawa couplings generated from 
$\langle S\rangle$

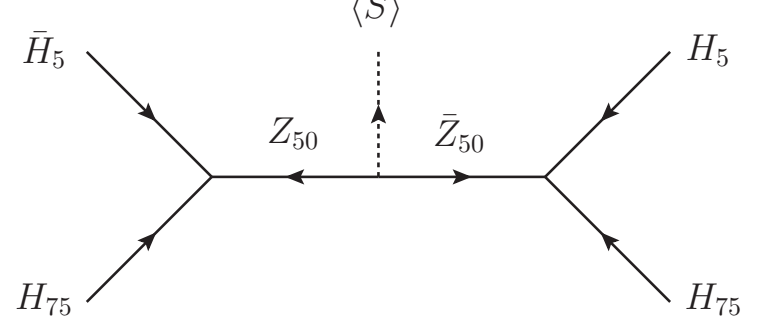

Figure 1. MPM diagram with an external $S$ field generating the mass term for the 50-dimensional messengers after getting a VEV.

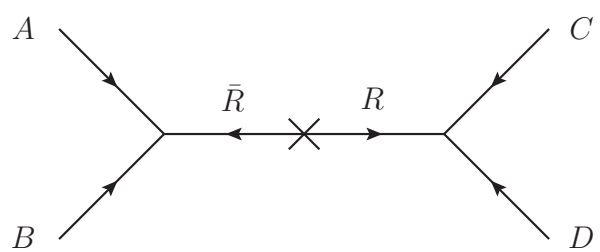

Figure 2. Supergraphs generating effectively Yukawa couplings upon integrating out the pair of messengers fields $R$ and $\bar{R}$.

\begin{tabular}{|cccc|}
\hline$A B$ & $C D$ & $R$ & $\left(Y_{e}\right)_{j i} /\left(Y_{d}\right)_{i j}$ \\
\hline$H_{24} \mathcal{F}$ & $\mathcal{T} \bar{H}_{45}$ & $\overline{\mathbf{4 5}}$ & $-\frac{1}{2}$ \\
$H_{24} \mathcal{F}$ & $\mathcal{T} \bar{H}_{5}$ & $\overline{\mathbf{5}}$ & $-\frac{3}{2}$ \\
$H_{24} \mathcal{T}$ & $\mathcal{F} \bar{H}_{5}$ & $\mathbf{1 0}$ & 6 \\
\hline
\end{tabular}

Table 1. CG factors for the dimension five effective operators $W \supset(A B)_{R}(C D)_{\bar{R}}$. See the main text, figure 2 and $[11,22]$ for more details.

higher dimensional operators. When the higher dimensional operators contain a GUT breaking Higgs field, new ratios between the Yukawa couplings of down-type quarks and charged leptons can emerge once the GUT symmetry gets spontaneously broken [11].

Due to the introduction of extra SU(5) non-singlet fields, which participate in higher dimensional operators, there are in general several ways to construct invariants, namely multiple ways to contract the $\mathrm{SU}(5)$ indices. In the general case, such effective operators are not predictive and introduce an arbitrary, linear combination of several CG factors.

This issue is generic in flavour models and can be resolved by constructing a specific UV completion of the effective operators, see $[20,21]$ for mixing in lepton models, $[11,22]$ for GUT relations, and for applications in (GUT) flavour models, e.g. [15-17]. By introducing pairs of heavy messengers in the $\mathrm{SU}(5)$ representations $\mathbf{R}$ and $\overline{\mathbf{R}}$, the renormalizable couplings associated with the effective operators are specified and a unique contraction of SU(5) indices of the effective operator is obtained simply by integrating out the messenger fields.

In figure 2 and table 1 , we briefly review the topology of the diagram and field combinations that lead to the CG factors $\left(Y_{e}\right)_{j i} /\left(Y_{d}\right)_{i j}=-\frac{1}{2},-\frac{3}{2}$ and 6 for SU(5) GUTs, which have been shown to be useful for flavour model building [11-13]. To generate these relations we use a GUT breaking Higgs field $H_{24}$ in the adjoint representation of SU(5), and Higgs fields $\bar{H}_{5}$ and $\bar{H}_{45}$ in the $\overline{\mathbf{5}}$ and $\overline{\mathbf{4 5}}$ representation. The factor $-\frac{1}{2}$ is, for instance, generated by the coupling of $H_{24}$ and $\mathcal{F}$ to a messenger field transforming as $\mathbf{4 5}$, while its partner $\overline{\mathbf{4 5}}$ couples to $\mathcal{T}$ and $\bar{H}_{45}$. In our models in section 4 the same CG factor $-\frac{1}{2}$ is obtained from a dimension six operator where $\bar{H}_{45}$ acts as heavy messenger field coupling to $H_{24}$ and $\bar{H}_{5}$. 
$\langle S\rangle$

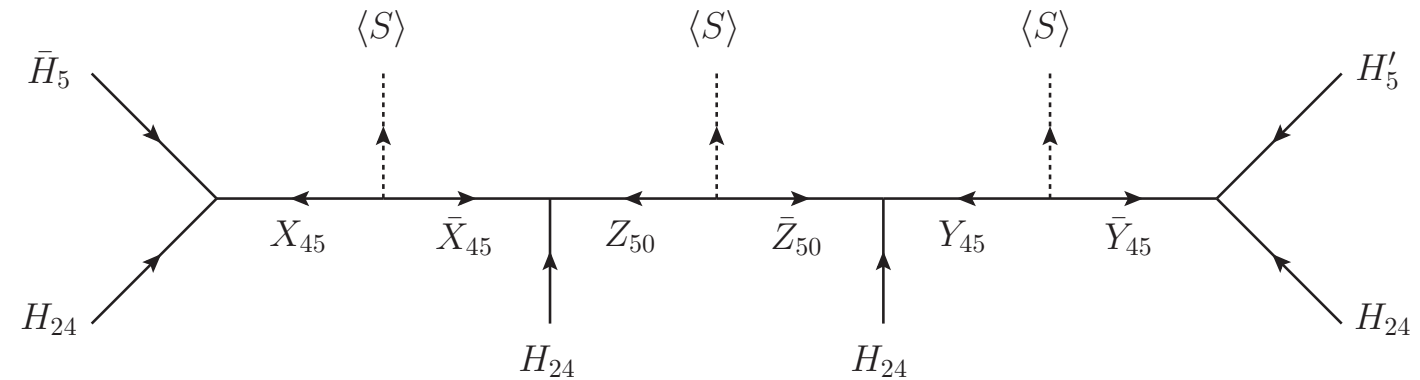

$\langle S\rangle$

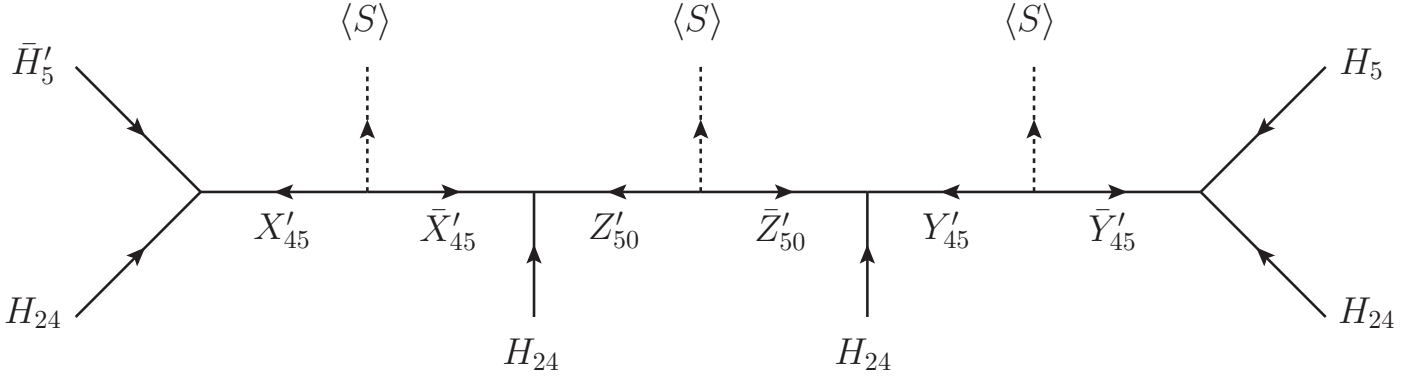

Figure 3. Supergraphs generating the non-diagonal entries of the triplet mass matrix.

So far we have discussed CG factors between the MSSM Yukawa couplings. For an analysis of proton decay, the CG factors to the Yukawa couplings of the colour Higgs triplets also play an important role. In appendix A we present an extensive discussion of CG factors in $\mathrm{SU}(5)$, including those.

\subsection{The double missing partner mechanism with an adjoint}

As we discussed in the previous subsection, the CG factors we want to combine with the DMPM require the GUT breaking Higgs field to be in the adjoint representation of $\mathrm{SU}(5), 24$. This motivates us to replace the Higgs field $H_{75}$ needed for the DMPM with the effective combination $H_{24}^{2} / \Lambda$ [1], which at the renormalizable level can be obtained by integrating out heavy messenger fields in the $\mathbf{4 5}$ and $\overline{\mathbf{4 5}}$ representations of SU(5) [5]. To replace the $H_{75}$ in the MPM, we have to introduce a set of messenger fields $X_{45}, \bar{X}_{45}, Y_{45}$ and $\bar{Y}_{45}$. For the DMPM, we also need to add a second set $X_{45}^{\prime}, \bar{X}_{45}^{\prime}, Y_{45}^{\prime}$ and $\bar{Y}_{45}^{\prime}$. In figure 3 we show the supergraphs generating the non-diagonal entries of the triplet mass matrix in the DMPM with an adjoint $H_{24}$.

In analogy with the discussion in section 2.3, we avoid direct mass terms in order to forbid dangerous Planck-suppressed operators that would generate universal mass contributions for Higgs doublets and triplets. The messenger pairs $X_{45} \bar{X}_{45}, Y_{45} \bar{Y}_{45}, Z_{50} \bar{Z}_{50}$ and their corresponding primed versions obtain masses from the VEV of a singlet field $S$, charged under an additional shaping symmetry. We specify these symmetries for two example models in section 4.

One may wonder if some of these heavy 45-dimensional messengers could be the same, so that the number of fields in the spectrum would be reduced while preserving the structure of the mechanism. But if either $X_{45} \equiv Y_{45}$ or $X_{45}^{\prime} \equiv Y_{45}^{\prime}$, it can be seen from figure 3 that 
supergraphs without the $Z_{50} \bar{Z}_{50}$ mass insertion would be allowed, spoiling the splitting of doublets and triplets and generating large non-diagonal entries in $m_{D}$. In turn, if either $X_{45} \equiv X_{45}^{\prime}, Y_{45} \equiv Y_{45}^{\prime}$ or $Z_{50} \equiv Z_{50}^{\prime}$, the DMPM is reduced to the MPM, reintroducing the issue of perturbativity. Finally, an identification of $X_{45} \equiv Y_{45}^{\prime}$ would allow diagrams bypassing the 50-dimensional fields, generating unwanted mass term for both doublet and triplet components of $H_{5}, \bar{H}_{5}$, and thus a too large $\mu$-term.

With this messenger superfield content, we carefully checked that no dangerous Plancksuppressed operators spoil the mechanism. The renormalizable superpotential is:

$$
\begin{aligned}
W_{\mathrm{DMPM} 24}= & \bar{H}_{5} H_{24} X_{45}+\bar{X}_{45} H_{24} Z_{50}+\bar{Z}_{50} H_{24} Y_{45}+\bar{Y}_{45} H_{24} H_{5}^{\prime} \\
& +\bar{H}_{5}^{\prime} H_{24} X_{45}^{\prime}+\bar{X}_{45}^{\prime} H_{24} Z_{50}^{\prime}+\bar{Z}_{50}^{\prime} H_{24} Y_{45}^{\prime}+\bar{Y}_{45}^{\prime} H_{24} H_{5} \\
& +S X_{45} \bar{X}_{45}+S Y_{45} \bar{Y}_{45}+S Z_{50} \bar{Z}_{50}+S X_{45}^{\prime} \bar{X}_{45}^{\prime}+S Y_{45}^{\prime} \bar{Y}_{45}^{\prime}+S Z_{50}^{\prime} \bar{Z}_{50}^{\prime} \\
& +\mu^{\prime} H_{5}^{\prime} \bar{H}_{5}^{\prime} .
\end{aligned}
$$

After $H_{24}$ and $S$ obtain their VEVs and integrating out the 45-dimensional messenger fields, we find the mass matrices for the Higgs doublets and triplets to be

$$
m_{D}=\left(\begin{array}{cc}
0 & 0 \\
0 & \mu^{\prime}
\end{array}\right), \quad m_{T}=\left(\begin{array}{cccc}
0 & 0 & 0 & -\frac{V^{2}}{\langle S\rangle} \\
0 & \mu^{\prime} & -\frac{V^{2}}{\langle S\rangle} & 0 \\
-\frac{V^{2}}{\langle S\rangle} & 0 & \langle S\rangle & 0 \\
0 & -\frac{V^{2}}{\langle S\rangle} & 0 & \langle S\rangle
\end{array}\right),
$$

which one can easily compare to the ones of eq. (2.11) in section 2.5. Here $V$ is defined by $\left\langle H_{24}\right\rangle \equiv V \operatorname{diag}\left(1,1,1,-\frac{3}{2},-\frac{3}{2}\right)$. Integrating out the heavy 50-dimensional fields in a next step, the mass matrices become

$$
m_{D}=\left(\begin{array}{cc}
0 & 0 \\
0 & \mu^{\prime}
\end{array}\right), \quad m_{T}=\left(\begin{array}{cc}
0 & -\frac{V^{4}}{\langle S\rangle^{3}} \\
-\frac{V^{4}}{\langle S\rangle^{3}} & \mu^{\prime}
\end{array}\right) .
$$

Thus, the doublets in the pair $H_{5} \bar{H}_{5}$ stay massless, while the doublet pair in $H_{5}^{\prime} \bar{H}_{5}^{\prime}$ is heavy. Using only $H_{5}$ and $\bar{H}_{5}$ for the Yukawa couplings of the SM fermions, the effective triplet component mass relevant for dimension 5 proton decay is given by

$$
M_{T}^{\operatorname{dim}=5}=\left(m_{T}^{-1}\right)_{11}^{-1}=-\frac{V^{8}}{\langle S\rangle^{6} \mu^{\prime}} .
$$

The effective masses suppressing dimension six proton decay mediated by colour triplets are given by

$$
\left(M_{T}^{\operatorname{dim}=6}\right)^{2}=\left(M_{\bar{T}}^{\operatorname{dim}=6}\right)^{2} \approx \frac{|V|^{8}}{|\langle S\rangle|^{6}},
$$

where we used the fact that $|\langle S\rangle| \gg|V|$ and $\left|\langle S\rangle^{3} \mu^{\prime}\right| \ll|V|^{4}$. Now the requirement of $M_{T}^{\operatorname{dim}=6} \gtrsim 10^{12} \mathrm{GeV}$ can only be obtained with $\langle S\rangle \approx 10^{18} \mathrm{GeV}$ if the GUT scale value is larger than $V \gtrsim 10^{16} \mathrm{GeV}$. In this case one needs $\mu^{\prime} \approx 10^{7} \mathrm{GeV}$ to obtain an effective triplet mass $M_{T}^{\operatorname{dim}=5} \approx 10^{17} \mathrm{GeV}$ for dimension five proton decay operators. Therefore, if 
the GUT scale is high enough, the effective triplet mass can be large enough to stabilize the proton, while the large $\mathrm{SU}(5)$ representations used in the DMPM can be heavy enough to keep the theory perturbative up to the Planck scale.

When $H_{24}$ is uncharged under additional symmetries, having $\mu^{\prime}$ several orders of magnitude smaller than $V$ requires $\mu^{\prime}$ to arise from the spontaneous breakdown of a shaping symmetry, to avoid the term $\left\langle H_{24}\right\rangle H_{5}^{\prime} \bar{H}_{5}^{\prime}$, which would give rise to a much too small effective triplet mass. Note that the effective triplet masses entering dimension five proton decay can be expressed in terms of the mass eigenstates of doublet and triplet components as $M_{T}^{\operatorname{dim}=5}=-\frac{\tilde{M}_{1} \tilde{M}_{2}}{\mu^{\prime}}$. The effective triplet mass of dimension six proton decay is then excellently approximated by $M_{\bar{T}}^{\operatorname{dim}=6}=M_{T}^{\operatorname{dim}=6} \approx \sqrt{M_{T}^{\operatorname{dim}=5} \mu^{\prime}}$.

\subsection{Introducing a second adjoint field}

We have seen that the DMPM with an adjoint GUT breaking Higgs instead of a $\mathbf{7 5}$ already solves the DTS problem while providing the necessary building block for the desirable CG factors for flavour model building, if the GUT scale is high enough. To conclude this section, we will argue why it is compelling to further introduce a second adjoint Higgs field:

- In the minimal SUSY SU(5) model [23], the single GUT breaking 24 contains an $\mathrm{SU}(2)$ triplet component and an SU(3) octet component with equal masses. Demanding gauge coupling unification, the mass of the Higgs colour triplets is required to be about $10^{15} \mathrm{GeV}$ [6], ruling out this model due to proton decay. Non-renormalizable operators in the GUT breaking superpotential can split the $\mathbf{2 4}$ component masses, allowing a higher effective triplet mass [24] (see also section 3). An additional 24 can be used to realize this non-renormalizable superpotential in a renormalizable way.

- It turns out that the introduction of an additional $\mathbf{2 4}$ is not just a UV-completion of the non-renormalizable superpotential of [24]. When both adjoints have approximately the same mass and therefore the second $\mathbf{2 4}$ is not integrated out, the additional colour octet and electroweak triplet in the spectrum lead to more freedom for the GUT scale and effective triplet mass. In the following section we discuss all possible renormalizable superpotentials with two adjoints and their impact on $M_{\mathrm{GUT}}$ and $M_{T}^{\text {dim=5 }}$ from a gauge coupling unification analysis.

- A renormalizable superpotential for one $\mathbf{2 4}$ requires it to be uncharged under shaping symmetries in order for it to obtain a VEV. However, such a shaping symmetry charge is vital in the type of flavour models considered here, to avoid unwanted admixtures of additional CG factors involving less insertions of $H_{24}$. With a second $\mathbf{2 4}$, the adjoint fields can acquire non-vanishing VEVs even when charged under shaping symmetries.

These features of renormalizable superpotentials for two adjoints are presented in detail in the next section. 


\section{Grand unification and the effective triplet mass}

In GUT extensions of the SM it is quite common to have additional fields below the GUT scale that modify the RGE running, as it is the case for the class of models in this paper. Therefore one has to study the impact of the additional fields on the running of the gauge couplings and especially study their unification. The modified unification condition for the gauge couplings at one-loop reads

$$
\frac{1}{\alpha_{u}}=\frac{1}{\alpha_{i}}-\frac{1}{2 \pi}\left(b_{i}^{(\mathrm{SM})} \log \frac{M_{\mathrm{SUSY}}}{M_{Z}}+b_{i}^{(\mathrm{MSSM})} \log \frac{M_{\mathrm{GUT}}}{M_{\mathrm{SUSY}}}+\sum_{f} b_{i}^{(f)} \log \frac{M_{\mathrm{GUT}}}{M_{f}}\right),
$$

where $i=1,2,3$ labels the SM gauge interaction and $f$ labels the additional superfields (compared to the MSSM), with masses $M_{f}$ and $\beta$ coefficients $b_{i}^{(f)}$. The one-loop $\beta$-function coefficients for the SM are $b_{i}^{(\mathrm{SM})}=(41 / 10,-19 / 6,-7)$ and for the MSSM $b_{i}^{(\mathrm{MSSM})}=$ $(33 / 5,1,-3)$. The SUSY scale $M_{\mathrm{SUSY}}$ is defined here as the scale where we make the transition from the SM $\beta$ coefficients to the MSSM ones. The $\alpha_{i}$ are defined at low energies $\alpha_{i} \equiv \alpha_{i}\left(M_{Z}\right)$ while $\alpha_{u}$ is the unified gauge coupling at the GUT scale $\alpha_{u} \equiv \alpha_{i}\left(M_{\mathrm{GUT}}\right)$. The GUT scale $M_{\mathrm{GUT}}$ is defined here as the scale where the last $\mathrm{SU}(5)$ multiplet is completed, in other words the scale where all three one-loop $\beta$ coefficients for the SM gauge couplings become equal.

From now on, we assume that the heaviest incomplete SU(5) multiplets to enter the RGE running are the leptoquark vector bosons, such that the GUT scale corresponds to their mass $M_{\mathrm{GUT}}=M_{V}$. While other cases can certainly arise, we focus on this option because it is quite common in our setup to have heavy leptoquark vector bosons, and furthermore we verified that in this case the triplet mass can be made very heavy as well.

In addition to the MSSM field content, the DMPM introduces one additional pair of $\mathrm{SU}(2)$-doublets, $D^{(5)}$ and $\bar{D}^{(5)}$ and two additional pairs of SU(3)-triplets, $T_{i}^{(5)}$ and $\bar{T}_{i}^{(5)}$, $i=1,2$. They enter the $\beta$-functions with the coefficients $b_{i}^{(5, D)}=(3 / 5,1,0)$ for the Dirac pair of doublets and $b_{i}^{(5, T)}=(2 / 5,0,1)$ per Dirac pair of triplet and anti-triplet.

Furthermore, we use two SU(5) breaking GUT Higgs fields $H_{24}$ and $H_{24}^{\prime}$ in the adjoint representation. They contain one SM-singlet component each, one SU(2)-triplet, $T^{(24)}$, with $b_{i}^{(24, T)}=(0,2,0)$, an $\mathrm{SU}(3)$-octet, $O^{(24)}$ with $b_{i}^{(24, O)}=(0,0,3)$ and a leptoquark superfield pair, $L^{(24)}$ with $b_{i}^{(24, L)}=(5,3,2)$. Since one leptoquark superfield pair is eaten up during the breaking of $\mathrm{SU}(5)$, we are left with two triplets with masses $M_{T_{1}^{(24)}}$ and $M_{T_{2}^{(24)}}$, two octets with masses $M_{O_{1}^{(24)}}$ and $M_{O_{2}^{(24)}}$ and one leptoquark superfield pair with mass $M_{L^{(24)}}$.

For convenience we define the geometric means of the masses $M_{T^{(5)}}^{2}=M_{T_{1}^{(5)}} M_{T_{2}^{(5)}}$ for the colour triplets and analogously $M_{T^{(24)}}^{2}=M_{T_{1}^{(24)}} M_{T_{2}^{(24)}}, M_{O^{(24)}}^{2}=M_{O_{1}^{(24)}} M_{O_{2}^{(24)}}$ for the components of $H_{24}$ and $H_{24}^{\prime}$. 
Having this at hand, we can solve eq. (3.1) for $M_{D^{(5)}}, M_{T^{(5)}}$ and $M_{\mathrm{GUT}},{ }^{4}$

$$
\begin{aligned}
\log M_{D^{(5)}}= & \frac{15 \pi}{4 \alpha_{1}}-\frac{17 \pi}{4 \alpha_{2}}-\frac{3 \pi}{2 \alpha_{3}}+\frac{59}{3} \log M_{Z} \\
& +\frac{2 \pi}{\alpha_{u}}+\frac{3}{2} \log M_{L^{(24)}}-\frac{17}{2} \log M_{T^{(24)}}-\frac{9}{2} \log M_{O^{(24)}}-\frac{43}{6} \log M_{\mathrm{SUSY}}, \\
\log M_{T^{(5)}}= & \frac{35 \pi}{24 \alpha_{1}}-\frac{7 \pi}{8 \alpha_{2}}-\frac{19 \pi}{12 \alpha_{3}}+\frac{119}{12} \log M_{Z} \\
& +\frac{\pi}{\alpha_{u}}+\frac{3}{4} \log M_{L^{(24)}}-\frac{7}{4} \log M_{T^{(24)}}-\frac{19}{4} \log M_{O^{(24)}}-\frac{19}{6} \log M_{\mathrm{SUSY}}, \\
\log M_{\mathrm{GUT}}= & \frac{5 \pi}{12 \alpha_{1}}-\frac{\pi}{4 \alpha_{2}}-\frac{\pi}{6 \alpha_{3}}+\frac{11}{6} \log M_{Z} \\
& +\frac{1}{2} \log M_{L^{(24)}}-\frac{1}{2} \log M_{T^{(24)}}-\frac{1}{2} \log M_{O^{(24)}}-\frac{1}{3} \log M_{\mathrm{SUSY}} .
\end{aligned}
$$

For the study of proton decay, it is more convenient to instead solve eq. (3.1) for the GUT scale gauge coupling $\alpha_{u}$ and the effective triplet mass $M_{T}^{\mathrm{dim}=5}=M_{T^{(5)}}^{2} / M_{D^{(5)}}$, which gives the suppression of the dimension 5 proton decay operators (cf. the discussion in section 2.5). Then we get the relations

$$
\begin{aligned}
\frac{\pi}{\alpha_{u}}= & -\frac{43 \pi}{24 \alpha_{1}}+\frac{15 \pi}{8 \alpha_{2}}+\frac{11 \pi}{12 \alpha_{3}}-\frac{197}{20} \log M_{Z}+\frac{3}{5} \log M_{D T} \\
& -\frac{3}{4} \log M_{L^{(24)}}+\frac{15}{4} \log M_{T^{(24)}}+\frac{11}{4} \log M_{O^{(24)}}+\frac{7}{2} \log M_{\mathrm{SUSY}}, \\
\log M_{T}^{\mathrm{dim}=5}= & -\frac{5 \pi}{6 \alpha_{1}}+\frac{5 \pi}{2 \alpha_{2}}-\frac{5 \pi}{3 \alpha_{3}}+\frac{1}{6} \log M_{Z} \\
& +5 \log M_{T^{(24)}}-5 \log M_{O^{(24)}}+\frac{5}{6} \log M_{\mathrm{SUSY}}, \\
\log M_{\mathrm{GUT}}= & \frac{5 \pi}{12 \alpha_{1}}-\frac{\pi}{4 \alpha_{2}}-\frac{\pi}{6 \alpha_{3}}+\frac{11}{6} \log M_{Z} \\
& +\frac{1}{2} \log M_{L^{(24)}}-\frac{1}{2} \log M_{T^{(24)}}-\frac{1}{2} \log M_{O^{(24)}}-\frac{1}{3} \log M_{\mathrm{SUSY}},
\end{aligned}
$$

where we have introduced the mass $M_{D T}^{3}=M_{D^{(5)}}^{2} M_{T^{(5)}}$. As one can see only $\alpha_{u}$ depends on $M_{D T}$, which is due to the fact that doublets and colour triplets together form a complete representation of $\mathrm{SU}(5)$. Thus, following eq. (3.1), one can see that a simultaneous rescaling $M_{D^{(5)}} \rightarrow q^{2} M_{D^{(5)}}$ and $M_{T^{(5)}} \rightarrow q M_{T^{(5)}}$ leaves the GUT scale invariant and only shifts $\alpha_{u}$, while $M_{T}^{\text {dim=5 }} \propto q^{0}$ remains unchanged and $M_{D T} \propto q$ parametrises this rescaling. Further interdependencies between $\alpha_{u}, M_{T}^{\operatorname{dim}=5}$ and $M_{\mathrm{GUT}}$ are then implicit via their shared dependence on the other masses.

Thus, unification implies that the effective triplet mass follows the relation

$$
\begin{aligned}
M_{T}^{\mathrm{dim}=5} & =\exp \left(\frac{5}{6} \pi\left(\frac{3}{\alpha_{2}}-\frac{2}{\alpha_{3}}-\frac{1}{\alpha_{1}}\right)\right) M_{Z}^{1 / 6} M_{\mathrm{SUSY}}^{\frac{5}{6}}\left(\frac{M_{T^{(24)}}}{M_{O^{(24)}}}\right)^{5} \\
& =2.5_{-0.8}^{+0.6} \cdot 10^{17} \mathrm{GeV}\left(\frac{M_{\mathrm{SUSY}}}{1 \mathrm{TeV}}\right)^{\frac{5}{6}}\left(\frac{M_{T^{(24)}}}{M_{O^{(24)}}}\right)^{5}
\end{aligned}
$$

\footnotetext{
${ }^{4}$ In the following, "log" of a mass is to be understood as the natural logarithm of the mass divided by one common mass scale, e.g. $\log m \equiv \log (m / \mathrm{GeV})$.
} 
while the GUT scale is given by

$$
M_{\mathrm{GUT}}=1.37_{-0.05}^{+0.05} \cdot 10^{16} \mathrm{GeV}\left(\frac{M_{\mathrm{SUSY}}}{1 \mathrm{TeV}}\right)^{-\frac{1}{3}}\left(\frac{M_{L^{(24)}}}{10^{16} \mathrm{GeV}}\right)^{\frac{1}{2}}\left(\frac{M_{T^{(24)}} M_{O^{(24)}}}{\left(10^{16} \mathrm{GeV}\right)^{2}}\right)^{-\frac{1}{2}} .
$$

For completeness, the unified gauge coupling is given by

$$
\begin{aligned}
\frac{1}{\alpha_{u}}= & 24.58 \pm 0.06+\frac{7}{2 \pi} \ln \frac{M_{\mathrm{SUSY}}}{1 \mathrm{TeV}}+\frac{3}{5 \pi} \ln \frac{M_{D T}}{10^{14} \mathrm{GeV}} \\
& -\frac{3}{4 \pi} \ln \frac{M_{L^{(24)}}}{10^{16} \mathrm{GeV}}+\frac{15}{4 \pi} \ln \frac{M_{T^{(24)}}}{10^{16} \mathrm{GeV}}+\frac{11}{4 \pi} \ln \frac{M_{O^{(24)}}}{10^{16} \mathrm{GeV}} .
\end{aligned}
$$

For these numbers, we have used the experimental values and uncertainties for the gauge couplings found in [10]. Note that for all three quantities the resulting uncertainty is dominated by the experimental error on $\alpha_{s}$. In the following we will not quote any errors on the masses anymore since the relative uncertainty changes only negligibly for the different superpotentials and for two-loop running. The reference scale $10^{14} \mathrm{GeV}$ is chosen due to the fact that $M_{D T}=10^{14} \mathrm{GeV}$ and $M_{T^{(5)}}=10^{16} \mathrm{GeV}$ implies $M_{T}^{\text {dim=5 }}=10^{19} \mathrm{GeV}$.

Since the effective triplet mass $M_{T}^{\operatorname{dim}=5}$ receives significant two-loop contributions (cf., for instance, [6]), we have also implemented a numerical two-loop RGE analysis using the following procedure. We start with SM values for the gauge and Yukawa couplings [25] at $M_{Z}$, run up to to a scale of $1 \mathrm{TeV}$ with the full two-loop SM RGEs and match the SM to the MSSM (including $\overline{\mathrm{MS}}$ to $\overline{\mathrm{DR}}$ scheme conversion). From there we run and match using full two-loop MSSM RGEs and one-loop gauge coupling threshold corrections ${ }^{5}$ while step-by-step including all additional multiplets at their mass scale via their contributions to the one- and two-loop gauge coupling RGEs, see appendix B. The Yukawa couplings of the Higgs colour triplets are well approximated by using the Yukawa couplings of the corresponding doublets. We do not take into account any other Yukawa couplings. We verified this approximation numerically and the results for the colour triplet masses are barely affected.

\subsection{Superpotentials with two adjoints of SU(5)}

In this section, we systematically study all superpotentials with two adjoints that can break $\mathrm{SU}(5)$ to the SM gauge group. We find only four possibilities with non-vanishing VEVs and masses. Classified based on their symmetry, they are:

(a) $W=M_{24} \operatorname{tr} H_{24}^{2}+M_{24}^{\prime} \operatorname{tr} H_{24}^{\prime 2}+\kappa^{\prime} \operatorname{tr} H_{24} H_{24}^{\prime 2}+\lambda \operatorname{tr} H_{24}^{3}$,

$\mathbb{Z}_{2}$ symmetry where $H_{24}$ is uncharged and $H_{24}^{\prime}$ charged.

(b) $W=\tilde{M}_{24} \operatorname{tr} H_{24} H_{24}^{\prime}+\lambda \operatorname{tr} H_{24}^{3}+\lambda^{\prime} \operatorname{tr} H_{24}^{\prime 3}$,

$\mathbb{Z}_{3}$ symmetry, where $H_{24}$ has charge 2 and $H_{24}^{\prime}$ charge 1 .

(c) $W=\tilde{M}_{24} \operatorname{tr} H_{24} H_{24}^{\prime}+\lambda \operatorname{tr} H_{24}^{3}+\kappa^{\prime} \operatorname{tr} H_{24} H_{24}^{\prime 2}$, $\mathbb{Z}_{4}^{R}$ symmetry where $H_{24}$ has a charge of 2 (with $q_{\theta}=1$ ) and $H_{24}^{\prime}$ is uncharged.

\footnotetext{
${ }^{5}$ When one integrates out particles at a threshold scale equal to their mass, these threshold corrections vanish, as can be seen in [26].
} 
(d) The trivial case with both fields only charged under SU(5) and all (non-linear) terms allowed. We will not consider this case any further.

Since we are dealing with two adjoint Higgs fields, it is convenient to define a quantity $\tan \beta_{V}$ similar to $\tan \beta$ of the MSSM, so that

$$
\begin{aligned}
& \left\langle H_{24}\right\rangle=V_{1} \mathrm{e}^{\mathrm{i} \phi_{1}} \operatorname{diag}(1,1,1,-3 / 2,-3 / 2), \\
& \left\langle H_{24}^{\prime}\right\rangle=V_{2} \mathrm{e}^{\mathrm{i} \phi_{2}} \operatorname{diag}(1,1,1,-3 / 2,-3 / 2),
\end{aligned}
$$

with $V_{1}, V_{2}>0$ and $\tan \beta_{V}=V_{1} / V_{2}$.

\subsubsection{Superpotential (a)}

We will begin our discussion with superpotential (a) which turns out to be the most complicated case since it has the most parameters. As it contains two mass parameters, we introduce a second angle $\beta_{M}$ and mean mass $M>0$ such that $M_{24}=M \mathrm{e}^{\mathrm{i} \alpha_{1}} \sin \beta_{M}$ and $M_{24}^{\prime}=M \mathrm{e}^{\mathrm{i} \alpha_{2}} \cos \beta_{M}$. The vacuum expectation values are given by

$$
V_{1} \mathrm{e}^{\mathrm{i} \phi_{1}}=\frac{4 M_{24}^{\prime}}{\kappa^{\prime}} \text { and } V_{2}^{2} \mathrm{e}^{2 \mathrm{i} \phi_{2}}=4 M_{24}^{\prime} \frac{2 M_{24} \kappa^{\prime}-3 M_{24}^{\prime} \lambda}{\kappa^{\prime 3}},
$$

which can as well be expressed in terms of a ratio of the coupling constants involved, $3 \lambda / \kappa^{\prime}=2 \mathrm{e}^{\mathrm{i}\left(\alpha_{1}-\alpha_{2}\right)} \tan \beta_{M}-\mathrm{e}^{-2 \mathrm{i}\left(\phi_{1}-\phi_{2}\right)} \cot ^{2} \beta_{V}$. For the geometric means of the masses of the additional fields compared to the MSSM, we find

$$
\begin{aligned}
& M_{T^{(24)}}^{2}=5 M^{2} \cos \beta_{M} \sqrt{\left(2 \cos \beta_{M}-3 \sin \beta_{M} \tan ^{2} \beta_{V}\right)^{2}+\Delta}, \\
& M_{O^{(24)}}^{2}=5 M^{2} \cos \beta_{M} \sqrt{\left(3 \cos \beta_{M}-2 \sin \beta_{M} \tan ^{2} \beta_{V}\right)^{2}+\Delta}, \\
& M_{L^{(24)}}^{2}=\frac{1}{4} M^{2} \frac{\cos ^{2} \beta_{M}}{\sin ^{4} \beta_{V}},
\end{aligned}
$$

with $\Delta=12 \sin \left(2 \beta_{M}\right) \cot ^{2}\left(\beta_{V}\right) \sin ^{2} \bar{\phi}$ and $\bar{\phi}=\left(\alpha_{1}-\alpha_{2}\right) / 2+\phi_{1}-\phi_{2}$. Note that not only the geometric mean masses, but also the mass eigenvalues themselves only depend on this phase combination $\bar{\phi}$ and are invariant under $\bar{\phi} \rightarrow \bar{\phi}+\pi$.

The effective triplet mass as of eq. (3.8) is heaviest if the phase $\bar{\phi}$ is $0, \pi$ or $2 \pi$, since then the ratio $M_{T^{(24)}} / M_{O^{(24)}}$ is not bounded from above (or below), which allows for the maximal range for $M_{T}^{\operatorname{dim}=5}$. Thus, in the following, we choose $\bar{\phi}$ and thus $\Delta$ to vanish.

The resulting plots for $M_{T}^{\mathrm{dim}=5}$ and for $M_{\mathrm{GUT}}$ are shown in figure 4 for $M_{\mathrm{SUSY}}=1 \mathrm{TeV}$, $M=10^{15} \mathrm{GeV}, M_{D^{(5)}}=1000 \mathrm{TeV}$ and $\bar{\phi}=0$, including a comparison between one- and two-loop results. Note however, that gauge coupling unification depends only weakly on $M_{D^{(5)}} . M_{T}^{\mathrm{dim}=6}$ and $M_{\bar{T}}^{\mathrm{dim}=6}$ are again approximately given by $\sqrt{M_{T}^{\mathrm{dim}=5} M_{D^{(5)}}}$.

\subsubsection{Superpotential (b) and (c)}

These two superpotentials have only one massive parameter $\tilde{M}_{24}=M \mathrm{e}^{\mathrm{i} \alpha}$ and hence the analytic results become much less cumbersome. The vacuum solutions are given by

$$
V_{1} \mathrm{e}^{\mathrm{i} \phi_{1}}=\frac{2}{3} \frac{M \mathrm{e}^{\mathrm{i} \alpha}}{\sqrt[3]{\lambda^{2} \lambda^{\prime}}} \quad \text { and } \quad V_{2} \mathrm{e}^{\mathrm{i} \phi_{2}}=\frac{2}{3} \frac{M \mathrm{e}^{\mathrm{i} \alpha}}{\sqrt[3]{\lambda \lambda^{\prime 2}}}
$$



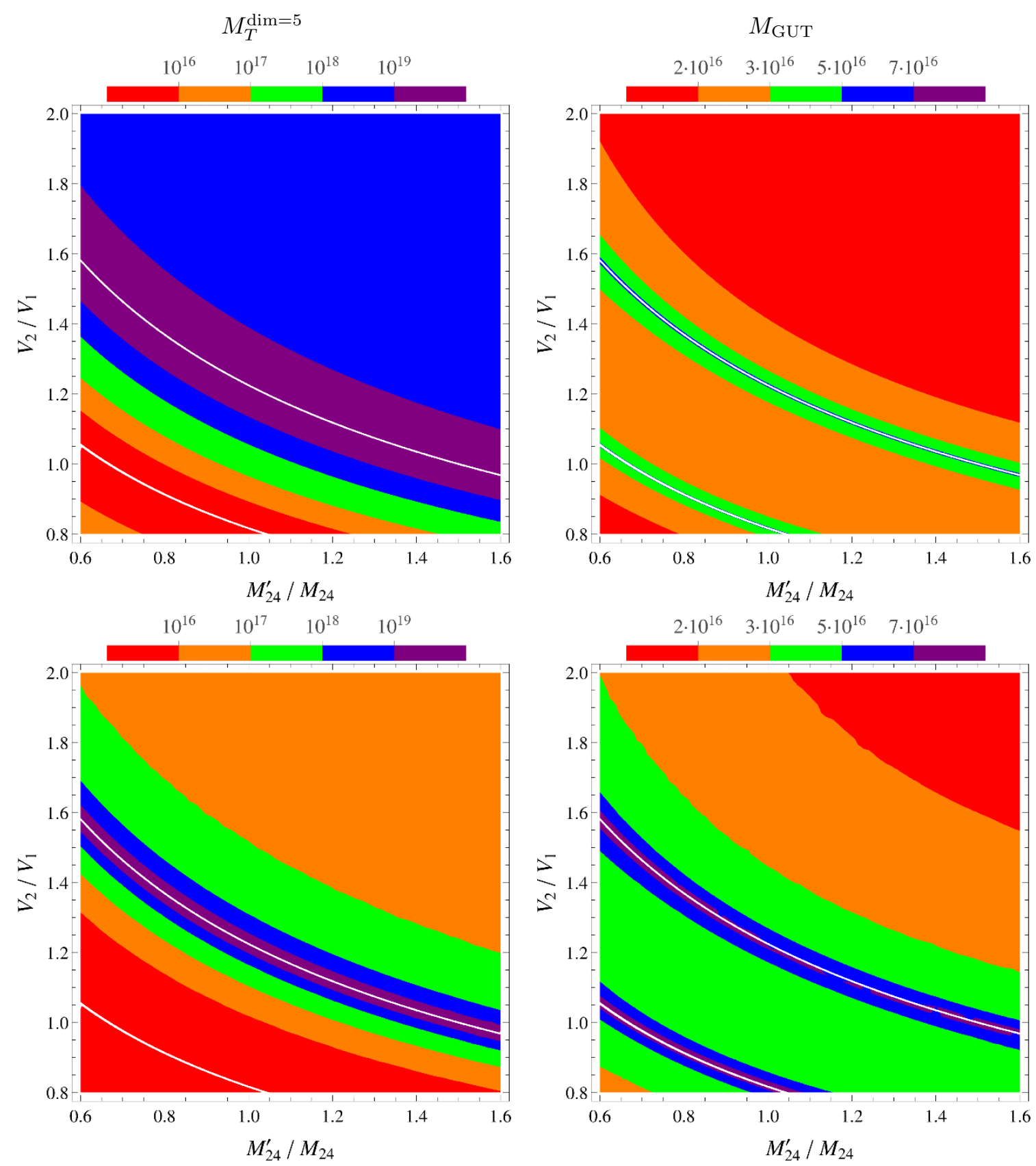

Figure 4. The effective colour triplet mass $M_{T}^{\text {dim }=5}$ (left) and GUT scale $M_{\mathrm{GUT}}$ (right) in GeV at one-loop (upper) and two-loop (lower) order as resulting from superpotential (a) for $M_{\mathrm{SUSY}}=1 \mathrm{TeV}$, $M=10^{15} \mathrm{GeV}, M_{D^{(5)}}=1000 \mathrm{TeV}$ and $\bar{\phi}=0$. Note the different colour coding between left and right. For illustration, the white strips denote areas with light $M_{T^{(24)}}$ or $M_{O^{(24)}}\left(<10^{13} \mathrm{GeV}\right)$. Such relatively low values for these components can arise either from cancellation between terms, or from a generic suppression due to small parameters, cf. eqs. (3.14).

up to a $\mathbb{Z}_{3}$ symmetry transformation for superpotential (b) and

$$
V_{1} \mathrm{e}^{\mathrm{i} \phi_{1}}=\frac{1}{\sqrt{3}} \frac{M \mathrm{e}^{\mathrm{i} \alpha}}{\sqrt{\lambda \kappa^{\prime}}} \quad \text { and } \quad V_{2} \mathrm{e}^{\mathrm{i} \phi_{2}}=\frac{M \mathrm{e}^{\mathrm{i} \alpha}}{\kappa^{\prime}}
$$


up to a minus sign in $V_{1}$ for superpotential (c). In other words, the couplings fulfill the relation $\lambda^{\prime} / \lambda=\mathrm{e}^{3 \mathrm{i}\left(\phi_{2}-\phi_{1}\right)} \tan ^{3} \beta_{V}$ for superpotential (b) and $\kappa^{\prime} / \lambda=3 \mathrm{e}^{2 \mathrm{i}\left(\phi_{1}-\phi_{2}\right)} \tan ^{2} \beta_{V}$ for superpotential (c).

For the geometric means of the masses of the colour triplets, octets and the mass of the left-over leptoquark superfield in $H_{24}$ and $H_{24}^{\prime}$, we find

$$
M_{T^{(24)}}^{2}=\frac{35}{4} M^{2}, \quad M_{O^{(24)}}^{2}=\frac{15}{4} M^{2} \quad \text { and } \quad M_{L^{(24)}}^{2}=\frac{1}{\sin ^{2}\left(2 \beta_{V}\right)} M^{2},
$$

for superpotential (b) and

$$
M_{T^{(24)}}^{2}=\frac{5}{4} M^{2}, \quad M_{O^{(24)}}^{2}=\frac{5}{4} M^{2} \quad \text { and } \quad M_{L^{(24)}}^{2}=\frac{1}{4 \sin ^{2}\left(2 \beta_{V}\right)} M^{2},
$$

for superpotential (c). Note that in both cases also all mass eigenvalues turn out to be phase independent. Therefore, applying eq. (3.8) unification of the gauge couplings on one-loop implies

$$
M_{T}^{\text {dim }=5,1 \text {-loop }}=2.5 \cdot 10^{17} \mathrm{GeV}\left(\frac{M_{\mathrm{SUSY}}}{1 \mathrm{TeV}}\right)^{\frac{5}{6}} \times \begin{cases}\left(\frac{7}{3}\right)^{\frac{5}{2}} \approx 8.3 & \text { (b) } \\ 1 & \text { (c) },\end{cases}
$$

and

$$
M_{\mathrm{GUT}}^{1 \text {-loop }}=\frac{1.37 \cdot 10^{16} \mathrm{GeV}}{\sqrt{\left|\sin 2 \beta_{V}\right|}}\left(\frac{M_{\mathrm{SUSY}}}{1 \mathrm{TeV}}\right)^{-\frac{1}{3}}\left(\frac{M}{10^{15} \mathrm{GeV}}\right)^{-\frac{1}{2}} \times \begin{cases}\sqrt{\frac{8}{\sqrt{21}}} \approx 1.32 & \text { (b) } \\ 2 & \text { (c) } .\end{cases}
$$

Assuming the same parameters, we find an almost ten times heavier effective triplet mass in superpotential (b) than in (c) and hence we focus on this case in our second example model later.

At two-loop, we find the following approximate behaviour for the masses

$$
M_{T}^{\text {dim=5, 2-loop }}=\left(\frac{M_{\text {SUSY }}}{1 \mathrm{TeV}}\right)^{0.74} \cdot \begin{cases}5.2 \cdot 10^{16} \mathrm{GeV}\left(\frac{M}{10^{15} \mathrm{GeV}}\right)^{-0.15} & \text { (b) } \\ 6.8 \cdot 10^{15} \mathrm{GeV}\left(\frac{M}{10^{15} \mathrm{GeV}}\right)^{-0.18} & \text { (c) },\end{cases}
$$

and

$$
M_{\mathrm{GUT}}^{2 \text {-loop }}=\left|\sin 2 \beta_{V}\right|^{-0.48}\left(\frac{M_{\mathrm{SUSY}}}{1 \mathrm{TeV}}\right)^{-0.4} \cdot \begin{cases}2.89 \cdot 10^{16} \mathrm{GeV}\left(\frac{M}{10^{15} \mathrm{GeV}}\right)^{-0.61} & \text { (b) } \\ 4.78 \cdot 10^{16} \mathrm{GeV}\left(\frac{M}{10^{15} \mathrm{GeV}}\right)^{-0.63} & \text { (c) } .\end{cases}
$$

The dependence on other parameters is very small. As we can see, at two-loop, having $M_{T}^{\text {dim=5 }} \gtrsim 10^{17} \mathrm{GeV}$ requires $M_{\mathrm{SUSY}} \gtrsim 2.3 \mathrm{TeV}$ and $35 \mathrm{TeV}$ for superpotential (b) and (c) respectively. Again, $M_{D^{(5)}}=1000 \mathrm{TeV}$ has been fixed and the values of $M_{T}^{\text {dim=6, 1-loop }}$ and $M_{T}^{\text {dim }=6,2-l o o p}$ can be approximated by the square root of the product of $M_{D^{(5)}}$ and $M_{T}^{\text {dim=5, 1-loop }}$ or $M_{T}^{\text {dim=5, 2-loop }}$ respectively.

There are a few more comments in order. There is a claim [27] that the MSSM with an additional unbroken R-symmetry can not be obtained from the spontaneous breaking of a 
four-dimensional (SUSY) GUT. Note that this is not in conflict with our superpotentials, because the R-symmetry is either absent ( $\mathrm{a}, \mathrm{b}, \mathrm{d})$ or spontaneously broken at the GUT scale (c). Superpotential (c) is particularly interesting for model building purposes because R-symmetries are very popular in flavour models with non-Abelian family symmetries (and spontaneous CP violation). We will discuss this in more detail in appendix D.

\section{Flavour models with DMPM}

In this section we combine the DMPM (featuring two adjoints of SU(5)) with a predictive GUT flavour model for the quark-lepton Yukawa ratios at the GUT scale. In particular, we implement CG factors as given in [11]. Two examples with different Yukawa matrix structures are presented: in the first model we construct diagonal down-type quark and charged lepton Yukawa matrices $Y_{d}$ and $Y_{e}$, with all mixing originating from the up-type quark Yukawa matrix $Y_{u}$. The second model realizes the attractive feature of the Cabibbo mixing angle $\theta_{C}$ originating from $Y_{d}$. Both models are providing existence proofs that successful DTS and experimentally viable predictions for the GUT scale Yukawa coupling ratios can indeed be realised simultaneously in one model.

Let us be more specific on the predictions made by the two models: due to the CG factors in the down-type quark and charged lepton sector, $\frac{y_{\tau}}{y_{b}}, \frac{y_{\mu}}{y_{s}}$ and $\frac{y_{e}}{y_{d}}$ are predicted at the GUT scale. To confront them with the experimental data, the RG running to low energies has to be performed, including in particular supersymmetric 1-loop threshold corrections [28-31] when the MSSM is matched to the SM. These threshold corrections can have a sizeable impact on the low energy values of the Yukawa couplings (and thus the fermion masses), depending on the sparticle spectrum and $\tan \beta$. So the predictions here are two-fold: firstly, the predictions for the Yukawa ratios at the GUT scale imply constraints on the SUSY breaking parameters, which may be tested at future collider searches if SUSY is found. ${ }^{6}$ Secondly, the ratios $\frac{y_{d}}{y_{s}}$ and $\frac{y_{e}}{y_{\mu}}$ are not affected by RG running and by the SUSY threshold corrections (as long as the first two families of sfermions are almost degenerate in mass as commonly assumed). They can be directly used to constrain GUT models. A particularly useful quantity in this context is indeed the double ratio

$$
\left|\frac{y_{\mu}}{y_{s}} \frac{y_{d}}{y_{e}}\right|=10.7_{-0.8}^{+1.8}
$$

which can be checked directly at the GUT scale [25]. In our models we will use the CG factors $\frac{y_{\tau}}{y_{b}}=-\frac{3}{2}, \frac{y_{\mu}}{y_{s}}=6$ and $\frac{y_{e}}{y_{d}}=-\frac{1}{2}$. This leads to $\left|\frac{y_{\mu}}{y_{s}} \frac{y_{d}}{y_{e}}\right|=12$, which is in good agreement with the phenomenological value. On the other hand, the ubiquitous CG factors $y_{\mu}=-3 y_{s}$ and $y_{e}=\frac{1}{3} y_{d}$, known as Georgi-Jarlskog relations [3], would give $\left|\frac{y_{\mu}}{y_{s}} \frac{y_{d}}{y_{e}}\right|=9$ and deviate from the central value 10.7 by more than two sigma.

In this section we explicitly construct only the Yukawa matrices of the up- and downtype quarks and charged leptons. Adding one of the ubiquitous mechanisms to generate

\footnotetext{
${ }^{6}$ To make explicit statements about the constraints on the SUSY parameters one would have to specify the model of SUSY breaking, which is beyond the scope of this paper. A discussion and an explicit example where such constraints are worked out can be found, e.g. in [32].
} 
neutrino masses and lepton mixing angles would be straightforward. However, we do not consider neutrinos in this paper, since they are not directly relevant for the discussion of proton decay, doublet-triplet splitting and the CG factors between $Y_{d}$ and $Y_{e}$.

\subsection{A model with diagonal $Y_{d}$ and $Y_{e}$ Yukawa matrices}

We now turn to our first model featuring diagonal down-type quark and charged lepton Yukawa matrices $Y_{d}$ and $Y_{e}{ }^{7}$ In this case all the mixing in the quark sector has to come exclusively from the up-type quark Yukawa matrix $Y_{u}$. Explicitly, we have the following structure for the Yukawa matrices

$$
Y_{d}=\left(\begin{array}{ccc}
y_{d} & 0 & 0 \\
0 & y_{s} & 0 \\
0 & 0 & y_{b}
\end{array}\right), \quad Y_{e}=\left(\begin{array}{ccc}
-\frac{1}{2} y_{d} & 0 & 0 \\
0 & 6 y_{s} & 0 \\
0 & 0 & -\frac{3}{2} y_{b}
\end{array}\right), \quad Y_{u}=\left(\begin{array}{lll}
y_{11} & y_{12} & y_{13} \\
y_{12} & y_{22} & y_{23} \\
y_{13} & y_{23} & y_{33}
\end{array}\right) .
$$

An approach to flavour (GUT) model building with diagonal $Y_{e}$ (and $Y_{d}$ ) has been discussed recently in [33, 34].

We introduce flavon fields $\theta_{1}, \theta_{2}, \theta_{3}$ and $\theta_{4}$ that obtain a VEV and generate the hierarchical structure of the Yukawa matrices. After the flavon fields, $H_{24}$ and $H_{24}^{\prime}$ obtain their VEVs, the Yukawa matrices of eq. (4.2) originate from the following effective superpotentials

$$
\begin{aligned}
W_{u}= & \frac{1}{\Lambda^{4}} H_{5} \mathcal{T}_{1} \mathcal{T}_{1} \theta_{1}^{2} \theta_{2}^{2}+\frac{1}{\Lambda^{3}} H_{5} \mathcal{T}_{1} \mathcal{T}_{2} \theta_{1}^{2} \theta_{2}+\frac{1}{\Lambda^{2}} H_{5} \mathcal{T}_{1} \mathcal{T}_{3} \theta_{1} \theta_{2}+\frac{1}{\Lambda^{2}} H_{5} \mathcal{T}_{2} \mathcal{T}_{2} \theta_{1}^{2} \\
& +\frac{1}{\Lambda} H_{5} \mathcal{T}_{2} \mathcal{T}_{3} \theta_{1}+H_{5} \mathcal{T}_{3} \mathcal{T}_{3}, \\
W_{d}= & \frac{1}{\left\langle S^{\prime}\right\rangle}\left(H_{24}^{\prime} \mathcal{F}_{3}\right)_{5}\left(\bar{H}_{5} \mathcal{T}_{3}\right)_{5}+\frac{\theta_{3}}{\left\langle S^{\prime}\right\rangle^{2}}\left(H_{24}^{\prime} \mathcal{T}_{2}\right)_{10}\left(\bar{H}_{5} \mathcal{F}_{2}\right)_{\overline{10}} \\
& +\frac{\theta_{4}}{\left\langle S^{\prime}\right\rangle^{2}\langle S\rangle}\left(H_{24}^{\prime} \mathcal{F}_{1}\right)_{\overline{45}}\left(\mathcal{T}_{1} H_{24} \bar{H}_{5}\right)_{45},
\end{aligned}
$$

where we do not show order one coefficients, and denote the different messenger masses generating $W_{u}$ by a generic $\Lambda$. However, keep in mind that this is just for the sake of simplicity and different entries in the Yukawa matrix should be understood as independent parameters. The ratios of flavon VEVs and messenger masses is small of about 0.010.1. For a list of all fields including their charges under the additional discrete shaping symmetries, see tables $2,3,4$, and 5 .

The adjoint $H_{24}^{\prime}$ required to construct the desired CG factors must be charged under shaping symmetries. We will therefore implement superpotential (a). Note that it leaves the second adjoint $H_{24}$ uncharged, which could in principle lead to a problem. A direct mass term of messenger fields $M_{i} Z_{i} \bar{Z}_{i}$ would in this case always show a up with a term of the form $H_{24} Z_{i} \bar{Z}_{i}$. Such a contribution would inevitably spoil the desired CG factors [22] between $Y_{d}$ and $Y_{e}^{T}$ as long as the mass and the adjoint VEV are not very hierarchical. To avoid this and still generate the desired operators the masses of the messenger fields that

\footnotetext{
${ }^{7}$ The matrices are diagonal in the preferred basis where the different fermion generations have well defined symmetry assignments, cf. table 2 .
} 


\begin{tabular}{|cccccccccc|}
\hline & $\mathrm{SU}(5)$ & $\mathbb{Z}_{2}$ & $\mathbb{Z}_{4}$ & $\mathbb{Z}_{4}$ & $\mathbb{Z}_{4}$ & $\mathbb{Z}_{7}$ & $\mathbb{Z}_{7}$ & $\mathbb{Z}_{9}$ & $\mathbb{Z}_{2}$ \\
\hline$H_{5}$ & $\mathbf{5}$ &. &. &. &. &. &. &. &. \\
$\bar{H}_{5}$ & $\overline{\mathbf{5}}$ &. & 2 &. &. &. & 1 & 2 &. \\
$\mathcal{T}_{1}$ & $\mathbf{1 0}$ &. &. & 3 &. & 6 &. &. & 1 \\
$\mathcal{T}_{2}$ & $\mathbf{1 0}$ &. &. &. &. & 6 &. &. & 1 \\
$\mathcal{T}_{3}$ & $\mathbf{1 0}$ &. &. &. &. &. &. &. & 1 \\
$\mathcal{F}_{1}$ & $\overline{\mathbf{5}}$ & 1 & 1 & 1 & 2 & 1 & 1 & 2 & 1 \\
$\mathcal{F}_{2}$ & $\overline{\mathbf{5}}$ & 1 &. &. & 2 & 1 &. & 2 & 1 \\
$\mathcal{F}_{3}$ & $\overline{\mathbf{5}}$ & 1 & 2 &. & 1 &. & 6 & 7 & 1 \\
\hline
\end{tabular}

Table 2. SU(5) representations and charges under discrete shaping symmetries of the MSSM fields and colour triplets of the model presented in subsection 4.1. A dot denotes charge zero.

\begin{tabular}{|cccccccccc|}
\hline & $\mathrm{SU}(5)$ & $\mathbb{Z}_{2}$ & $\mathbb{Z}_{4}$ & $\mathbb{Z}_{4}$ & $\mathbb{Z}_{4}$ & $\mathbb{Z}_{7}$ & $\mathbb{Z}_{7}$ & $\mathbb{Z}_{9}$ & $\mathbb{Z}_{2}$ \\
\hline$H_{24}$ & $\mathbf{2 4}$ &. &. &. &. &. &. &. &. \\
$H_{24}^{\prime}$ & $\mathbf{2 4}$ & 1 &. &. &. &. &. &. &. \\
$S$ & $\mathbf{1}$ &. & 3 &. &. &. & 2 &. &. \\
$S^{\prime}$ & $\mathbf{1}$ &. &. &. & 1 &. &. &. &. \\
$\theta_{1}$ & $\mathbf{1}$ &. &. &. &. & 1 &. &. &. \\
$\theta_{2}$ & $\mathbf{1}$ &. &. & 1 &. &. &. &. &. \\
$\theta_{3}$ & $\mathbf{1}$ &. & 2 &. &. &. & 6 & 5 &. \\
$\theta_{4}$ & $\mathbf{1}$ &. &. &. &. &. &. & 5 &. \\
\hline
\end{tabular}

Table 3. SU(5) representations and charges under discrete shaping symmetries of the superfields obtaining VEVs at around the GUT scale of the model presented in subsection 4.1. A dot denotes charge zero.

give rise to $W_{d}$ in eq. (4.4) originate from the VEVs of the fields $S$ and $S^{\prime}$ charged under the shaping symmetry (but with different charges than $H_{24}^{\prime}$ ) ${ }^{8}$

The full messenger sector can be read off from the supergraphs presented in figures 5 and 6 , see also table 5. After the heavy messenger fields get integrated out, the effective superpotentials $W_{d}$ and $W_{u}$ are obtained.

The predictivity of the down-type quarks and charged lepton Yukawa couplings has already been discussed above. Note that the hierarchical structure is enforced due to the use of higher order effective operators in $W_{d}$. In a small angle approximation the leading order estimates for the eigenvalues of $Y_{u}$ and the mixing angles are given as

$$
y_{u} \approx y_{11}-\frac{y_{12}^{2}}{y_{22}}, \quad y_{c} \approx y_{22}, \quad y_{t} \approx y_{33}, \quad \theta_{C} \approx \frac{y_{12}}{y_{22}}, \quad \theta_{23} \approx \frac{y_{23}}{y_{33}}, \quad \theta_{13} \approx \frac{y_{13}}{y_{33}} .
$$

Phenomenology requires that all parameters $y_{i j}$ of $Y_{u}$ are independent, which needs to be carefully considered in the construction of the messenger sector, as discussed in appendix $\mathrm{C}$.

\footnotetext{
${ }^{8}$ The VEV of the charged singlet $S$ gives masses to the heavy messengers of the DMPM (see section 2.5).
} 


\begin{tabular}{|cccccccccc|}
\hline & $\mathrm{SU}(5)$ & $\mathbb{Z}_{2}$ & $\mathbb{Z}_{4}$ & $\mathbb{Z}_{4}$ & $\mathbb{Z}_{4}$ & $\mathbb{Z}_{7}$ & $\mathbb{Z}_{7}$ & $\mathbb{Z}_{9}$ & $\mathbb{Z}_{2}$ \\
\hline$H_{5}^{\prime}$ & $\mathbf{5}$ &. & 3 &. &. &. & 5 & 7 &. \\
$\bar{H}_{5}^{\prime}$ & $\overline{\mathbf{5}}$ &. & 1 &. &. &. & 6 &. &. \\
$X_{45}$ & $\mathbf{4 5}$ &. & 2 &. &. &. & 6 & 7 &. \\
$\bar{X}_{45}$ & $\overline{\mathbf{4 5}}$ &. & 3 &. &. &. & 6 & 2 &. \\
$Y_{45}$ & $\mathbf{4 5}$ &. &. &. &. &. & 3 & 7 &. \\
$\bar{Y}_{45}$ & $\overline{\mathbf{4 5}}$ &. & 1 &. &. &. & 2 & 2 &. \\
$Z_{50}$ & $\mathbf{5 0}$ &. & 1 &. &. &. & 1 & 7 &. \\
$\bar{Z}_{50}$ & $\overline{\mathbf{5 0}}$ &. &. &. &. &. & 4 & 2 &. \\
$X_{45}^{\prime}$ & $\mathbf{4 5}$ &. & 3 &. &. &. & 1 &. &. \\
$\bar{X}_{45}^{\prime}$ & $\overline{\mathbf{4 5}}$ &. & 2 &. &. &. & 4 &. &. \\
$Y_{45}^{\prime}$ & $\mathbf{4 5}$ &. & 1 &. &. &. & 5 &. &. \\
$\bar{Y}_{45}^{\prime}$ & $\overline{\mathbf{4 5}}$ &. &. &. &. &. &. &. &. \\
$Z_{50}^{\prime}$ & $\mathbf{5 0}$ &. & 2 &. &. &. & 3 &. &. \\
$\bar{Z}_{50}^{\prime}$ & $\overline{\mathbf{5 0}}$ &. & 3 &. &. &. & 2 &. &. \\
\hline
\end{tabular}

Table 4. SU(5) representations and charges under discrete shaping symmetries of the fields in the DMPM sector of the model presented in subsection 4.1. A dot denotes charge zero.

\begin{tabular}{|cccccccccc|}
\hline & $\mathrm{SU}(5)$ & $\mathbb{Z}_{2}$ & $\mathbb{Z}_{4}$ & $\mathbb{Z}_{4}$ & $\mathbb{Z}_{4}$ & $\mathbb{Z}_{7}$ & $\mathbb{Z}_{7}$ & $\mathbb{Z}_{9}$ & $\mathbb{Z}_{2}$ \\
\hline$Z_{5,1}$ & $\mathbf{5}$ &. & 2 &. & 3 &. & 1 & 2 & 1 \\
$\bar{Z}_{5,1}$ & $\overline{\mathbf{5}}$ &. & 2 &. &. &. & 6 & 7 & 1 \\
$Z_{10,1}$ & $\mathbf{1 0}$ & 1 &. &. & 3 & 6 &. &. & 1 \\
$\bar{Z}_{10,1}$ & $\overline{\mathbf{1 0}}$ & 1 &. &. &. & 1 &. &. & 1 \\
$Z_{10,2}$ & $\mathbf{1 0}$ & 1 & 2 &. & 2 & 6 & 6 & 5 & 1 \\
$\bar{Z}_{10,2}$ & $\overline{\mathbf{1 0}}$ & 1 & 2 &. & 1 & 1 & 1 & 4 & 1 \\
$Z_{45,1}$ & $\mathbf{4 5}$ &. & 3 & 3 & 2 & 6 & 6 & 7 & 1 \\
$\bar{Z}_{45,1}$ & $\overline{\mathbf{4 5}}$ &. & 1 & 1 & 1 & 1 & 1 & 2 & 1 \\
$Z_{45,2}$ & $\mathbf{4 5}$ &. & 3 & 3 & 3 & 6 & 6 & 2 & 1 \\
$\bar{Z}_{45,2}$ & $\overline{\mathbf{4 5}}$ &. & 1 & 1 &. & 1 & 1 & 7 & 1 \\
\hline$Z_{10,3}$ & $\mathbf{1 0}$ &. &. &. &. & 1 &. &. & 1 \\
$Z_{10,4}$ & $\mathbf{1 0}$ &. &. & 1 &. & 1 &. &. & 1 \\
$Z_{1}$ & $\mathbf{1}$ &. &. &. &. & 5 &. &. &. \\
$Z_{2}$ & $\mathbf{1}$ &. &. & 3 &. & 5 &. &. &. \\
\hline
\end{tabular}

Table 5. SU(5) representations and charges under discrete shaping symmetries of the flavon and flavour messenger fields of the model presented in subsection 4.1. Note that the messengers $Z_{5,1} \bar{Z}_{5,1}, Z_{10,1} \bar{Z}_{10,1}, Z_{10,2} \bar{Z}_{10,2}, Z_{45,1} \bar{Z}_{45,1}$ and $Z_{45,2} \bar{Z}_{45,2}$ have no direct mass term, but get their masses through the VEVs of $S$ and $S^{\prime}$. The other messenger fields have direct mass terms, so their corresponding barred field is omitted in this table. A dot denotes charge zero. 


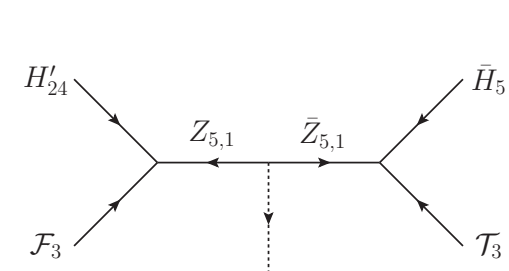

$\left\langle S^{\prime}\right\rangle$

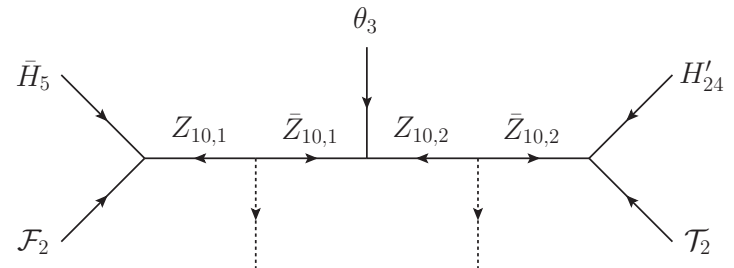

$\left\langle S^{\prime}\right\rangle$

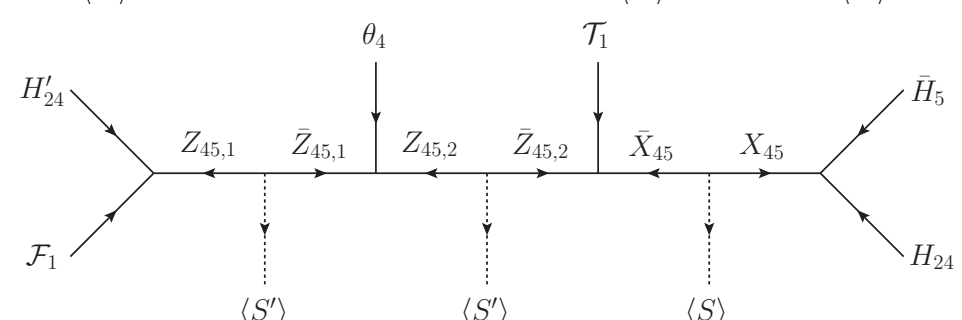

$\left\langle S^{\prime}\right\rangle$

$\left\langle S^{\prime}\right\rangle$

$\langle S\rangle$

Figure 5. Supergraphs leading to the effective superpotential $W_{d}$ of eq. (4.4) when the heavy messenger fields get integrated out in the model presented in subsection 4.1.

In section 2.5 we argued that for the case of an uncharged $H_{24}$, as it appears in the selected superpotential (a), the mass term for the additional, five-dimensional Higgs fields must come from the VEV of some singlet field. In our model an effective $\mu^{\prime}$ term is generated from a higher-dimensional operator and with an even higher suppression, there is also a $\mu$-term for the Higgs fields coupling to matter,

$$
W_{5}^{\mathrm{eff}}=\mu H_{5} \bar{H}_{5}+\mu^{\prime} H_{5}^{\prime} \bar{H}_{5}^{\prime}
$$

where $\mu^{\prime} \equiv\left\langle\theta_{3}\right\rangle^{4} / M_{\mathrm{Pl}}^{3}$ and $\mu \equiv\left\langle\theta_{3}\right\rangle\left\langle\theta_{4}\right\rangle^{4} / M_{\mathrm{Pl}}^{4}{ }^{9}$. The mass matrices for the doublet and triplet components are then given by

$$
m_{D}=\left(\begin{array}{cc}
\mu & 0 \\
0 & \mu^{\prime}
\end{array}\right), \quad m_{T}=\left(\begin{array}{cccc}
\mu & 0 & 0 & -\frac{V_{1}^{2}}{\langle S\rangle} \\
0 & \mu^{\prime} & -\frac{V_{1}^{2}}{\langle S\rangle} & 0 \\
-\frac{V_{1}^{2}}{\langle S\rangle} & 0 & \langle S\rangle & 0 \\
0 & -\frac{V_{1}^{2}}{\langle S\rangle} & 0 & \langle S\rangle
\end{array}\right),
$$

where $V_{1}$ is defined in eq. (3.11). After the heavy 50-dimensional fields are integrated out and $\mathrm{SU}(5)$ gets spontaneously broken, the mass matrices for the doublet and triplet components of the Higgs fields $H_{5}, H_{5}^{\prime}$ and their corresponding barred fields are given by

$$
m_{D}=\left(\begin{array}{cc}
\mu & 0 \\
0 & \mu^{\prime}
\end{array}\right), \quad m_{T}=\left(\begin{array}{cc}
\mu & -\frac{V_{1}^{4}}{\langle S\rangle^{3}} \\
-\frac{V_{1}^{4}}{\langle S\rangle^{3}} & \mu^{\prime}
\end{array}\right) .
$$

The effective triplet mass for dimension five proton decay is then given by

$$
M_{T}^{\operatorname{dim}=5}=\left(m_{T}^{-1}\right)_{11}^{-1}=\mu-\frac{V_{1}^{8}}{\langle S\rangle^{6} \mu^{\prime}} \approx-\frac{V_{1}^{8} M_{\mathrm{Pl}}^{3}}{\langle S\rangle^{6}\left\langle\theta_{3}\right\rangle^{4}} .
$$

\footnotetext{
${ }^{9}$ Alternatively, we checked that a UV-complete generation of these $\mu$ and $\mu^{\prime}$ operators via messenger fields would be possible. However, the necessary messenger fields are not included in the model and it turns out that the masses are already generated by Planck-scale suppressed effective operators.
} 


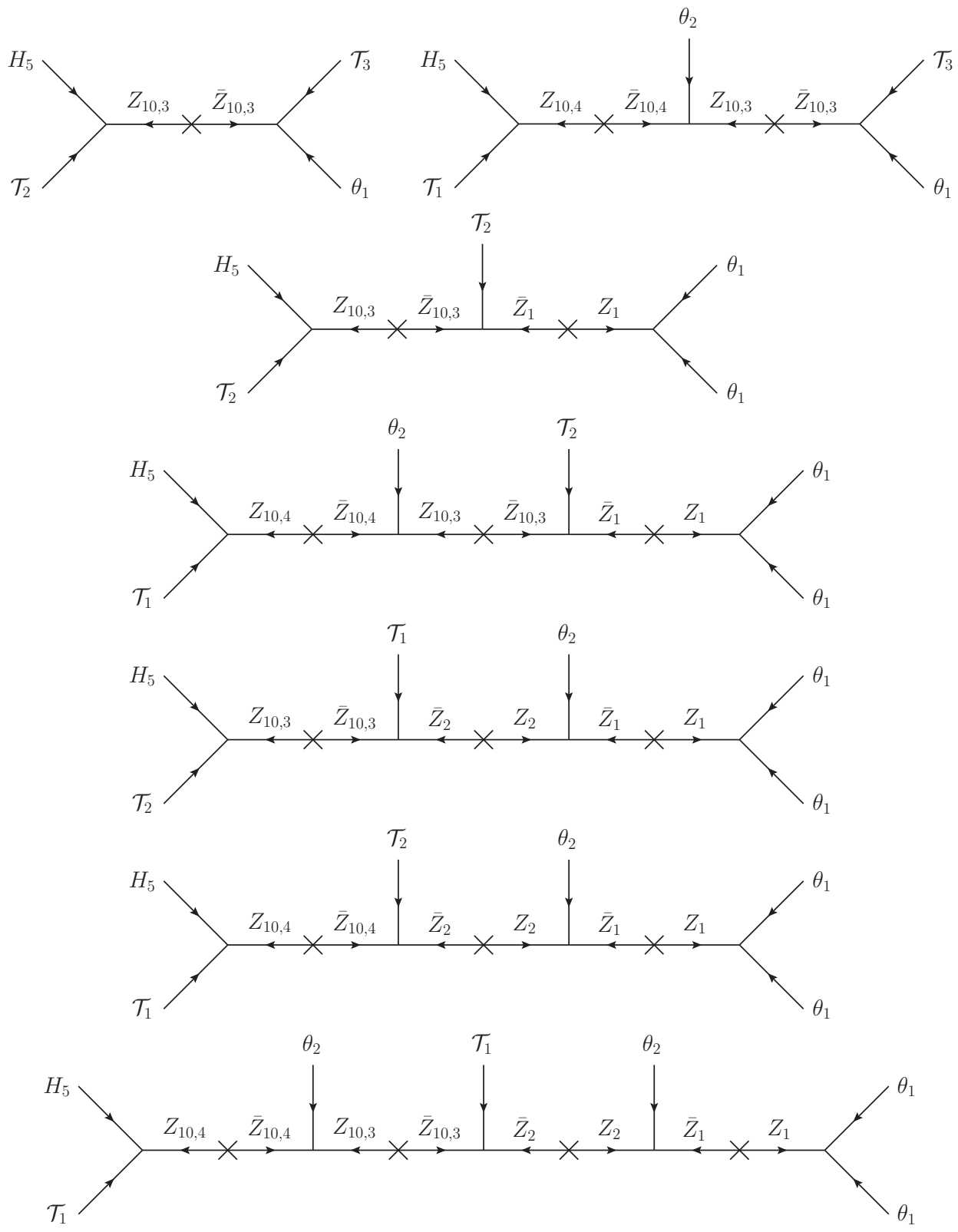

Figure 6. Supergraphs leading to the effective superpotential $W_{u}$ of eq. (4.3) when the heavy messenger fields are integrated out in the model presented in subsection 4.1. Note that there are three supergraphs contributing to the superpotential term generating $y_{12}$. A detailed discussion of the messenger sector is presented in the appendix $\mathrm{C}$.

and the effective triplet masses suppressing dimension six proton decay are given by

$$
\left(M_{T}^{\operatorname{dim}=6}\right)^{2}=\left(M_{\bar{T}}^{\operatorname{dim}=6}\right)^{2} \approx \frac{\left|V_{1}\right|^{8}}{|\langle S\rangle|^{6}} .
$$

Let us give an explicit example for the scales involved in the model: because of perturbativity the mass of the 50-dimensional Higgs fields has to be almost at the Planck scale. 
We therefore assume $\langle S\rangle \sim 10^{-1} M_{\mathrm{Pl}}$. Using the known values of the Yukawa couplings, we can estimate the values of the relevant masses of our model. At the GUT scale with $\tan \beta=30$ the Yukawa couplings are approximately given by [25]

$$
y_{d} \approx 1.6 \cdot 10^{-4}, \quad y_{s} \approx 3 \cdot 10^{-3}, \quad \text { and } \quad y_{b} \approx 0.18 \text {. }
$$

In our example model these Yukawa couplings are (up to order one couplings) given by the operators

$$
y_{d} \sim \frac{\left\langle H_{24}\right\rangle\left\langle H_{24}^{\prime}\right\rangle\left\langle\theta_{4}\right\rangle}{\left\langle S^{\prime}\right\rangle^{2}\langle S\rangle}, \quad y_{s} \sim \frac{\left\langle H_{24}^{\prime}\right\rangle\left\langle\theta_{3}\right\rangle}{\left\langle S^{\prime}\right\rangle^{2}} \quad \text { and } \quad y_{b} \sim \frac{\left\langle H_{24}^{\prime}\right\rangle}{\left\langle S^{\prime}\right\rangle} .
$$

Then we find from eqs. (4.9)-(4.12) for the effective triplet masses, the $\mu$-term and the mass of the additional heavy doublet the following values

$$
M_{T}^{\operatorname{dim}=5} \approx 1.4 \cdot 10^{19} \mathrm{GeV}, \quad M_{T}^{\operatorname{dim}=6} \approx 1.4 \cdot 10^{12} \mathrm{GeV}, \quad \mu \approx 225 \mathrm{GeV}, \quad \mu^{\prime} \approx 130 \mathrm{TeV}
$$

Recalling from section 3.1.1 the parameters governing superpotential (a) and using here SUSY scale of $M_{\mathrm{SUSY}}=1 \mathrm{TeV}, \lambda \sim 0.19, \kappa^{\prime} \sim 0.08, M_{24}^{\prime}=M_{24}=10^{15} \mathrm{GeV}$ and $V_{2} / V_{1}=$ 1.2 the GUT scale is given by

$$
M_{\mathrm{GUT}} \approx 6.4 \cdot 10^{16} \mathrm{GeV} \text {. }
$$

Although these numbers are only estimates, which neglect order one couplings, they illustrate the model's features: the DTS problem is solved with large effective triplet masses, therefore the proton decay rate is suppressed and the fermion mass ratios are realistic. The $\mu$-term emerges from a Planck-scale suppressed operator. We remark that the DMPM does not suffer from any dangerous Planck-scale suppressed operators, due to the charge assignment of the singlet field $S$.

\subsection{A model with $\theta_{C}$ from $Y_{d}$}

We now turn to our second example model, which realises the attractive feature of $\theta_{C}$ emerging dominantly from the down-type quark mixing $\theta_{C} \approx \theta_{12}^{d}$. The Yukawa matrices are given by the following structure

$$
Y_{d}=\left(\begin{array}{ccc}
0 & y_{d, 12} & 0 \\
y_{d, 21} & y_{s} & 0 \\
0 & 0 & y_{b}
\end{array}\right), \quad Y_{e}=\left(\begin{array}{ccc}
0 & 6 y_{d, 21} & 0 \\
-\frac{1}{2} y_{d, 12} & 6 y_{s} & 0 \\
0 & 0 & -\frac{3}{2} y_{b}
\end{array}\right), \quad Y_{u}=\left(\begin{array}{ccc}
y_{11} & y_{12} & 0 \\
y_{12} & y_{22} & y_{23} \\
0 & y_{23} & y_{33}
\end{array}\right)
$$

A similar structure $[16,17]$ has been used to explain the relation $\theta_{13}^{\text {PMNS }}=\theta_{C} / \sqrt{2}$ via charged lepton corrections, and a right-handed quark unitarity triangle [35]. From the matrices we can see that we want to couple both $\mathcal{F}_{1}$ and $\mathcal{F}_{2}$ to $\mathcal{T}_{2}$ but still distinguish both fields from each other to forbid $\left(Y_{d}\right)_{11}$. This suggests to use at least a $\mathbb{Z}_{3}$ symmetry and hence we will use superpotential (b) from section 3.1 where both adjoints are charged. The effective superpotentials that lead to the desired Yukawa matrices after integrating 


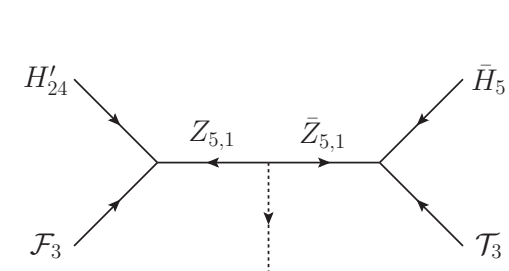

$\left\langle S^{\prime}\right\rangle$

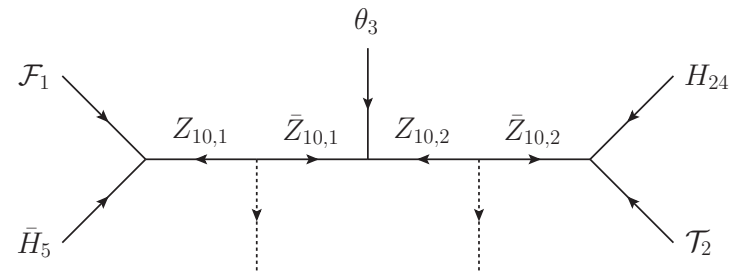

$\left\langle S^{\prime}\right\rangle$

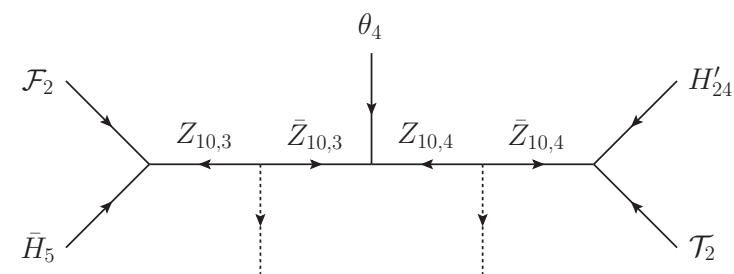

$\left\langle S^{\prime}\right\rangle$

$\left\langle S^{\prime}\right\rangle$

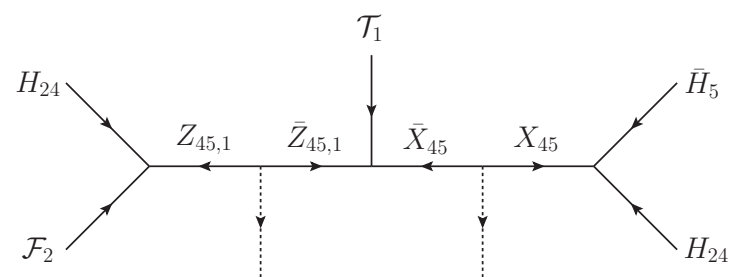

$\langle S\rangle$

$\langle S\rangle$

Figure 7. Supergraphs leading to the effective superpotential $W_{d}$ of eq. (4.17) when the heavy messenger fields get integrated out in the model presented in subsection 4.2.

out heavy messenger fields and breaking of the GUT gauge group are

$$
\begin{aligned}
W_{u}= & \frac{1}{\Lambda^{2}} H_{5} \mathcal{T}_{1} \mathcal{T}_{1} \theta_{5}^{2}+\frac{1}{\Lambda^{2}} H_{5} \mathcal{T}_{1} \mathcal{T}_{2} \theta_{2}^{2}+\frac{1}{\Lambda^{2}} H_{5} \mathcal{T}_{2} \mathcal{T}_{2} \theta_{1}^{2}+\frac{1}{\Lambda} H_{5} \mathcal{T}_{2} \mathcal{T}_{3} \theta_{1}+H_{5} \mathcal{T}_{3} \mathcal{T}_{3} \\
W_{d}= & \frac{1}{\left\langle S^{\prime}\right\rangle}\left(H_{24}^{\prime} \mathcal{F}_{3}\right)_{5}\left(\bar{H}_{5} \mathcal{T}_{3}\right)_{5}+\frac{\theta_{3}}{\left\langle S^{\prime}\right\rangle^{2}}\left(H_{24} \mathcal{T}_{2}\right)_{10}\left(\bar{H}_{5} \mathcal{F}_{1}\right)_{\overline{10}}+\frac{\theta_{4}}{\left\langle S^{\prime}\right\rangle^{2}}\left(H_{24}^{\prime} \mathcal{T}_{2}\right)_{10}\left(\bar{H}_{5} \mathcal{F}_{2}\right)_{\overline{10}} \\
& +\frac{1}{\langle S\rangle^{2}}\left(H_{24} \mathcal{F}_{2}\right)_{\overline{45}}\left(\mathcal{T}_{1} H_{24} \bar{H}_{5}\right)_{45}
\end{aligned}
$$

where again VEVs of singlet fields appear in the denominators by virtue of messenger masses generated by them. Note that in comparison to $Y_{u}$ of the previous example model of eq. (4.2), the vanishing $\left(Y_{u}\right)_{13}$ element requires to introduce an additional flavon field $\theta_{5}$. The supergraphs that generate these effective operators are shown in figure 7 and 8 . A complete list of all fields including their charges and representations is given in tables $6,7,8$ and 9 .

In a small angle approximation the mixing angles and Yukawa couplings are given by

$$
\begin{array}{rlrl}
y_{d} & \approx \frac{y_{d, 12} y_{d, 21}}{y_{s}}, & \theta_{C} & \approx \theta_{12}^{d} \approx \frac{y_{d, 12}}{y_{s}}, \\
y_{u} \approx y_{11}-\frac{y_{12}^{2}}{y_{22}}, & y_{c} \approx y_{22}, \quad y_{t} \approx y_{33}, & \theta_{23} \approx \frac{y_{23}}{y_{33}}, \quad \theta_{13} \approx \frac{y_{12}}{y_{22}} \theta_{23},
\end{array}
$$

with $y_{s}$ and $y_{b}$ given as parameters of $Y_{d}$ in eq. (4.15). Thus the Yukawa matrices can fit the experimental values without tension. 


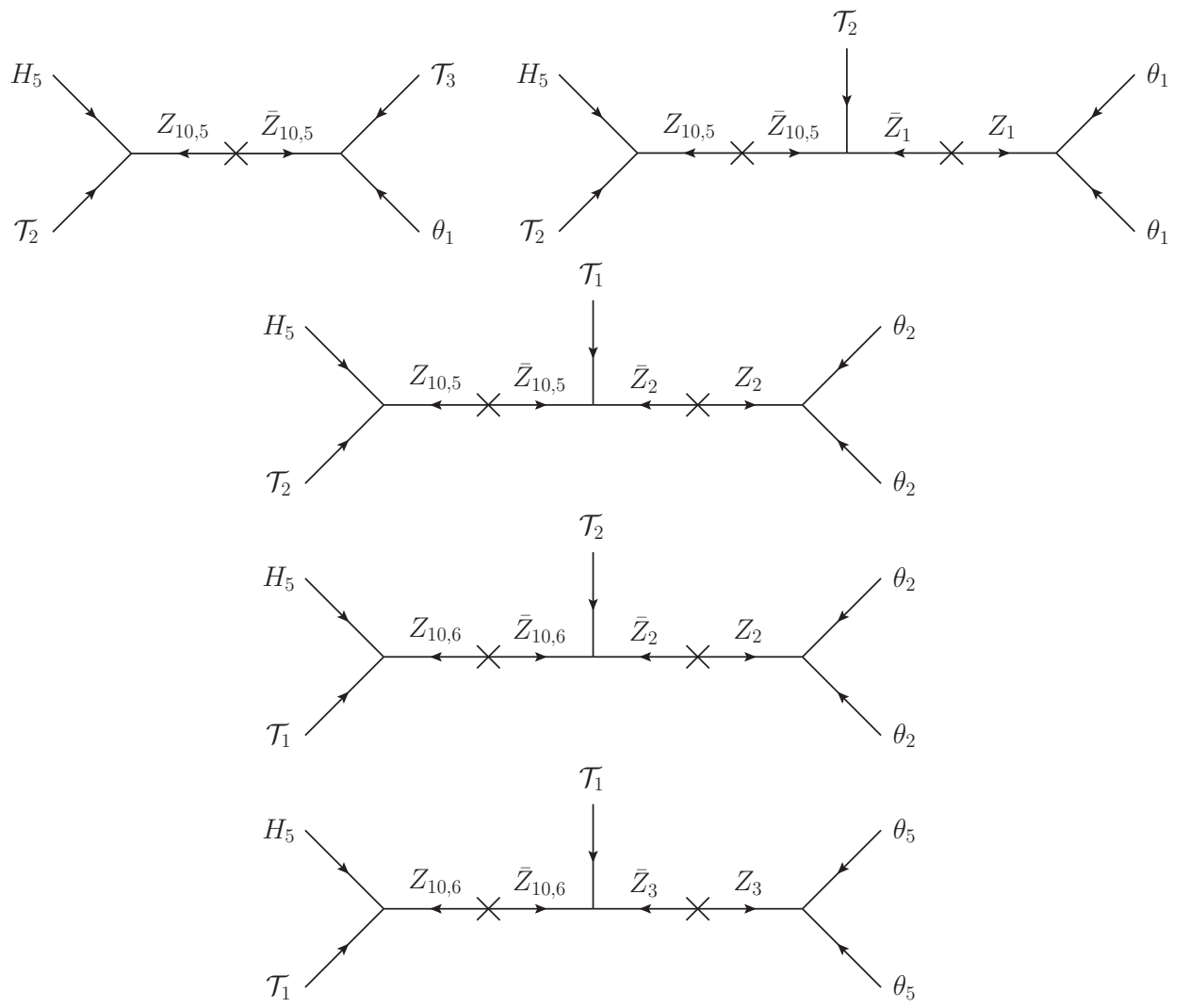

Figure 8. Supergraphs leading to the effective superpotential $W_{u}$ of eq. (4.16) when the heavy messenger fields get integrated out.

\begin{tabular}{|cccccccccc|}
\hline & $\mathrm{SU}(5)$ & $\mathbb{Z}_{2}$ & $\mathbb{Z}_{3}$ & $\mathbb{Z}_{4}$ & $\mathbb{Z}_{5}$ & $\mathbb{Z}_{6}$ & $\mathbb{Z}_{7}$ & $\mathbb{Z}_{7}$ & $\mathbb{Z}_{2}$ \\
\hline$H_{5}$ & $\mathbf{5}$ &. &. &. &. &. &. &. &. \\
$\bar{H}_{5}$ & $\overline{\mathbf{5}}$ &. & 2 &. & 1 & 4 & 6 & 6 &. \\
$\mathcal{T}_{1}$ & $\mathbf{1 0}$ &. &. &. &. &. & 6 & 5 & 1 \\
$\mathcal{T}_{2}$ & $\mathbf{1 0}$ &. &. &. &. &. & 1 &. & 1 \\
$\mathcal{T}_{3}$ & $\mathbf{1 0}$ &. &. &. &. &. &. &. & 1 \\
$\mathcal{F}_{1}$ & $\overline{\mathbf{5}}$ &. &. &. & 3 & 2 & 5 & 6 & 1 \\
$\mathcal{F}_{2}$ & $\overline{\mathbf{5}}$ &. & 2 &. & 3 &. & 2 & 3 & 1 \\
$\mathcal{F}_{3}$ & $\overline{\mathbf{5}}$ &. & 1 & 3 & 4 &. & 1 & 1 & 1 \\
\hline
\end{tabular}

Table 6. SU(5) representations and charges under discrete shaping symmetries of the MSSM fields and colour triplets of the model presented in subsection 4.2. A dot denotes charge zero.

We continue now by discussing additional details concerning the use of the DMPM in this model. Like in the previous model, Planck-scale suppressed operators generate mass terms for the five-dimensional Higgs representations

$$
W_{5}^{\mathrm{eff}}=\mu H_{5} \bar{H}_{5}+\mu^{\prime} H_{5}^{\prime} \bar{H}_{5}^{\prime},
$$




\begin{tabular}{|cccccccccc|}
\hline & $\mathrm{SU}(5)$ & $\mathbb{Z}_{2}$ & $\mathbb{Z}_{3}$ & $\mathbb{Z}_{4}$ & $\mathbb{Z}_{5}$ & $\mathbb{Z}_{6}$ & $\mathbb{Z}_{7}$ & $\mathbb{Z}_{7}$ & $\mathbb{Z}_{2}$ \\
\hline$H_{24}$ & $\mathbf{2 4}$ &. &. &. &. & 4 &. &. &. \\
$H_{24}^{\prime}$ & $\mathbf{2 4}$ &. &. &. &. & 2 &. &. &. \\
$S$ & $\mathbf{1}$ & 1 & 2 &. & 2 &. &. &. &. \\
$S^{\prime}$ & $\mathbf{1}$ &. &. & 3 &. &. &. &. &. \\
$\theta_{1}$ & $\mathbf{1}$ &. &. &. &. &. & 6 &. &. \\
$\theta_{2}$ & $\mathbf{1}$ &. &. &. &. &. &. & 1 &. \\
$\theta_{3}$ & $\mathbf{1}$ &. & 1 & 2 & 1 & 2 & 2 & 2 &. \\
$\theta_{4}$ & $\mathbf{1}$ &. & 2 & 2 & 1 &. & 5 & 5 &. \\
$\theta_{5}$ & $\mathbf{1}$ &. &. &. &. & 3 & 1 & 2 &. \\
\hline
\end{tabular}

Table 7. SU(5) representations and charges under discrete shaping symmetries of the superfields obtaining VEVs at around the GUT scale of the model presented in subsection 4.2. A dot denotes charge zero.

\begin{tabular}{|cccccccccc|}
\hline & $\mathrm{SU}(5)$ & $\mathbb{Z}_{2}$ & $\mathbb{Z}_{3}$ & $\mathbb{Z}_{4}$ & $\mathbb{Z}_{5}$ & $\mathbb{Z}_{6}$ & $\mathbb{Z}_{7}$ & $\mathbb{Z}_{7}$ & $\mathbb{Z}_{2}$ \\
\hline$H_{5}^{\prime}$ & $\mathbf{5}$ & 1 & 1 &. &. & 4 & 1 & 1 &. \\
$\bar{H}_{5}^{\prime}$ & $\overline{\mathbf{5}}$ & 1 &. &. & 1 & 2 &. &. &. \\
$X_{45}$ & $\mathbf{4 5}$ &. & 1 &. & 4 & 4 & 1 & 1 &. \\
$\bar{X}_{45}$ & $\overline{\mathbf{4 5}}$ & 1 &. &. & 4 & 2 & 6 & 6 &. \\
$Y_{45}$ & $\mathbf{4 5}$ &. & 2 &. & 3 & 2 & 1 & 1 &. \\
$\bar{Y}_{45}$ & $\overline{\mathbf{4 5}}$ & 1 & 2 &. &. & 4 & 6 & 6 &. \\
$Z_{50}$ & $\mathbf{5 0}$ & 1 &. &. & 1 &. & 1 & 1 &. \\
$\bar{Z}_{50}$ & $\overline{\mathbf{5 0}}$ &. & 1 &. & 2 &. & 6 & 6 &. \\
$X_{45}^{\prime}$ & $\mathbf{4 5}$ & 1 &. &. & 4 &. &. &. &. \\
$\bar{X}_{45}^{\prime}$ & $\overline{\mathbf{4 5}}$ &. & 1 &. & 4 &. &. &. &. \\
$Y_{45}^{\prime}$ & $\mathbf{4 5}$ & 1 & 1 &. & 3 & 4 &. &. &. \\
$\bar{Y}_{45}^{\prime}$ & $\overline{\mathbf{4 5}}$ &. &. &. &. & 2 &. &. &. \\
$Z_{50}^{\prime}$ & $\mathbf{5 0}$ &. & 2 &. & 1 & 2 &. &. &. \\
$\bar{Z}_{50}^{\prime}$ & $\overline{\mathbf{5 0}}$ & 1 & 2 &. & 2 & 4 &. &. &. \\
\hline
\end{tabular}

Table 8. SU(5) representations and charges under discrete shaping symmetries of the fields in the DMPM sector of the model presented in subsection 4.2. A dot denotes charge zero.

where $\mu=\left\langle\theta_{3}\right\rangle^{4} / M_{\mathrm{Pl}}^{3}$ and $\mu^{\prime}=\left\langle\theta_{4}\right\rangle^{4} / M_{\mathrm{Pl}}^{3}$. Note that although they appear at the same order, a modest hierarchy $\left\langle\theta_{3}\right\rangle<\left\langle\theta_{4}\right\rangle$, as we have in our model, will sufficiently split their masses. Furthermore, after integrating out the 45-dimensional messengers of the DMPM, the mass matrices for doublet and triplet components of the 5- and 50-dimensional 


\begin{tabular}{|cccccccccc|}
\hline & $\mathrm{SU}(5)$ & $\mathbb{Z}_{2}$ & $\mathbb{Z}_{3}$ & $\mathbb{Z}_{4}$ & $\mathbb{Z}_{5}$ & $\mathbb{Z}_{6}$ & $\mathbb{Z}_{7}$ & $\mathbb{Z}_{7}$ & $\mathbb{Z}_{2}$ \\
\hline$Z_{5}$ & $\mathbf{5}$ &. & 2 & 1 & 1 & 4 & 6 & 6 & 1 \\
$\bar{Z}_{5}$ & $\overline{\mathbf{5}}$ &. & 1 &. & 4 & 2 & 1 & 1 & 1 \\
$Z_{10,1}$ & $\mathbf{1 0}$ &. & 1 &. & 1 &. & 3 & 2 & 1 \\
$\bar{Z}_{10,1}$ & $\overline{\mathbf{1 0}}$ &. & 2 & 1 & 4 &. & 4 & 5 & 1 \\
$Z_{10,2}$ & $\mathbf{1 0}$ &. &. & 1 &. & 4 & 1 &. & 1 \\
$\bar{Z}_{10,2}$ & $\overline{\mathbf{1 0}}$ &. &. &. &. & 2 & 6 &. & 1 \\
$Z_{10,3}$ & $\mathbf{1 0}$ &. & 2 &. & 1 & 2 & 6 & 5 & 1 \\
$\bar{Z}_{10,3}$ & $\overline{\mathbf{1 0}}$ &. & 1 & 1 & 4 & 4 & 1 & 2 & 1 \\
$Z_{10,4}$ & $\mathbf{1 0}$ &. &. & 1 &. & 2 & 1 &. & 1 \\
$\bar{Z}_{10,4}$ & $\overline{\mathbf{1 0}}$ &. &. &. &. & 4 & 6 &. & 1 \\
$Z_{45,1}$ & $\mathbf{4 5}$ &. & 1 &. & 2 & 2 & 5 & 4 & 1 \\
$\bar{Z}_{45,1}$ & $\overline{\mathbf{4 5}}$ & 1 &. &. & 1 & 4 & 2 & 3 & 1 \\
\hline$Z_{10,5}$ & $\mathbf{1 0}$ &. &. &. &. &. & 6 &. & 1 \\
$Z_{10,6}$ & $\mathbf{1 0}$ &. &. &. &. &. & 1 & 2 & 1 \\
$Z_{1}$ & $\mathbf{1}$ &. &. &. &. &. & 2 &. &. \\
$Z_{2}$ & $\mathbf{1}$ &. &. &. &. &. &. & 5 &. \\
$Z_{3}$ & $\mathbf{1}$ &. &. &. &. &. & 5 & 3 &. \\
\hline
\end{tabular}

Table 9. SU(5) representations and charges under discrete shaping symmetries of the flavon and flavour messenger fields of the model presented in subsection 4.2 . Note that the messengers $Z_{5,1} \bar{Z}_{5,1}$, $Z_{10,1} \bar{Z}_{10,1}, Z_{10,2} \bar{Z}_{10,2}, Z_{10,3} \bar{Z}_{10,3}, Z_{10,4} \bar{Z}_{10,4}$ and $Z_{45,1} \bar{Z}_{45,1}$ have no direct mass term, but get their masses through VEVs of $S$ and $S^{\prime}$. The other messenger fields have direct mass terms, so their corresponding barred field is not shown in the table. A dot denotes charge zero.

superfields are given by

$$
m_{D}=\left(\begin{array}{cc}
\mu & 0 \\
0 & \mu^{\prime}
\end{array}\right), \quad m_{T}=\left(\begin{array}{cccc}
\mu & 0 & 0 & -\frac{V_{1}^{2}}{\langle S\rangle} \\
0 & \mu^{\prime} & -\frac{V_{1}^{2}}{\langle S\rangle} & 0 \\
-\frac{V_{1}^{2}}{\langle S\rangle} & 0 & \langle S\rangle & 0 \\
0 & -\frac{V_{1}^{2}}{\langle S\rangle} & 0 & \langle S\rangle
\end{array}\right),
$$

where $V_{1}$ is defined in eq. (3.11). After the heavy 50-dimensional fields get integrated out the mass matrices become

$$
m_{D}=\left(\begin{array}{cc}
\mu & 0 \\
0 & \mu^{\prime}
\end{array}\right), \quad m_{T}=\left(\begin{array}{cc}
\mu & -\frac{V_{1}^{4}}{\langle S\rangle^{3}} \\
-\frac{V_{1}^{4}}{\langle S\rangle^{3}} & \mu^{\prime}
\end{array}\right) .
$$

This leads to an effective triplet mass for dimension five proton decay of

$$
M_{T}^{\operatorname{dim}=5}=\left(m_{T}^{-1}\right)_{11}^{-1}=\mu-\frac{V_{1}^{8}}{\langle S\rangle^{6} \mu^{\prime}} \approx-\frac{V_{1}^{8} M_{\mathrm{Pl}}^{3}}{\langle S\rangle^{6}\left\langle\theta_{4}\right\rangle^{4}},
$$


and the effective triplet masses suppressing dimension six proton decay are given by

$$
\left(M_{T}^{\operatorname{dim}=6}\right)^{2}=\left(M_{\bar{T}}^{\operatorname{dim}=6}\right)^{2}=\left(m_{T}^{-1} m_{T}^{\dagger-1}\right)_{11}^{-1} \approx \frac{\left|V_{1}\right|^{8}}{|\langle S\rangle|^{6}} .
$$

As for the first example model we can estimate the values of the relevant masses in an explicit example from the known Yukawa couplings. In a small angle approximation the down-type Yukawa couplings are given by

$$
y_{d} \sim \frac{\left\langle H_{24}\right\rangle^{2}}{\langle S\rangle^{2}} \frac{\left\langle H_{24}\right\rangle\left\langle\theta_{3}\right\rangle}{\left\langle S^{\prime}\right\rangle^{2}} \frac{1}{y_{s}} \approx 1.6 \cdot 10^{-4} \quad \text { with } \quad y_{s} \sim \frac{\left\langle H_{24}^{\prime}\right\rangle\left\langle\theta_{4}\right\rangle}{\left\langle S^{\prime}\right\rangle^{2}} \approx 3 \cdot 10^{-3}
$$

and

$$
y_{b} \sim \frac{\left\langle H_{24}^{\prime}\right\rangle}{\left\langle S^{\prime}\right\rangle} \approx 0.18
$$

where the numerical values for the Yukawa couplings taken from [25] are valid for $\tan \beta=30$ and order one coefficients have been neglected. For the effective triplet masses of dimension five and six proton decay, respectively, the $\mu$-term and the mass of the additional, heavier Higgs doublets the following estimates emerge

$$
M_{T}^{\operatorname{dim}=5} \approx 1.4 \cdot 10^{18}, \mathrm{GeV}, \quad M_{T}^{\operatorname{dim}=6} \approx 10^{12} \mathrm{GeV}, \quad \mu \approx 7 \mathrm{TeV}, \quad \mu^{\prime} \approx 800 \mathrm{TeV} .
$$

The parameters of superpotential (b) from section 3.1.2 have been chosen to be real with values $\tan \beta_{V}=0.5, \lambda=10^{-4}$ and $M=2.4 \cdot 10^{12} \mathrm{GeV}$. The mass of the 45-dimensional and 50-dimensional superfields has been set to $\langle S\rangle=10^{18} \mathrm{GeV}$. With the numbers of eq. (4.26) we find

$$
M_{\mathrm{SUSY}} \approx 24 \mathrm{TeV}, \quad M_{\mathrm{GUT}} \approx 4 \cdot 10^{17} \mathrm{GeV} .
$$

Thus, also in this model, proton decay can be suppressed by large effective triplet masses. DTS is achieved through the DMPM and the light doublet gets its mass from a Planckscale suppressed operator. The Yukawa sector of the model features viable fermion masses and quark mixing angles.

\section{Proton decay}

We have split our discussion of proton decay into two parts. In the first part we comment on proton decay induced by dimension five operators in the superpotential. These operators are usually considered to be more dangerous for the validity of any SUSY GUT model. We will argue why we are more predictive than ordinary models and still should be able to evade current experimental bounds. In the second part we will comment on dimension six proton decay operators, which appear also in non-SUSY GUTs. The dimension six proton decay operator emerging from the exchange of heavy gauge bosons are considered to be not as dangerous in SUSY GUTs due to the usually higher unification scale [6]. The dimension six proton decay mediated by colour triplets, however, has to be suppressed by a high enough effective triplet mass, as for the case of dimension five proton decay. 


\subsection{Proton decay from dimension five operators}

We will adopt for this section a notation similar to the SLHA convention [36], for more details see appendix A. The superpotential describing the couplings of the matter fields to the heavy colour triplet reads

$$
\begin{aligned}
W_{T}= & \epsilon_{\alpha \beta}\left(-\frac{1}{2}\left(Y_{q q}\right)_{i j} \epsilon_{a b c} T^{a} Q_{i}^{\alpha b} Q_{j}^{\beta c}+\left(Y_{q l}\right)_{i j} \bar{T}^{a} Q_{i}^{\alpha a} L_{j}^{\beta}\right) \\
& +\left(Y_{u e}\right)_{i j} T^{a} \bar{U}_{i}^{a} \bar{E}_{j}-\left(Y_{u d}\right)_{i j} \epsilon_{a b c} \bar{T}^{a} \bar{U}_{i}^{b} \bar{D}_{j}^{c}+M_{T} T^{a} \bar{T}^{a} .
\end{aligned}
$$

In many $\mathrm{SU}(5)$ models at least for the first two generations it was assumed that the Yukawa couplings are generated or significantly corrected by some set of higher-dimensional operators, see for instance, $[24,37,38]$. But, usually, there is no control over which operator is the dominant one and hence it is not possible to calculate how strong exactly the heavy triplets couple to the MSSM fields. In our setup this is not the case. For every entry of the Yukawa matrix we have specified the operator with only very small corrections, if any. Therefore we know how strong the MSSM fields couple to the heavy triplets if we know the MSSM Yukawa couplings at the GUT scale. To be more precise the new Yukawa couplings are related to the MSSM ones only via CG coefficients which are fixed by the gauge structure of the underlying operator. We find for our first example model

$$
\begin{aligned}
Y_{d} & =\operatorname{Diag}\left(y_{d}, y_{s}, y_{b}\right), & Y_{e} & =\operatorname{Diag}\left(-\frac{1}{2} y_{d}, 6 y_{s},-\frac{3}{2} y_{b}\right), \\
Y_{q l} & =\operatorname{Diag}\left(y_{d}, y_{s},-\frac{3}{2} y_{b}\right), & Y_{u d} & =\operatorname{Diag}\left(\frac{2}{3} y_{d},-4 y_{s}, y_{b}\right), \\
Y_{q q} & =Y_{u}, & Y_{u e} & =Y_{u},
\end{aligned}
$$

where the structure of $Y_{u}$ can be read off from eq. (4.2). For the second model we find

$$
\begin{aligned}
& Y_{d}=\left(\begin{array}{ccc}
0 & y_{d, 12} & 0 \\
y_{d, 21} & y_{s} & 0 \\
0 & 0 & y_{b}
\end{array}\right), \\
& Y_{e}^{T}=\left(\begin{array}{ccc}
0 & -\frac{1}{2} y_{d, 12} & 0 \\
6 y_{d, 21} & 6 y_{s} & 0 \\
0 & 0 & -\frac{3}{2} y_{b}
\end{array}\right), \\
& Y_{q l}=\left(\begin{array}{ccc}
0 & y_{d, 12} & 0 \\
y_{d, 21} & y_{s} & 0 \\
0 & 0 & -\frac{3}{2} y_{b}
\end{array}\right), \\
& Y_{u d}=\left(\begin{array}{ccc}
0 & \frac{2}{3} y_{d, 12} & 0 \\
-4 y_{d, 21} & -4 y_{s} & 0 \\
0 & 0 & y_{b}
\end{array}\right) \text {, } \\
& Y_{q q}=Y_{u}, \\
& Y_{u e}=Y_{u} \text {, }
\end{aligned}
$$

where the structure of $Y_{u}$ can be read off from eq. (4.15). ${ }^{10}$

The dimension five operators which violate baryon number after integrating out the triplets read

$$
W_{\not B}=\frac{1}{M_{T}^{\mathrm{eff}}}\left[\frac{1}{2} Y_{q q}^{i j} Y_{q l}^{m n} Q_{i} Q_{j} Q_{m} L_{n}+Y_{u e}^{i j} Y_{u d}^{m n} \bar{U}_{i} \bar{E}_{j} \bar{U}_{m} \bar{D}_{n}\right]
$$

\footnotetext{
${ }^{10} \mathrm{GUT}$ textures for proton decay, without fully constructed models, have been considered, for example, in $[38,39]$.
} 
where we have suppressed $\epsilon$-tensors. The first operator is called the $L L L L$ operator and the second one the $R R R R$ operator. To make definite predictions for the proton decay rate one would have to take the RGE evolution of these operators to low energies into account and dress the operators with a closed loop including MSSM particles to calculate the decay rate of the proton, see e.g. [19] and references therein. This goes clearly beyond the scope of this paper. Before we give some more qualitative statements about what we expect for the proton decay rate in comparison to other models we want to argue first that the operators in eq. (5.8) together with $M_{T}^{\text {eff }}$ from eq. (2.18) give the dominant dimension five contribution.

Inside the additional higher dimensional representations used in the DMPM there are additional colour triplets which could in principle give the same operators like in eq. (5.8) but with a weaker suppression. We have explicitly checked that the DMPM by itself is safe: the additional colour triplets from 5-, 45- and 50-dimensional representations can only mediate operators that are suppressed compared to the leading contribution by one or more powers of $H_{24} / M_{\mathrm{Pl}}$ and $H_{24}^{\prime} / M_{\mathrm{Pl}}$.

We have also checked for each of our models that no other Yukawa matrix entries (through components in the messengers) lead to a lower $M_{T}^{\text {dim }=5}$. This is, of course, model dependent and has to be checked for any specific model.

We now turn to a qualitative discussion of dimension five proton decay in the considered class of models. Firstly, we want to point out that especially in the first model one could expect some decay modes of the proton to be suppressed because $Y_{q l}$ and $Y_{u d}$ are diagonal in flavour space. Therefore, decays which need a flavour transition in these matrices would be suppressed. This reinforces the statement that our setup is more predictive than conventional ones, due to the better control over the flavour structure of all the Yukawa matrices governing proton decay.

Secondly, introducing a second adjoint in a renormalizable way gives us enough freedom to enhance the effective triplet mass $M_{T}^{\text {dim }=5}$ to comfortable levels, cf. section 3 . In the case of superpotential (b), we get a mass of roughly $5 \cdot 10^{16} \mathrm{GeV}$ for a SUSY scale of $1 \mathrm{TeV}$ which increases with increasing $M_{\mathrm{SUSY}}$, see section 3.1.2. For superpotential (a) the situation is more involved and depends on the specific GUT scale, and the effective triplet mass can be easily above $10^{18} \mathrm{GeV}$.

To conclude the discussion of the dimension five operators mediating proton decay we also want to stress that in our setup we need only a moderate value of $\tan \beta \approx 25$. As it was pointed out by Lucas and Raby [40] especially the contribution from the $R R R R$ operator is enhanced by $\tan ^{2} \beta$ for large $\tan \beta$ which poses a challenge for many GUT scenarios which rely on $\tan \beta \approx 50$. Our setup with $y_{\tau} / y_{b}=\frac{3}{2}$ and $\tan \beta \approx 25$ has therefore a suppression of proton decay via the $R R R R$ operator by a factor of four compared to these models.

In summary, together with the large effective triplet mass in the double missing partner mechanism we hence expect the proton decay rate to be sufficiently small but possibly in the reach of the next generation of proton decay experiments. 


\subsection{Proton decay from dimension six operators}

We now turn to the discussion of dimension six proton decay. As discussed in section 2, the dimension six proton decay mediated by colour triplets originates from the Kähler potential

$$
K_{T}=T^{a} T^{\dagger a}+\bar{T}^{a} \bar{T}^{\dagger a} .
$$

When the colour triplets are integrated out, the dimension six baryon number violating Kähler operators emerge as

$$
K_{B^{\prime}}=-\frac{1}{\left(M_{\bar{T}}^{\operatorname{dim}=6}\right)^{2}} \frac{1}{2} Y_{q q}^{i j} Y_{u e}^{* m n} Q_{i} Q_{j} \bar{U}_{m}^{\dagger} \bar{E}_{n}^{\dagger}-\frac{1}{\left(M_{T}^{\mathrm{dim}=6}\right)^{2}} Y_{q l}^{i j} Y_{u d}^{* m n} Q_{i} L_{j} \bar{U}_{m}^{\dagger} \bar{D}_{n}^{\dagger}+\text { h.c. },
$$

where the Yukawa coupling matrices are defined by $W_{T}$ of eq. (5.8). As for the dimension five proton decay, these operators need to be subject to a RGE evolution from the GUT scale to the proton mass scale. Note however, that these operators do not need to be dressed with superparticles and therefore the proton decay rates obtained from dimension six operators are independent of the details of the SUSY spectrum.

For completeness, we will now briefly discuss as well proton decay from exchange of heavy gauge bosons. There is no substantial difference in our models compared to other SUSY SU(5) models since the gauge structure is exactly the same.

To make the discussion a little bit more analogous to the previous discussion we define an effective GUT scale

$$
M_{\mathrm{GUT}}^{\mathrm{eff}}=\frac{M_{\mathrm{GUT}}}{\sqrt{\alpha_{u}}},
$$

where $\alpha_{u}$ is the unified gauge coupling at the GUT scale and $M_{\mathrm{GUT}} \equiv M_{V}$ is the mass of the leptoquark vector bosons, cf. the discussion in section 3. Just integrating out the heavy gauge bosons in the tree-level diagrams governing proton decay, and from dimensional analysis, we can estimate the lifetime of the proton to be

$$
\Gamma_{p} \approx \alpha_{u}^{2} \frac{m_{p}^{5}}{M_{V}^{4}}=\frac{m_{p}^{5}}{\left(M_{\mathrm{GUT}}^{\mathrm{eff}}\right)^{4}},
$$

where $m_{p}$ is the proton mass and we have neglected RGE effects and order one coefficients from nuclear matrix elements and such. The most stringent bound on the proton lifetime is $\tau\left(p \rightarrow \pi^{0} e^{+}\right)>8.2 \cdot 10^{33}$ years [10] which yields an effective GUT scale of about $M_{\mathrm{GUT}}^{\text {eff }} \gtrsim$ $2 \cdot 10^{16} \mathrm{GeV}$. In section 3 we have seen that the GUT scale can be easily above $10^{16} \mathrm{GeV}$ and $\sqrt{\alpha_{u}} \approx 1 / 5$ such that the proton decay rate from dimension six operators is sufficiently small but possibly in the reach of the next generation of proton decay experiments.

\section{Summary and conclusions}

In this work we have discussed how the double missing partner mechanism solution to the doublet-triplet splitting problem in four-dimensional supersymmetric SU(5) Grand Unified Theories can be combined with predictive models featuring novel predictions for the quarklepton Yukawa coupling ratios at the GUT scale. 
We have argued that towards this goal a second SU(5) breaking Higgs field in the adjoint representation is very useful. We systematically discussed all possible renormalizable superpotentials with two adjoint Higgs fields, also calculating the corresponding constraints on the GUT scale and effective triplet mass from a two-loop gauge coupling unification analysis. We found that the effective masses of the colour triplet, which enter dimension five and six proton decay, can easily be raised enough to avoid problems with proton decay (more than feasible with standard non-renormalizable Higgs potentials with only one adjoint GUT Higgs field).

We have constructed two explicit flavour models with different predictions for the GUT scale Yukawa sector. A set of shaping symmetries and a renormalizable messenger sector for the models is presented, which guarantees that only the desired effective GUT operators are generated when the heavy degrees of freedom are integrated out. In addition, we also include all possible effective Planck-scale suppressed operators consistent with our symmetries, and make sure that they do not spoil our results. The models stay perturbative until close to the Planck scale, such that our predictions do not suffer from large uncertainties due to these Planck-scale suppressed operators. They serve as existence proofs that predictive models for the GUT scale quark-lepton mass relations can be combined successfully with the DMPM solution for solving the DTS problem.

We also provide several useful appendices for GUT flavour model building: for instance, one appendix contains the Clebsch-Gordan coefficients for the couplings of the colour triplets, which are required for calculating the rates for proton decay induced by their exchange. We provide detailed tables with the Clebsch-Gordan coefficients for the possible dimension five and six GUT Yukawa operators. We also discuss there how one can use GUT Higgs potentials for flavour model building, where a (discrete) R-symmetry is broken spontaneously around the GUT scale. R-symmetries are a helpful ingredient of many flavour models, especially when they include non-Abelian family symmetries.

In summary, we have demonstrated that four-dimensional supersymmetric SU(5) GUTs with successful doublet-triplet splitting can be combined with predictive models featuring promising predictions for the quark-lepton Yukawa coupling ratios at the GUT scale. We have provided the tools for the construction of even more ambitious GUT models of flavour with additional non-Abelian family symmetries, as well as towards the calculation of the predictions for the rates of the various nucleon decay channels in such models by systematically providing the required Clebsch-Gordan coefficients.

\section{Acknowledgments}

This work is supported by the Swiss National Science Foundation. We thank Borut Bajc and David Emmanuel-Costa for useful discussions.

\section{A Yukawa coupling ratios including colour triplets}

The MSSM superpotential is given by

$$
W=\epsilon_{\alpha \beta}\left(\left(Y_{e}\right)^{i j} H_{d}^{\alpha} L_{i}^{\beta} \bar{E}_{j}+\left(Y_{d}\right)^{i j} H_{d}^{\alpha} Q_{i}^{\beta a} \bar{D}_{j}^{a}+\left(Y_{u}\right)^{i j} H_{u}^{\beta} Q_{i}^{\alpha a} \bar{U}_{j}^{a}+\mu H_{u}^{\alpha} H_{d}^{\beta}\right),
$$


where $i, j$ are generation indices, $\epsilon_{\alpha \beta}$ the Levi-Civita tensor $\left(\epsilon_{12}=1\right), \alpha, \beta$ are $\mathrm{SU}(2)$ indices and $a, b$ and $c$ are $\mathrm{SU}(3)$ indices. ${ }^{11}$ Adding a pair of colour triplets $T$ and $\bar{T}$, we get the additional terms

$$
\begin{aligned}
W_{T}= & \epsilon_{\alpha \beta}\left(-\frac{1}{2}\left(Y_{q q}\right)_{i j} \epsilon_{a b c} T^{a} Q_{i}^{\alpha b} Q_{j}^{\beta c}+\left(Y_{q l}\right)_{i j} \bar{T}^{a} Q_{i}^{\alpha a} L_{j}^{\beta}\right) \\
& +\left(Y_{u e}\right)_{i j} T^{a} \bar{U}_{i}^{a} \bar{E}_{j}-\left(Y_{u d}\right)_{i j} \epsilon_{a b c} \bar{T}^{a} \bar{U}_{i}^{b} \bar{D}_{j}^{c}+M_{T} T^{a} \bar{T}^{a}
\end{aligned}
$$

where $\epsilon_{a b c}$ is the three indices Levi-Civita tensor (with $\epsilon_{123}=1$ ).

Extending the SM gauge group to SU(5), we embed the MSSM superfields in a 5-plet $H_{5}, \overline{5}$-plets $\bar{H}_{5}$ and $\mathcal{F}_{i}$, and 10 -plets $\mathcal{T}_{i}$ as in

$$
\begin{aligned}
H_{5} & =\left(\begin{array}{lll}
T^{r} & T^{g} T^{b} H_{u}^{+} H_{u}^{0}
\end{array}\right), \\
\bar{H}_{5} & =\left(\begin{array}{llll}
\bar{T}^{r} & \bar{T}^{g} \bar{T}^{b} H_{d}^{-}-H_{d}^{0}
\end{array}\right), \\
\mathcal{F}_{i} & =\left(\begin{array}{lllll}
\bar{D}_{i}^{r} \bar{D}_{i}^{g} \bar{D}_{i}^{b} & E_{i} & -\nu_{i}
\end{array}\right), \\
\mathcal{T}_{i} & =\frac{1}{\sqrt{2}}\left(\begin{array}{ccccc}
0 & -\bar{U}_{i}^{b} & \bar{U}_{i}^{g} & -U_{i}^{r} & -D_{i}^{r} \\
\bar{U}_{i}^{b} & 0 & -\bar{U}_{i}^{r} & -U_{i}^{g} & -D_{i}^{g} \\
-\bar{U}_{i}^{g} & \bar{U}_{i}^{r} & 0 & -U_{i}^{b} & -D_{i}^{b} \\
U_{i}^{r} & U_{i}^{g} & U_{i}^{b} & 0 & -\bar{E}_{i} \\
D_{i}^{r} & D_{i}^{g} & D_{i}^{b} & \bar{E}_{i} & 0
\end{array}\right),
\end{aligned}
$$

where $r, g, b$ are the $\mathrm{SU}(3)$ colours and $U, D$ and $\nu, E$ are the components of $\mathrm{SU}(2)$-doublets $Q$ and $L .{ }^{12}$ We can write down the renormalizable superpotential terms

$$
W=\left(Y_{T F}\right)_{i j} \mathcal{T}_{i}^{a b}\left(\mathcal{F}_{j}\right)_{a}\left(\bar{H}_{5}\right)_{b}+\frac{1}{2}\left(Y_{T T}\right)_{i j} \epsilon_{a b c d e} \mathcal{T}_{i}^{a b} \mathcal{T}_{j}^{c d} H_{5}^{e}+\mu_{5} H_{5}^{a}\left(\bar{H}_{5}\right)_{a}
$$

where now $a, b, c, d, e$ are $\mathrm{SU}(5)$-indices and $\epsilon_{a b c d e}$ is the respective Levi-Civita tensor. From the embedding of the MSSM fields, one obtains the minimal SU(5) GUT scale relations

$$
\begin{aligned}
\mu & =M_{T}=\mu_{5}, \\
Y_{d} & =Y_{e}^{T}=Y_{q l}=Y_{u d}=\frac{1}{\sqrt{2}} Y_{T F}, \\
Y_{u} & =Y_{u}^{T}=Y_{q q}=Y_{u e}=2 Y_{T T} .
\end{aligned}
$$

The relation $Y_{d}=Y_{e}^{T}$ is highly disfavoured as was already discussed in section 2.4. The conventional approach is to add a 45-dimensional Higgs representation which generates a relative factor of -3 between the Yukawa couplings of the charged leptons and downtype quarks [3]. In this work we instead focus on an approach where the ratios between Yukawa couplings are fixed by the CG coefficients of higher-dimensional operators where in addition an adjoint Higgs representation of SU(5) is added [11, 22]. This approach was

\footnotetext{
${ }^{11}$ This definition coincides with the definitions of [36].

${ }^{12}$ Likewise $H_{d}=\left(\begin{array}{ll}H_{d}^{0} & H_{d}^{-}\end{array}\right)^{T}$ and $H_{u}=\left(\begin{array}{ll}H_{u}^{+} & H_{u}^{0}\end{array}\right)^{T}$.
} 


\begin{tabular}{|c|c|c|c|c|c|c|c|}
\hline & $A B$ & $C D$ & $R$ & $\left(Y_{d}\right)_{i j}$ & $:\left(Y_{e}\right)_{j i}$ & $:\left(Y_{q l}\right)_{i j}$ & $:\left(Y_{u d}\right)_{i j}$ \\
\hline \multirow{5}{*}{$\rightarrow$} & \multicolumn{2}{|c|}{$\mathcal{F}_{j} \mathcal{T}_{i} \bar{H}_{5}$} & - & 1 & 1 & 1 & 1 \\
\hline & $H_{24} \mathcal{T}_{i}$ & $\mathcal{F}_{j} \bar{H}_{5}$ & 10 & 1 & $: \quad 6$ & $:$ & $: \quad-4$ \\
\hline & $H_{24} \mathcal{T}_{i}$ & $\mathcal{F}_{j} \bar{H}_{5}$ & 15 & 1 & $: 0$ & $: \quad-1$ & 0 \\
\hline & $H_{24} \bar{H}_{5}$ & $\mathcal{F}_{j} \mathcal{T}_{i}$ & $\overline{5}$ & 1 & $:$ & $: \quad-\frac{2}{3}$ & $: \quad-\frac{2}{3}$ \\
\hline & $H_{24} \bar{H}_{5}$ & $\mathcal{F}_{j} \mathcal{T}_{i}$ & $\overline{45}$ & 1 & $: \quad-3$ & $: \quad-2$ & 2 \\
\hline \multirow[t]{2}{*}{$\rightarrow$} & $H_{24} \mathcal{F}_{j}$ & $\mathcal{T}_{i} \bar{H}_{5}$ & $\overline{5}$ & 1 & $: \quad-\frac{3}{2}$ & $: \quad-$ & 1 \\
\hline & $H_{24} \mathcal{F}_{j}$ & $\mathcal{T}_{i} \bar{H}_{5}$ & $\overline{45}$ & 1 & $: \quad \frac{3}{2}$ & $: \quad-\frac{1}{2}$ & $: \quad-1$ \\
\hline & \multicolumn{2}{|c|}{$\mathcal{F}_{j} \mathcal{T}_{i} \bar{H}_{45}$} & - & 1 & $: \quad-3$ & $: \quad \sqrt{3}$ & $:-\sqrt{3}$ \\
\hline & $H_{24} \mathcal{T}_{i}$ & $\mathcal{F}_{j} \bar{H}_{45}$ & 10 & 1 & $:-18$ & $: \quad \sqrt{3}$ & $: 4 \sqrt{3}$ \\
\hline & $H_{24} \mathcal{T}_{i}$ & $\mathcal{F}_{j} \bar{H}_{45}$ & 40 & 1 & $: 0$ & & $:-\frac{\sqrt{3}}{2}$ \\
\hline & $H_{24} \mathcal{T}_{i}$ & $\mathcal{F}_{j} \bar{H}_{45}$ & 175 & 1 & $: \quad \frac{36}{23}$ & & $:-\frac{16 \sqrt{3}}{23}$ \\
\hline & $H_{24} \bar{H}_{45}$ & $\mathcal{F}_{j} \mathcal{T}_{i}$ & $\overline{5}$ & 1 & $: \quad 1$ & $:-\frac{2}{\sqrt{3}}$ & $:-\frac{2}{\sqrt{3}}$ \\
\hline & $H_{24} \bar{H}_{45}$ & $\mathcal{F}_{j} \mathcal{T}_{i}$ & $\overline{45}$ & 1 & $: \quad-3$ & $: \quad x$ & $:-x$ \\
\hline & $H_{24} \mathcal{F}_{j}$ & $\mathcal{T}_{i} \bar{H}_{45}$ & $\overline{5}$ & 1 & & $:-\frac{3 \sqrt{3}}{2}$ & $:-\sqrt{3}$ \\
\hline & $H_{24} \mathcal{F}_{j}$ & $\mathcal{T}_{i} \bar{H}_{45}$ & $\overline{45}$ & 1 & $: \quad-\frac{1}{2}$ & $:-\frac{\sqrt{3}}{2}$ & $:-\frac{1}{\sqrt{3}}$ \\
\hline & $H_{24} \mathcal{F}_{j}$ & $\mathcal{T}_{i} \bar{H}_{45}$ & $\overline{70}$ & 1 & $: \quad \frac{9}{4}$ & $:-\frac{3 \sqrt{3}}{4}$ & $:-\sqrt{3}$ \\
\hline
\end{tabular}

Table 10. $Y_{T F}$-like CG ratios for the dimension 4 operator and effective dimension 5 operators $W \supset(A B)_{R}(C D)_{\bar{R}}$ (involving 5- and 45-dimensional Higgs fields) corresponding to the the left diagram in figure 9 . Note that one combination has a free parameter $x$ due to the ambiguity of the index contraction. See main text for more details.
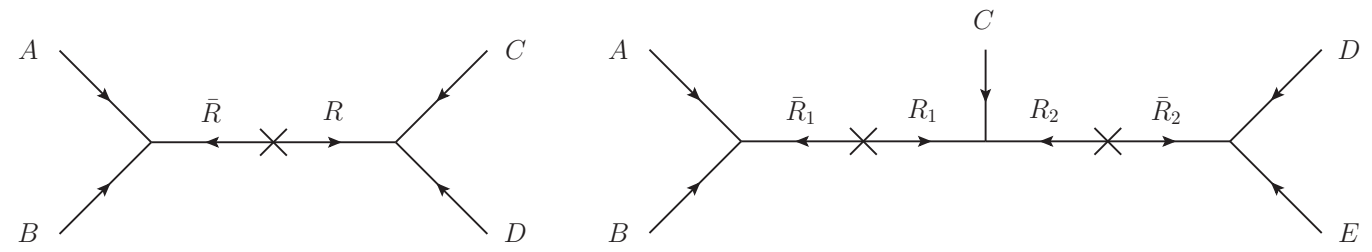

Figure 9. Supergraphs generating Yukawa couplings upon integrating out messengers fields in representation $R, \bar{R}$, etc.

briefly reviewed in section 2.4 and for a thorough discussion we refer the interested reader to the original papers, see also [41].

In this appendix we will extend the previous discussions to include also the relative CG coefficents to the triplets. In $[11,22]$ only $Y_{d}, Y_{e}$ and $Y_{u}$ were discussed while here we will also discuss in detail the implications of this approach for $Y_{q l}, Y_{u d}, Y_{q q}, Y_{u e}$. The list of the resulting ratios for dimension 4 and 5 operators with Higgs fields in a 5 - and 45-dimensional representation can be found in tables 10 and 11, where the labels for the representations is defined by figure 9. The corresponding results for dimension six operators are given in tables $12-15$. The ones used in the example models are marked with a " $\rightarrow$ " in the tables. 


\begin{tabular}{|ccccccccccccc|}
\hline$A B$ & $C D$ & $R$ & $\left(Y_{u}\right)_{i j}:$ & $\left(Y_{u}\right)_{j i}:$ & $\left(Y_{q q}\right)_{i j}$ & $:$ & $\left(Y_{u e}\right)_{i j}:$ & $\left(Y_{u e}\right)_{j i}$ \\
\hline$\rightarrow$ & \multicolumn{2}{c}{$\mathcal{T}_{i} \mathcal{T}_{j} H_{5}$} & - & 1 & $:$ & 1 & $:$ & 1 & $:$ & 1 & $:$ & 1 \\
$H_{24} H_{5}$ & $\mathcal{T}_{i} \mathcal{T}_{j}$ & 5 & 1 & $:$ & 1 & $:$ & $-\frac{2}{3}$ & $:$ & $-\frac{2}{3}$ & $:$ & $-\frac{2}{3}$ \\
$H_{24} H_{5}$ & $\mathcal{T}_{i} \mathcal{T}_{j}$ & 45 & 1 & $:$ & -1 & $:$ & 0 & $:$ & -2 & $:$ & 2 \\
$H_{24} \mathcal{T}_{i}$ & $\mathcal{T}_{j} H_{5}$ & 10 & 1 & $:$ & -4 & $:$ & 1 & $:$ & -4 & $:$ & 6 \\
$H_{24} \mathcal{T}_{i}$ & $\mathcal{T}_{j} H_{5}$ & 40 & 1 & $:$ & $\frac{1}{2}$ & $:$ & $-\frac{1}{2}$ & $:$ & -1 & $:$ & 0 \\
\hline $\mathcal{T}_{i} \mathcal{T}_{j} H_{45}$ & - & 1 & $:$ & -1 & $:$ & 0 & $:$ & $\sqrt{3}$ & $:$ & $-\sqrt{3}$ \\
$H_{24} \mathcal{T}_{i}$ & $\mathcal{T}_{j} H_{45}$ & 10 & 1 & $:$ & 4 & $:$ & 0 & $:$ & $-4 \sqrt{3}$ & $:$ & $-6 \sqrt{3}$ \\
$H_{24} \mathcal{T}_{i}$ & $\mathcal{T}_{j} H_{45}$ & 15 & 1 & $:$ & 0 & $:$ & $-\frac{\sqrt{3}}{2}$ & $:$ & 0 & $:$ & 0 \\
$H_{24} \mathcal{T}_{i}$ & $\mathcal{T}_{j} H_{45}$ & 40 & 1 & $:$ & $-\frac{7}{2}$ & $:$ & $\frac{3 \sqrt{3}}{2}$ & $:$ & $-\sqrt{3}$ & $:$ & 0 \\
$H_{24} \mathcal{T}_{i}$ & $\mathcal{T}_{j} H_{45}$ & 175 & 1 & $:$ & $\frac{16}{19}$ & $:$ & $-\frac{21 \sqrt{3}}{38}$ & $:$ & $-\frac{16 \sqrt{3}}{19}$ & $:$ & $-\frac{12 \sqrt{3}}{19}$ \\
$H_{24} H_{45}$ & $\mathcal{T}_{i} \mathcal{T}_{j}$ & 5 & 1 & $:$ & 1 & $:$ & $-\frac{2}{\sqrt{3}}$ & $:$ & $-\frac{2}{\sqrt{3}}$ & $:$ & $-\frac{2}{\sqrt{3}}$ \\
$H_{24} H_{45}$ & $\mathcal{T}_{i} \mathcal{T}_{j}$ & 45 & 1 & $:$ & -1 & $:$ & 0 & $:$ & $x$ & $:$ & $-x$ \\
$H_{24} H_{45}$ & $\mathcal{T}_{i} \mathcal{T}_{j}$ & 50 & 0 & $:$ & 0 & $:$ & 1 & $:$ & -2 & $:$ & -2 \\
\hline
\end{tabular}

Table 11. $Y_{T T}$-like CG ratios for the dimension 4 operator and effective dimension 5 operators $W \supset(A B)_{R}(C D)_{\bar{R}}$ (involving 5- and 45-dimensional Higgs fields) corresponding to the the left diagram in figure 9 . Note that one combination has a free parameter $x$ due to the ambiguity of the index contraction. See main text for more details.

There are a few comments in order. First, note that several topologies involving a 45dimensional messenger field exhibit a free parameter, simply because the tensor product $45 \otimes 24$ contains two 45-dimensional representations. Hence, there are two operators possibly giving two different ratios so that any ratio is possible depending on the coefficients of the two operators. For these cases we write $x$ in the tables.

We want to mention as well that, unlike at the renormalizable level, the up-type quark Yukawa and related matrices do not have to be symmetric or antisymmetric. Consider, for example, the operator $\left(H_{24} \mathcal{T}_{1}\right)_{\mathbf{1 0}}\left(H_{5} \mathcal{T}_{2}\right)_{\overline{\mathbf{1 0}}}$. Due to the symmetries and messenger content the operator $\left(H_{24} \mathcal{T}_{2}\right)_{\mathbf{1 0}}\left(H_{5} \mathcal{T}_{1}\right)_{\overline{\mathbf{1 0}}}$ could be forbidden. In this case we find $\left(Y_{u}\right)_{12} /\left(Y_{u}\right)_{21}=$ -4 . Hence we have adopted the following notation for the ratios in the tables for the Yukawa couplings related to $Y_{u}$

$$
\left(Y_{u}\right)_{i j}:\left(Y_{u}\right)_{j i}:\left(Y_{q q}\right)_{i j}:\left(Y_{u e}\right)_{i j}:\left(Y_{u e}\right)_{j i}=a: b: c: d: e
$$

which reduces for the diagonal entries of the Yukawa matrices to

$$
\left(Y_{u}\right)_{i i}:\left(Y_{q q}\right)_{i i}:\left(Y_{u e}\right)_{i i}=(a+b): c:(d+e) .
$$

The ratios related to $Y_{d}$ do not have this extra complication since none of them could be expected to be symmetric or anti-symmetric in the first place. 


\begin{tabular}{|c|c|c|c|c|c|c|c|c|c|c|c|}
\hline & $A B$ & $C$ & $D E$ & $R_{1}, R_{2}$ & $\left(Y_{d}\right)_{i j}$ & . & $\left(Y_{e}\right)_{j i}$ & & $\left(Y_{q l}\right)_{i j}$ & : & $\left(Y_{u d}\right)_{i j}$ \\
\hline & $\mathcal{T}_{i} \bar{H}_{5}$ & $\mathcal{F}_{j}$ & $H_{24} H_{24}$ & 5,1 & 1 & : & 1 & & 1 & : & 1 \\
\hline & $\mathcal{T}_{i} \bar{H}_{5}$ & $\mathcal{F}_{j}$ & $H_{24} H_{24}$ & 5,24 & 1 & : & $-\frac{3}{2}$ & : & $-\frac{3}{2}$ & : & 1 \\
\hline & $\mathcal{T}_{i} \bar{H}_{5}$ & $\mathcal{F}_{j}$ & $H_{24} H_{24}$ & 45,24 & 1 & : & $\frac{3}{2}$ & & $-\frac{1}{2}$ & : & -1 \\
\hline & $\mathcal{T}_{i} \bar{H}_{5}$ & $\mathcal{F}_{j}$ & $H_{24} H_{24}$ & 45,75 & 1 & : & -3 & : & 1 & $:$ & -1 \\
\hline & $H_{24} \bar{H}_{5}$ & $H_{24}$ & $\mathcal{F}_{j} \mathcal{T}_{i}$ & $\overline{5}, 5$ & 1 & : & 1 & : & $\frac{4}{9}$ & $:$ & $\frac{4}{9}$ \\
\hline & $H_{24} \bar{H}_{5}$ & $H_{24}$ & $\mathcal{F}_{j} \mathcal{T}_{i}$ & $\overline{5}, 45$ & 1 & : & -3 & : & $\frac{4}{3}$ & $:$ & $-\frac{4}{3}$ \\
\hline & $H_{24} \bar{H}_{5}$ & $H_{24}$ & $\mathcal{F}_{j} \mathcal{T}_{i}$ & $\overline{45}, 5$ & 1 & : & 1 & & $\frac{4}{3}$ & . & $\frac{4}{3}$ \\
\hline & $H_{24} \bar{H}_{5}$ & $H_{24}$ & $\mathcal{F}_{j} \mathcal{T}_{i}$ & $\overline{45}, 45$ & 1 & : & -3 & : & $x$ & $:$ & $-x$ \\
\hline & $H_{24} \bar{H}_{5}$ & $H_{24}$ & $\mathcal{F}_{j} \mathcal{T}_{i}$ & $\overline{70}, 5$ & 1 & : & 1 & . & $\frac{8}{9}$ & $:$ & $\frac{8}{9}$ \\
\hline & $H_{24} \bar{H}_{5}$ & $H_{24}$ & $\mathcal{F}_{j} \mathcal{T}_{i}$ & $\overline{70}, 45$ & 1 & : & -3 & & $\frac{8}{3}$ & $:$ & $-\frac{8}{3}$ \\
\hline & $\mathcal{F}_{j} \mathcal{T}_{i}$ & $\bar{H}_{5}$ & $H_{24} H_{24}$ & 5,1 & 1 & : & 1 & : & 1 & : & 1 \\
\hline & $\mathcal{F}_{j} \mathcal{T}_{i}$ & $\bar{H}_{5}$ & $H_{24} H_{24}$ & 5,24 & 1 & : & 1 & . & $-\frac{2}{3}$ & : & $-\frac{2}{3}$ \\
\hline & $\mathcal{F}_{j} \mathcal{T}_{i}$ & $\bar{H}_{5}$ & $H_{24} H_{24}$ & 45,24 & 1 & : & -3 & : & -2 & $:$ & 2 \\
\hline & $\mathcal{F}_{j} \mathcal{T}_{i}$ & $\bar{H}_{5}$ & $H_{24} H_{24}$ & 45,75 & 1 & : & -3 & & 1 & $:$ & -1 \\
\hline & $H_{24} \bar{H}_{5}$ & $\mathcal{T}_{i}$ & $H_{24} \mathcal{F}_{j}$ & $\overline{5}, \overline{5}$ & 1 & : & $-\frac{3}{2}$ & & 1 & $:$ & $-\frac{2}{3}$ \\
\hline & $H_{24} \bar{H}_{5}$ & $\mathcal{T}_{i}$ & $H_{24} \mathcal{F}_{j}$ & $\overline{5}, \overline{45}$ & 1 & : & $\frac{3}{2}$ & & $\frac{1}{3}$ & $:$ & $\frac{2}{3}$ \\
\hline & $H_{24} \bar{H}_{5}$ & $\mathcal{T}_{i}$ & $H_{24} \mathcal{F}_{j}$ & $\overline{45}, \overline{5}$ & 1 & : & $\frac{9}{2}$ & & 3 & $:$ & 2 \\
\hline$\rightarrow$ & $H_{24} \bar{H}_{5}$ & $\mathcal{T}_{i}$ & $H_{24} \mathcal{F}_{j}$ & $\overline{45}, \overline{45}$ & 1 & : & $-\frac{1}{2}$ & : & 1 & $:$ & $\frac{2}{3}$ \\
\hline & $H_{24} \bar{H}_{5}$ & $\mathcal{T}_{i}$ & $H_{24} \mathcal{F}_{j}$ & $\overline{45}, \overline{70}$ & 1 & : & $\frac{9}{4}$ & & $\frac{3}{2}$ & $:$ & 2 \\
\hline & $H_{24} \bar{H}_{5}$ & $\mathcal{T}_{i}$ & $H_{24} \mathcal{F}_{j}$ & $\overline{70}, \overline{45}$ & 1 & : & $\frac{3}{2}$ & & $\frac{2}{3}$ & $:$ & $\frac{4}{3}$ \\
\hline & $H_{24} \bar{H}_{5}$ & $\mathcal{T}_{i}$ & $H_{24} \mathcal{F}_{j}$ & $\overline{70}, \overline{70}$ & 1 & : & $\frac{3}{4}$ & • & 1 & $:$ & $\frac{2}{3}$ \\
\hline & $\mathcal{F}_{j} \bar{H}_{5}$ & $H_{24}$ & $H_{24} \mathcal{T}_{i}$ & $\overline{10}, 10$ & 1 & : & 36 & : & 1 & $:$ & 16 \\
\hline & $\mathcal{F}_{j} \bar{H}_{5}$ & $H_{24}$ & $H_{24} \mathcal{T}_{i}$ & $\overline{10}, 15$ & 1 & : & 0 & & 1 & $:$ & 0 \\
\hline & $\mathcal{F}_{j} \bar{H}_{5}$ & $H_{24}$ & $H_{24} \mathcal{T}_{i}$ & $\overline{10}, 40$ & 1 & : & 0 & & 1 & $:$ & 1 \\
\hline & $\mathcal{F}_{j} \bar{H}_{5}$ & $H_{24}$ & $H_{24} \mathcal{T}_{i}$ & $\overline{10}, 175$ & 1 & : & $\frac{72}{61}$ & & 1 & $:$ & $\frac{64}{61}$ \\
\hline & $\mathcal{F}_{j} \bar{H}_{5}$ & $H_{24}$ & $H_{24} \mathcal{T}_{i}$ & $\overline{15}, 10$ & 1 & ${ }^{\circ}$ & 0 & 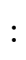 & -1 & $:$ & 0 \\
\hline & $\mathcal{F}_{j} \bar{H}_{5}$ & $H_{24}$ & $H_{24} \mathcal{T}_{i}$ & $\overline{15}, 15$ & 1 & : & 0 & ${ }^{\circ}$ & -1 & $:$ & 0 \\
\hline & $\mathcal{F}_{j} \bar{H}_{5}$ & $H_{24}$ & $H_{24} \mathcal{T}_{i}$ & $\overline{15}, 175$ & 1 & : & 0 & : & -1 & $:$ & 0 \\
\hline
\end{tabular}

Table 12. $Y_{T F^{-}}$-like $\mathrm{CG}$ ratios for the effective dimension 6 operators $W \supset(A B)_{R_{1}} C(D E)_{R_{2}}$ corresponding to the the right diagram in figure 9. Note one combination has a free parameter $x$. See main text for more details.

If the considered model contains Higgs fields in 5- and 45-dimensional representations, ${ }^{13}$ there are two Higgs doublet pairs in the spectrum and care should be taken that unification

\footnotetext{
${ }^{13}$ One could also imagine using 45-dimensional Higgs fields exclusively. However, this severely exacerbates the doublet-triplet splitting problem as now one has to split the doublets from even more component fields that can generate proton decay operators.
} 


\begin{tabular}{|c|c|c|c|c|c|c|c|c|}
\hline$A B$ & $C$ & $D E$ & $R_{1}, R_{2}$ & $\left(Y_{d}\right)_{i j}$ & $:\left(Y_{e}\right)_{j i}$ & $:\left(Y_{q l}\right)_{i \jmath}$ & j: & $\left(Y_{u d}\right)_{i j}$ \\
\hline$H_{24} \mathcal{F}_{j}$ & $H_{24}$ & $\mathcal{T}_{i} \bar{H}_{5}$ & $\overline{5}, 5$ & 1 & $\frac{9}{4}$ & $\frac{9}{4}$ & $:$ & 1 \\
\hline$H_{24} \mathcal{F}_{j}$ & $H_{24}$ & $\mathcal{T}_{i} \bar{H}_{5}$ & $\overline{5}, 45$ & 1 & $: \quad-\frac{9}{4}$ & $\frac{3}{4}$ & : & -1 \\
\hline$H_{24} \mathcal{F}_{j}$ & $H_{24}$ & $\mathcal{T}_{i} \bar{H}_{5}$ & $\overline{45}, 5$ & 1 & $\frac{3}{4}$ & $\frac{3}{4}$ & $:$ & 1 \\
\hline$H_{24} \mathcal{F}_{j}$ & $H_{24}$ & $\mathcal{T}_{i} \bar{H}_{5}$ & $\overline{45}, 45$ & 1 & $x$ & $: \quad-\frac{x}{3}$ & $:$ & -1 \\
\hline$H_{24} \mathcal{F}_{j}$ & $H_{24}$ & $\mathcal{T}_{i} \bar{H}_{5}$ & $\overline{70}, 5$ & 1 & $\frac{9}{8}$ & $\frac{9}{8}$ & $:$ & 1 \\
\hline$H_{24} \mathcal{F}_{j}$ & $H_{24}$ & $\mathcal{T}_{i} \bar{H}_{5}$ & $\overline{70}, 45$ & 1 & $: \quad-\frac{9}{8}$ & $\frac{3}{8}$ & : & -1 \\
\hline$H_{24} \mathcal{F}_{j}$ & $\bar{H}_{5}$ & $H_{24} \mathcal{T}_{i}$ & $\overline{5}, 10$ & 1 & $: \quad-9$ & $: \quad-\frac{3}{2}$ & : & -4 \\
\hline$H_{24} \mathcal{F}_{j}$ & $\bar{H}_{5}$ & $H_{24} \mathcal{T}_{i}$ & $\overline{5}, 15$ & 1 & 0 & $\frac{3}{2}$ & : & 0 \\
\hline$H_{24} \mathcal{F}_{j}$ & $\bar{H}_{5}$ & $H_{24} \mathcal{T}_{i}$ & $\overline{45}, 10$ & 1 & 9 & $: \quad-\frac{1}{2}$ & : & 4 \\
\hline$H_{24} \mathcal{F}_{j}$ & $\bar{H}_{5}$ & $H_{24} \mathcal{T}_{i}$ & $\overline{45}, 40$ & 1 & 0 & 1 & : & 1 \\
\hline$H_{24} \mathcal{F}_{j}$ & $\bar{H}_{5}$ & $H_{24} \mathcal{T}_{i}$ & $\overline{45}, 175$ & 1 & $: \quad \frac{18}{19}$ & $\frac{23}{38}$ & : & $\frac{16}{19}$ \\
\hline$H_{24} \mathcal{F}_{j}$ & $\bar{H}_{5}$ & $H_{24} \mathcal{T}_{i}$ & $\overline{70}, 15$ & 1 & 0 & $\frac{3}{4}$ & $:$ & 0 \\
\hline$H_{24} \mathcal{F}_{j}$ & $\bar{H}_{5}$ & $H_{24} \mathcal{T}_{i}$ & $\overline{70}, 175$ & 1 & $\frac{9}{7}$ & $\frac{33}{28}$ & $:$ & $\frac{8}{7}$ \\
\hline$H_{24} \bar{H}_{5}$ & $\mathcal{F}_{j}$ & $H_{24} \mathcal{T}_{i}$ & $\overline{5}, 10$ & 1 & 6 & $: \quad-\frac{2}{3}$ & $:$ & $\frac{8}{3}$ \\
\hline$H_{24} \bar{H}_{5}$ & $\mathcal{F}_{j}$ & $H_{24} \mathcal{T}_{i}$ & $\overline{5}, 15$ & 1 & 0 & $: \quad \frac{2}{3}$ & : & 0 \\
\hline$H_{24} \bar{H}_{5}$ & $\mathcal{F}_{j}$ & $H_{24} \mathcal{T}_{i}$ & $\overline{45}, 10$ & 1 & $:-18$ & $: \quad-2$ & $:$ & -8 \\
\hline$H_{24} \bar{H}_{5}$ & $\mathcal{F}_{j}$ & $H_{24} \mathcal{T}_{i}$ & $\overline{45}, 40$ & 1 & 0 & 1 & $:$ & 1 \\
\hline$H_{24} \bar{H}_{5}$ & $\mathcal{F}_{j}$ & $H_{24} \mathcal{T}_{i}$ & $\overline{45}, 175$ & 1 & $\frac{36}{23}$ & $\frac{38}{23}$ & $:$ & $\frac{32}{23}$ \\
\hline$H_{24} \bar{H}_{5}$ & $\mathcal{F}_{j}$ & $H_{24} \mathcal{T}_{i}$ & $\overline{70}, 15$ & 1 & 0 & $: \quad \frac{4}{3}$ & $:$ & 0 \\
\hline$H_{24} \bar{H}_{5}$ & $\mathcal{F}_{j}$ & $H_{24} \mathcal{T}_{i}$ & $\overline{70}, 175$ & 1 & $\frac{12}{11}$ & $\frac{28}{33}$ & $:$ & $\frac{32}{33}$ \\
\hline $\mathcal{F}_{j} \bar{H}_{5}$ & $\mathcal{T}_{i}$ & $H_{24} H_{24}$ & $\overline{10}, 1$ & 1 & 1 & 1 & $:$ & 1 \\
\hline $\mathcal{F}_{j} \bar{H}_{5}$ & $\mathcal{T}_{i}$ & $H_{24} H_{24}$ & $\overline{10}, 24$ & 1 & 6 & 1 & : & -4 \\
\hline $\mathcal{F}_{j} \bar{H}_{5}$ & $\mathcal{T}_{i}$ & $H_{24} H_{24}$ & $\overline{10}, 75$ & 1 & $: \quad-3$ & 1 & $:$ & -1 \\
\hline $\mathcal{F}_{j} \bar{H}_{5}$ & $\mathcal{T}_{i}$ & $H_{24} H_{24}$ & $\overline{15}, 24$ & 1 & 0 & $: \quad-1$ & : & 0 \\
\hline
\end{tabular}

Table 13. Continuation of table 12: $Y_{T F}$-like CG ratios for the effective dimension 6 operators $W \supset$ $(A B)_{R_{1}} C(D E)_{R_{2}}$ corresponding to the the right diagram in figure 9. Note another combination with a free parameter $x$. See main text for more details.

is still possible. One solution is mixing both and making one linear combination heavy while one stays at the electroweak scale. The simplest term generating such a mixing is

$$
W \supset H_{24} H_{5} \bar{H}_{45} \propto H_{u} H_{d}^{\overline{45}}-\frac{2}{\sqrt{3}} T \bar{T}^{\overline{45}},
$$

where we suppressed any additional MSSM multiplets in $\bar{H}_{45}$. Since a $\overline{\mathbf{4 5}}$ contains more potentially dangerous MSSM multiplets, it is natural to have the heavy linear combination be predominantly in the $\overline{\mathbf{4 5}}$. Then it is possible to treat $\bar{H}_{45}$ like a messenger field and the renormalizable operator $\mathcal{F} \mathcal{T} \bar{H}_{45}$ turns into the non-renormalizable operator $(\mathcal{F} \mathcal{T})_{45}\left(H_{24} \bar{H}_{5}\right)_{\overline{45}}$, cf. table 10. Analogous limits can be deduced trivially. If the approxi- 


\begin{tabular}{|ccccccccccccc|}
\hline$A B$ & $C$ & $D E$ & $R_{1}, R_{2}$ & $\left(Y_{u}\right)_{i j}:$ & $\left(Y_{u}\right)_{j i}:$ & $\left(Y_{q q}\right)_{i j}:$ & $\left(Y_{u e}\right)_{i j}:$ & $\left(Y_{u e}\right)_{j i}$ \\
\hline$H_{24} \mathcal{T}_{i}$ & $H_{5}$ & $H_{24} \mathcal{T}_{j}$ & 10,10 & 1 & $:$ & 1 & $:$ & $-\frac{1}{4}$ & $:$ & 6 & $:$ & 6 \\
$H_{24} \mathcal{T}_{i}$ & $H_{5}$ & $H_{24} \mathcal{T}_{j}$ & 10,40 & 1 & $:$ & -8 & $:$ & -1 & $:$ & 0 & $:$ & -12 \\
$H_{24} \mathcal{T}_{i}$ & $H_{5}$ & $H_{24} \mathcal{T}_{j}$ & 15,40 & 1 & $:$ & 0 & $:$ & 1 & $:$ & 0 & $:$ & 0 \\
$H_{24} \mathcal{T}_{i}$ & $H_{5}$ & $H_{24} \mathcal{T}_{j}$ & 40,10 & 1 & $:$ & $-\frac{1}{8}$ & $:$ & $\frac{1}{8}$ & $:$ & $\frac{3}{2}$ & $:$ & 0 \\
$H_{24} \mathcal{T}_{i}$ & $H_{5}$ & $H_{24} \mathcal{T}_{j}$ & 40,15 & 0 & $:$ & 1 & $:$ & 1 & $:$ & 0 & $:$ & 0 \\
$H_{24} \mathcal{T}_{i}$ & $H_{5}$ & $H_{24} \mathcal{T}_{j}$ & 40,175 & 1 & $:$ & $\frac{23}{32}$ & $:$ & $\frac{19}{32}$ & $:$ & $\frac{3}{4}$ & $:$ & 0 \\
$H_{24} \mathcal{T}_{i}$ & $H_{5}$ & $H_{24} \mathcal{T}_{j}$ & 175,40 & 1 & $:$ & $\frac{32}{23}$ & $:$ & $\frac{19}{23}$ & $:$ & 0 & $:$ & $\frac{24}{23}$ \\
$H_{24} \mathcal{T}_{i}$ & $H_{5}$ & $H_{24} \mathcal{T}_{j}$ & 175,175 & 1 & $:$ & 1 & $:$ & $\frac{41}{40}$ & $:$ & $\frac{6}{5}$ & $:$ & $\frac{6}{5}$ \\
$\mathcal{T}_{i} \mathcal{T}_{j}$ & $H_{5}$ & $H_{24} H_{24}$ & $\overline{5}, 1$ & 1 & $:$ & 1 & $:$ & 1 & $:$ & 1 & $:$ & 1 \\
$\mathcal{T}_{i} \mathcal{T}_{j}$ & $H_{5}$ & $H_{24} H_{24}$ & $\overline{5}, 24$ & 1 & $:$ & 1 & $:$ & $-\frac{2}{3}$ & $:$ & $-\frac{2}{3}$ & $:$ & $-\frac{2}{3}$ \\
$\mathcal{T}_{i} \mathcal{T}_{j}$ & $H_{5}$ & $H_{24} H_{24}$ & $\overline{45}, 24$ & 1 & $:$ & -1 & $:$ & 0 & $:$ & -2 & $:$ & 2 \\
$\mathcal{T}_{i} \mathcal{T}_{j}$ & $H_{5}$ & $H_{24} H_{24}$ & $\overline{45}, 75$ & 1 & $:$ & -1 & $:$ & 0 & $:$ & 1 & $:$ & -1 \\
$\mathcal{T}_{i} \mathcal{T}_{j}$ & $H_{5}$ & $H_{24} H_{24}$ & $\overline{50}, 75$ & 0 & $:$ & 0 & $:$ & 1 & $:$ & -2 & $:$ & -2 \\
$\mathcal{T}_{i} H_{5}$ & $\mathcal{T}_{j}$ & $H_{24} H_{24}$ & $\overline{10}, 1$ & 1 & $:$ & 1 & $:$ & 1 & $:$ & 1 & $:$ & 1 \\
$\mathcal{T}_{i} H_{5}$ & $\mathcal{T}_{j}$ & $H_{24} H_{24}$ & $\overline{10}, 24$ & 1 & $:$ & $-\frac{1}{4}$ & $:$ & $-\frac{1}{4}$ & $:$ & $-\frac{3}{2}$ & $:$ & 1 \\
$\mathcal{T}_{i} H_{5}$ & $\mathcal{T}_{j}$ & $H_{24} H_{24}$ & $\overline{10}, 75$ & 1 & $:$ & -1 & $:$ & -1 & $:$ & 3 & $:$ & 1 \\
$\mathcal{T}_{i} H_{5}$ & $\mathcal{T}_{j}$ & $H_{24} H_{24}$ & $\overline{40}, 24$ & 1 & $:$ & 2 & $:$ & -1 & $:$ & 0 & $:$ & -2 \\
$\mathcal{T}_{i} H_{5}$ & $\mathcal{T}_{j}$ & $H_{24} H_{24}$ & $\overline{40}, 75$ & 1 & $:$ & -1 & $:$ & $\frac{1}{2}$ & $:$ & 0 & $:$ & -2 \\
\hline
\end{tabular}

Table 14. $Y_{T T}$-like $\mathrm{CG}$ ratios for the effective dimension 6 operators $W \supset(A B)_{R_{1}} C(D E)_{R_{2}}$ corresponding to the the right diagram in figure 9 .

mation $m_{45} \gg\left\langle H_{24}\right\rangle$ does not hold, one has to take into account the full mass matrix for the Higgs doublets including the term in eq. (A.13).

\section{B Two-loop RGEs in extensions to the MSSM}

The renormalization group equations for gauge couplings at two-loop are given by [42, 43]

$$
\mu \frac{d}{d \mu} g_{a}=\frac{g_{a}^{3}}{16 \pi^{2}} b_{a}+\frac{g_{a}^{3}}{\left(16 \pi^{2}\right)^{2}}\left(\sum_{b=1}^{3} B_{a b} g_{b}^{2}-\sum_{f} C_{a}^{f} \operatorname{tr}\left(Y_{f}^{\dagger} Y_{f}\right)\right)
$$

in the $\overline{\mathrm{DR}}$ renormalization scheme, where $\mu$ is the renormalization scale and $f$ runs over all Yukawa coupling matrices. In the MSSM, the beta function coefficients are given by (in GUT normalisation for $g_{1}$ )

$$
b_{a}=\left(\begin{array}{c}
\frac{33}{5} \\
1 \\
-3
\end{array}\right), \quad B_{a b}=\left(\begin{array}{ccc}
\frac{199}{25} & \frac{27}{5} & \frac{88}{5} \\
\frac{9}{5} & 25 & 24 \\
\frac{11}{5} & 9 & 14
\end{array}\right)
$$




\begin{tabular}{|c|c|c|c|c|c|c|c|c|c|c|c|}
\hline$A B$ & $C$ & $D E$ & $R_{1}, R_{2}$ & $\left(Y_{u}\right)_{i j}$ & : & $\left(Y_{u}\right)_{j}$ & $:\left(Y_{q q}\right)_{i j}$ & : & $\left(Y_{u e}\right)_{i j}$ & $j:$ & $\left(Y_{u e}\right)_{j i}$ \\
\hline$H_{24} \mathcal{T}_{i}$ & $\mathcal{T}_{j}$ & $H_{24} H_{5}$ & 10,5 & 1 & : & -4 & $-\frac{2}{3}$ & : & $\frac{8}{3}$ & : & -4 \\
\hline$H_{24} \mathcal{T}_{i}$ & $\mathcal{T}_{j}$ & $H_{24} H_{5}$ & 10,45 & 1 & $:$ & 4 & 0 & $:$ & 8 & $:$ & 12 \\
\hline$H_{24} \mathcal{T}_{i}$ & $\mathcal{T}_{j}$ & $H_{24} H_{5}$ & 15,45 & 1 & $:$ & 0 & 1 & $:$ & 0 & : & 0 \\
\hline$H_{24} \mathcal{T}_{i}$ & $\mathcal{T}_{j}$ & $H_{24} H_{5}$ & 40,5 & 1 & $:$ & $\frac{1}{2}$ & $\frac{1}{3}$ & 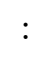 & $\frac{2}{3}$ & : & 0 \\
\hline$H_{24} \mathcal{T}_{i}$ & $\mathcal{T}_{j}$ & $H_{24} H_{5}$ & 40,45 & 1 & : & $-\frac{7}{2}$ & -3 & : & 2 & : & 0 \\
\hline$H_{24} \mathcal{T}_{i}$ & $\mathcal{T}_{j}$ & $H_{24} H_{5}$ & 40,70 & 1 & : & $\frac{1}{2}$ & $\frac{2}{3}$ & : & $\frac{4}{3}$ & : & 0 \\
\hline$H_{24} \mathcal{T}_{i}$ & $\mathcal{T}_{j}$ & $H_{24} H_{5}$ & 175,45 & 1 & : & $\frac{16}{19}$ & $\frac{21}{19}$ & : & $\frac{32}{19}$ & : & $\frac{24}{19}$ \\
\hline$H_{24} \mathcal{T}_{i}$ & $\mathcal{T}_{j}$ & $H_{24} H_{5}$ & 175,70 & 1 & $:$ & $\frac{8}{7}$ & $\frac{20}{21}$ & $:$ & $\frac{16}{21}$ & : & $\frac{8}{7}$ \\
\hline $\mathcal{T}_{i} \mathcal{T}_{j}$ & $H_{24}$ & $H_{24} H_{5}$ & $\overline{5}, 5$ & 1 & : & 1 & $\frac{4}{9}$ & : & $\frac{4}{9}$ & : & $\frac{4}{9}$ \\
\hline $\mathcal{T}_{i} \mathcal{T}_{j}$ & $H_{24}$ & $H_{24} H_{5}$ & $\overline{5}, 45$ & 1 & : & 1 & $\frac{4}{3}$ & : & $\frac{4}{3}$ & : & $\frac{4}{3}$ \\
\hline $\mathcal{T}_{i} \mathcal{T}_{j}$ & $H_{24}$ & $H_{24} H_{5}$ & $\overline{5}, 70$ & 1 & : & 1 & $\frac{8}{9}$ & : & $\frac{8}{9}$ & : & $\frac{8}{9}$ \\
\hline $\mathcal{T}_{i} \mathcal{T}_{j}$ & $H_{24}$ & $H_{24} H_{5}$ & $\overline{45}, 5$ & 1 & $:$ & -1 & 0 & $:$ & $\frac{4}{3}$ & : & $-\frac{4}{3}$ \\
\hline $\mathcal{T}_{i} \mathcal{T}_{j}$ & $H_{24}$ & $H_{24} H_{5}$ & $\overline{45}, 45$ & 1 & : & -1 & 0 & : & $x$ & : & $-x$ \\
\hline $\mathcal{T}_{i} \mathcal{T}_{j}$ & $H_{24}$ & $H_{24} H_{5}$ & $\overline{45}, 70$ & 1 & : & -1 & 0 & . & $\frac{8}{3}$ & : & $-\frac{8}{3}$ \\
\hline $\mathcal{T}_{i} \mathcal{T}_{j}$ & $H_{24}$ & $H_{24} H_{5}$ & $\overline{50}, 45$ & 0 & : & 0 & 1 & : & -2 & : & -2 \\
\hline$H_{24} \mathcal{T}_{i}$ & $H_{24}$ & $\mathcal{T}_{j} H_{5}$ & $10, \overline{10}$ & 1 & : & 16 & 1 & : & 16 & : & 36 \\
\hline$H_{24} \mathcal{T}_{i}$ & $H_{24}$ & $\mathcal{T}_{j} H_{5}$ & $10, \overline{40}$ & 1 & : & -2 & $: \quad-\frac{1}{2}$ & : & 4 & : & 0 \\
\hline$H_{24} \mathcal{T}_{i}$ & $H_{24}$ & $\mathcal{T}_{j} H_{5}$ & $15, \overline{10}$ & 1 & : & 0 & 1 & : & 0 & : & 0 \\
\hline$H_{24} \mathcal{T}_{i}$ & $H_{24}$ & $\mathcal{T}_{j} H_{5}$ & $40, \overline{10}$ & 1 & $:$ & 1 & 1 & : & 1 & : & 0 \\
\hline$H_{24} \mathcal{T}_{i}$ & $H_{24}$ & $\mathcal{T}_{j} H_{5}$ & $40, \overline{40}$ & 1 & $:$ & $x$ & $: \quad-\frac{1}{2}$ & $:$ & $-2 x$ & : & 0 \\
\hline$H_{24} \mathcal{T}_{i}$ & $H_{24}$ & $\mathcal{T}_{j} H_{5}$ & $175, \overline{10}$ & 1 & $:$ & $\frac{64}{61}$ & 1 & $:$ & $\frac{64}{61}$ & : & $\frac{72}{61}$ \\
\hline$H_{24} \mathcal{T}_{i}$ & $H_{24}$ & $\mathcal{T}_{j} H_{5}$ & $175, \overline{40}$ & 1 & $:$ & 4 & $: \quad-\frac{1}{2}$ & : & -8 & : & 0 \\
\hline
\end{tabular}

Table 15. Continuation of table 14: $Y_{T T}$-like CG ratios for the effective dimension 6 operators $W \supset(A B)_{R_{1}} C(D E)_{R_{2}}$ corresponding to the the right diagram in figure 9. Note two combinations have a free parameter $x$. See main text for more details.

and

$$
C_{a}^{u, d, e}=\left(\begin{array}{ccc}
\frac{26}{5} & \frac{14}{5} & \frac{18}{5} \\
6 & 6 & 2 \\
4 & 4 & 0
\end{array}\right)
$$

where the first column stands for $u$, the second column for $d$ and the third for $e$. Additional colour triplet and weak doublet pairs, as those contained in $\mathbf{5}, \overline{\mathbf{5}}$ representations, contribute (per pair) at one-loop with

$$
b_{a}^{(5, T)}=\left(\begin{array}{c}
\frac{2}{5} \\
0 \\
1
\end{array}\right), \quad b_{a}^{(5, D)}=\left(\begin{array}{c}
\frac{3}{5} \\
1 \\
0
\end{array}\right)
$$


and at two-loop with

$$
B_{a b}^{(5, T)}=\left(\begin{array}{ccc}
\frac{8}{75} & 0 & \frac{32}{15} \\
0 & 0 & 0 \\
\frac{4}{15} & 0 & \frac{34}{3}
\end{array}\right), \quad B_{a b}^{(5, D)}=\left(\begin{array}{ccc}
\frac{9}{25} & \frac{9}{5} & 0 \\
\frac{3}{5} & 7 & 0 \\
0 & 0 & 0
\end{array}\right)
$$

$\mathrm{SU}(2)$ triplets, $\mathrm{SU}(3)$ octets and leptoquark superfields ${ }^{14}$ from an adjoint contribute (per chiral superfield) at one-loop with

$$
b_{a}^{(24, T)}=\left(\begin{array}{l}
0 \\
2 \\
0
\end{array}\right), \quad b_{a}^{(24, O)}=\left(\begin{array}{c}
0 \\
0 \\
3
\end{array}\right), \quad b_{a}^{(24, L)}=\left(\begin{array}{c}
\frac{5}{2} \\
\frac{3}{2} \\
1
\end{array}\right)
$$

and at two-loop with

$$
B_{a b}^{(24, T)}=\left(\begin{array}{ccc}
0 & 0 & 0 \\
0 & 24 & 0 \\
0 & 0 & 0
\end{array}\right), \quad B_{a b}^{(24, O)}=\left(\begin{array}{ccc}
0 & 0 & 0 \\
0 & 0 & 0 \\
0 & 0 & 54
\end{array}\right), \quad B_{a b}^{(24, L)}=\left(\begin{array}{ccc}
\frac{25}{6} & \frac{15}{2} & \frac{40}{3} \\
\frac{5}{2} & \frac{21}{2} & 8 \\
\frac{5}{3} & 3 & \frac{34}{3}
\end{array}\right) .
$$

Additional Yukawa couplings between SM fermion superfields and a pair of colour triplet/anti-triplet contribute with

$$
C_{a}^{q q, u e, q l, u d}=\left(\begin{array}{cccc}
\frac{6}{5} & \frac{28}{5} & \frac{14}{5} & \frac{24}{5} \\
6 & 0 & 6 & 0 \\
6 & 2 & 4 & 6
\end{array}\right)
$$

In our numerical analysis we have assumed that $Y_{q q}=Y_{u e}=Y_{u}$ and $Y_{q l}=Y_{u d}=Y_{d}$ (as motivated by minimal SU(5)). We have checked that this approximation changes our results only negligibly.

\section{Discussion of messenger fields}

We discuss now the messenger fields appearing in figure 6 of the model presented in section 4.1. There are three supergraphs generating the superpotential term $y_{12}$. The renormalizable superpotential corresponding to figure 6 is

$$
\begin{aligned}
W \supset & \gamma_{1} H_{5} \mathcal{T}_{1} Z_{10,4}+\gamma_{2} H_{5} \mathcal{T}_{2} Z_{10,3}+\gamma_{3} \mathcal{T}_{3} \theta_{1} \bar{Z}_{10,3}+\gamma_{\theta} \theta_{1}^{2} Z_{1} \\
& +\lambda_{1} \theta_{2} Z_{2} \bar{Z}_{1}+\lambda_{10} \theta_{2} Z_{10,3} \bar{Z}_{10,4} \\
& +\eta_{1} \mathcal{T}_{1} \bar{Z}_{10,3} \bar{Z}_{2}+\eta_{2} \mathcal{T}_{2} \bar{Z}_{10,4} \bar{Z}_{2}+\eta_{2}^{\prime} \mathcal{T}_{2} \bar{Z}_{10,3} \bar{Z}_{1} \\
& +M_{1} Z_{1} \bar{Z}_{1}+M_{2} Z_{2} \bar{Z}_{2}+M_{10,3} Z_{10,3} \bar{Z}_{10,3}+M_{10,4} Z_{10,4} \bar{Z}_{10,4}
\end{aligned}
$$

where we explicitly denote the coupling constants at the ends of a diagram with $\gamma_{x}$, coupling constants in the middle of the diagrams with $\lambda_{x}$ if they involve $\theta_{j}$ and $\eta_{i}$ if they involve

\footnotetext{
${ }^{14}$ Note that leptoquark superfields can only appear in Dirac pairs due to their charges.
} 
$\mathcal{T}_{i}$ and messenger masses with $M_{x}$. After $Z_{x}$ and $\bar{Z}_{x}$ are integrated out and the flavons $\theta_{i}$ obtain VEVs, the elements of the up-type Yukawa matrix $Y_{u}$ of eq. (4.2) are given by

$$
\begin{aligned}
y_{11} & =\gamma_{1} \lambda_{10} \eta_{1} \lambda_{1} \gamma_{\theta} \frac{\left\langle\theta_{1}\right\rangle^{2}\left\langle\theta_{2}\right\rangle^{2}}{M_{1} M_{2} M_{10,3} M_{10,4}}, \\
y_{12} & =\gamma_{2} \eta_{1} \lambda_{1} \gamma_{\theta} \frac{\left\langle\theta_{1}\right\rangle^{2}\left\langle\theta_{2}\right\rangle}{M_{1} M_{2} M_{10,3}}+\gamma_{1} \eta_{2} \lambda_{1} \gamma_{\theta} \frac{\left\langle\theta_{1}\right\rangle^{2}\left\langle\theta_{2}\right\rangle}{M_{1} M_{2} M_{10,4}}+\gamma_{1} \lambda_{10} \eta_{2}^{\prime} \gamma_{\theta} \frac{\left\langle\theta_{1}\right\rangle^{2}\left\langle\theta_{2}\right\rangle}{M_{1} M_{10,3} M_{10,4}} \\
y_{22} & =\gamma_{2} \eta_{2}^{\prime} \gamma_{\theta} \frac{\left\langle\theta_{1}\right\rangle^{2}}{M_{1} M_{10,3}}, \\
y_{13} & =\gamma_{1} \lambda_{10} \gamma_{3} \frac{\left\langle\theta_{1}\right\rangle\left\langle\theta_{2}\right\rangle}{M_{10,3} M_{10,4}}, \\
y_{23} & =\gamma_{2} \gamma_{3} \frac{\left\langle\theta_{1}\right\rangle}{M_{10,3}}
\end{aligned}
$$

and $y_{33}$ is a renormalizable Yukawa coupling coefficient.

Removing the messenger pair $Z_{2}, \bar{Z}_{2}$ from the spectrum eliminates two supergraphs and thus the first two terms contributing to $y_{12} \cdot{ }^{15}$ However, without $Z_{2} \bar{Z}_{2}$, evaluating $y_{12}$ yields

$$
\begin{aligned}
y_{12} & =\gamma_{1} \lambda_{10} \eta_{2}^{\prime} \gamma_{\theta} \frac{\left\langle\theta_{1}\right\rangle^{2}\left\langle\theta_{2}\right\rangle}{M_{1} M_{10,3} M_{10,4}}=y_{13} \frac{\left\langle\theta_{1}\right\rangle}{M_{1}} \frac{\eta_{2}^{\prime} \gamma_{\theta}}{\gamma_{3}} \\
& =y_{13} y_{22} \frac{M_{10,3}}{\left\langle\theta_{1}\right\rangle} \frac{1}{\gamma_{2} \gamma_{3}}=\frac{y_{13} y_{22}}{y_{23}} .
\end{aligned}
$$

This relation is not phenomenologically viable as it would imply $\theta_{C}=\theta_{13} / \theta_{23}$. To fit $Y_{u}$ to the observed data an additional degree of freedom is needed. In our model this is realised through $Z_{2} \bar{Z}_{2}$ enabling additional diagrams contributing to $y_{12}$.

\section{Simultaneous R-symmetry and GUT breaking}

It was shown in the literature that the MSSM with an additional R-symmetry cannot be obtained from the spontaneous breaking of a four-dimensional (SUSY) GUT [27]. On the other hand, in flavour models, R-symmetries are often used in superpotentials that generate the required VEVs for the family symmetry breaking Higgs fields (i.e. the flavons) as well as for spontaneous breaking of CP. In the following we present some simple examples that show that it is possible - in particular with discrete R-symmetries - to simultaneously break the GUT gauge group and the R-symmetry. Without an R-symmetry below the Planck scale, there is no conflict to the statement of [27]. We will also illustrate that GUT flavour models can rely on such a discrete R-symmetry for the flavon VEV alignment, such that our setup can be used to construct flavour models with non-Abelian family symmetries and spontaneous $\mathrm{CP}$ violation.

\footnotetext{
${ }^{15} \mathrm{~A}$ different pair of fields $Z_{2}^{\prime}, \bar{Z}_{2}^{\prime}$ would need to be introduced anyway (using different charge assignment than $Z_{2}, \bar{Z}_{2}$ ) in order to generate $y_{11}$. The charges can be assigned such that no extra contributions to $y_{12}$ appear.
} 
Simple example. Consider as example a discrete $\mathbb{Z}_{4}^{R}$ R-symmetry under which the superfield $S$ is charged and the superfield $H$ is uncharged (for the beginning we assume them to be $\mathrm{SU}(5)$ singlets). The fields are also charged under an additional conventional $\mathbb{Z}_{4}$ symmetry with charges 1 and 3 respectively. We consider only the required lowest order superpotential terms:

$$
W_{R}=\mu_{1} S H+\lambda_{1} S^{3} H^{3} / M_{\mathrm{Pl}}^{3}+\lambda_{2} S H^{5} / M_{\mathrm{Pl}}^{3}+\lambda_{3} S^{5} H / M_{\mathrm{Pl}}^{3} .
$$

The F-terms for this simple example lead to the conditions

$$
\begin{gathered}
\mu_{1} H+3 \lambda_{1} S^{2} H^{3} / M_{\mathrm{Pl}}^{3}+\lambda_{2} H^{5} / M_{\mathrm{Pl}}^{3}+5 \lambda_{3} S^{4} H / M_{\mathrm{Pl}}^{3}=0 \\
\mu_{1} S+3 \lambda_{1} S^{3} H^{2} / M_{\mathrm{Pl}}^{3}+5 \lambda_{2} S H^{4} / M_{\mathrm{Pl}}^{3}+\lambda_{3} S^{5} / M_{\mathrm{Pl}}^{3}=0 .
\end{gathered}
$$

These equations have several solutions but here we are only interested in the non-trivial solution $\langle S\rangle^{4}=\left(\lambda_{2} / \lambda_{3}\right)\langle H\rangle^{4}$ and $\langle H\rangle^{4}=-\mu_{1} M_{\mathrm{Pl}}^{3} /\left(3 \lambda_{1} \sqrt{\lambda_{2} / \lambda_{3}}+6 \lambda_{2}\right)$. If we assign $S$ and $H$ under $\mathrm{SU}(5)$ as adjoints (similarly to superpotential (c) where we had the two fields $H_{24}$ and $H_{24}^{\prime}$ ) $W_{R}$ is $\mathrm{SU}(5)$ invariant (by taking the appropriate contractions) and importantly, the R-symmetry and SU(5) are simultaneously broken by the non-trivial VEV configuration. We can achieve a phenomenological viable model by breaking a discrete Rsymmetry at the GUT scale.

In general, leaving additional symmetry (or symmetries) unspecified, $S$ may be a singlet of the GUT symmetry group and we denote the (polynomial) functions of the superfield $H$ that make the respective terms invariant as $A(H), C(H)$ (which include the associated couplings and $M_{\mathrm{Pl}}$ suppressions). We write

$$
W_{R}=S A(H)+S^{3} C(H),
$$

leading to the F-term equations

$$
\begin{aligned}
A(H)+3 C(H) S^{2} & =0, \\
\frac{\mathrm{d} A(H)}{\mathrm{d} H} S+\frac{\mathrm{d} C(H)}{\mathrm{d} H} S^{3} & =0 .
\end{aligned}
$$

The generalised solution breaking the R-symmetry and the GUT gauge group is then

$$
\begin{aligned}
\langle S\rangle^{2} & =-\left\langle\frac{A(H)}{3 C(H)}\right\rangle, \\
\left\langle\frac{\mathrm{d} A(H)}{\mathrm{d} H}\right\rangle & =\left\langle\frac{A(H)}{3 C(H)} \frac{\mathrm{d} C(H)}{\mathrm{d} H}\right\rangle .
\end{aligned}
$$

Note the second equation, $\frac{\mathrm{d} A}{A}=\frac{\mathrm{d} C}{3 C}$, constrains the allowed functions $A(H)$ and $C(H)$ which indirectly imposes conditions on the unspecified additional symmetries.

Generalisations. While the R-symmetry must be discrete for the crucial interplay between two terms, it needs not be a $\mathbb{Z}_{4}^{R}$ and generalising to other $\mathbb{Z}_{N}^{R}$ symmetries is straightforward

$$
W_{R}^{N}=S A(H)+S^{N-1} C(H)
$$


This can be further generalised to multiple fields. Consider, for instance, $S, R$ and $T$ charged under a $\mathbb{Z}_{4}^{R}$, some unspecified symmetries with fields $H, I, J$, and generalized functions $A_{S, R, T}(H, I, J)$ as well as $C(H, I, J)$, such that keeping only the necessary lowest order terms in $S, R, T$ we write

$$
W_{R}=S A_{S}(H, I, J)+R A_{R}(H, I, J)+T A_{T}(H, I, J)+S R T C(H, I, J) .
$$

After some manipulation we find again a non-trivial solution breaking $\mathbb{Z}_{4}^{R}$ and the GUT symmetry

$$
\frac{A_{S}}{\langle R T\rangle}=\frac{A_{R}}{\langle T S\rangle}=\frac{A_{T}}{\langle S R\rangle}=-C,
$$

provided the derivatives with respect to each of the GUT superfields $H, I, J$ fulfill

$$
\frac{\partial A_{S}}{A_{S}}+\frac{\partial A_{R}}{A_{R}}+\frac{\partial A_{T}}{A_{T}}+\frac{\partial C}{C}=0 .
$$

The simple examples above illustrate that a simultaneous breaking of R- and GUT symmetry is possible. VEV alignments required by flavour models can therefore still be obtained within GUTs by having additional superfields charged under the R-symmetry.

Flavour alignment. As a very simple example we discuss the "alignment" of a GUT singlet flavon $\phi$ charged under a non-Abelian family symmetry via a driving field $P$. As additional symmetries we impose $\mathbb{Z}_{4}^{R}$ and a conventional $\mathbb{Z}_{n}$. The allowed renormalizable superpotential is

$$
W=P\left(\frac{\phi^{n}}{\Lambda^{n-2}}+M^{2}\right)+\kappa P^{3},
$$

where $\Lambda$ is a generic messenger scale and $M$ a mass parameter. Minimising the F-term conditions we find two possible solutions:

$$
\begin{aligned}
& \text { solution A: }\langle P\rangle=0 \text { and }\langle\phi\rangle^{n}=-M^{2} \Lambda^{n-2}, \\
& \text { solution B: }\langle P\rangle^{2}=-\frac{M^{2}}{3 \kappa} \text { and }\langle\phi\rangle=0 .
\end{aligned}
$$

In a flavour model we want the flavon to get a non-vanishing VEV so that we would adopt solution A there. Furthermore solution A shows how the discrete vacuum alignment method [44] can be generalised to models with discrete R-symmetries. This method was invented in the context of spontaneous $\mathrm{CP}$ violation. If $\mathrm{CP}$ is promoted to be fundamental all the phases of the parameters in eq. (D.13) are fixed and hence the phase of the flavon VEV is fixed as well (up to a discrete choice). 
Open Access. This article is distributed under the terms of the Creative Commons Attribution License (CC-BY 4.0), which permits any use, distribution and reproduction in any medium, provided the original author(s) and source are credited.

\section{References}

[1] Z. Berezhiani and Z. Tavartkiladze, Anomalous U(1) symmetry and missing doublet SU(5) model, Phys. Lett. B 396 (1997) 150 [hep-ph/9611277] [INSPIRE].

[2] G. Altarelli, F. Feruglio and I. Masina, From minimal to realistic supersymmetric SU(5) grand unification, JHEP 11 (2000) 040 [hep-ph/0007254] [INSPIRE].

[3] H. Georgi and C. Jarlskog, A new lepton - quark mass relation in a unified theory, Phys. Lett. B 86 (1979) 297 [INSPIRE].

[4] H. Murayama, Y. Okada and T. Yanagida, The Georgi-Jarlskog mass relation in a supersymmetric grand unified model, Prog. Theor. Phys. 88 (1992) 791 [InSPIRE].

[5] D.-X. Zhang and J.-h. Zheng, A missing partner model with 24-plet breaking SU(5), JHEP 12 (2012) 087 [arXiv:1212.5852] [INSPIRE].

[6] H. Murayama and A. Pierce, Not even decoupling can save minimal supersymmetric SU(5), Phys. Rev. D 65 (2002) 055009 [hep-ph/0108104] [INSPIRE].

[7] A. Masiero, D.V. Nanopoulos, K. Tamvakis and T. Yanagida, Naturally massless Higgs doublets in supersymmetric SU(5), Phys. Lett. B 115 (1982) 380 [InSPIRE].

[8] B. Grinstein, A supersymmetric $\mathrm{SU}(5)$ gauge theory with no gauge hierarchy problem, Nucl. Phys. B 206 (1982) 387 [InSPIRE].

[9] J. Hisano, T. Moroi, K. Tobe and T. Yanagida, Suppression of proton decay in the missing partner model for supersymmetric SU(5) GUT, Phys. Lett. B 342 (1995) 138 [hep-ph/9406417] [INSPIRE].

[10] Particle Data Group collaboration, J. Beringer et al., Review of particle physics, Phys. Rev. D 86 (2012) 010001 [InSPIRE].

[11] S. Antusch and M. Spinrath, New GUT predictions for quark and lepton mass ratios confronted with phenomenology, Phys. Rev. D 79 (2009) 095004 [arXiv:0902.4644] [INSPIRE].

[12] S. Antusch and V. Maurer, Large neutrino mixing angle $\theta_{13}^{\mathrm{MNS}}$ and quark-lepton mass ratios in unified flavour models, Phys. Rev. D 84 (2011) 117301 [arXiv:1107.3728] [INSPIRE].

[13] D. Marzocca, S.T. Petcov, A. Romanino and M. Spinrath, Sizeable $\theta_{13}$ from the charged lepton sector in $\mathrm{SU}(5)$, (tri-)bimaximal neutrino mixing and Dirac CP-violation, JHEP 11 (2011) 009 [arXiv:1108.0614] [INSPIRE].

[14] S. Antusch, C. Gross, V. Maurer and C. Sluka, $\theta_{13}^{\text {PMNS }}=\theta_{C} / \sqrt{2}$ from GUTs, Nucl. Phys. B 866 (2013) 255 [arXiv:1205.1051] [INSPIRE].

[15] A. Meroni, S.T. Petcov and M. Spinrath, A SUSY SU(5) $\times T^{\prime}$ unified model of flavour with large $\theta_{13}$, Phys. Rev. D 86 (2012) 113003 [arXiv:1205.5241] [INSPIRE].

[16] S. Antusch, C. Gross, V. Maurer and C. Sluka, A flavour GUT model with $\theta_{13}^{\text {PMNS }} \simeq \theta_{C} / \sqrt{2}$, Nucl. Phys. B 877 (2013) 772 [arXiv:1305.6612] [INSPIRE]. 
[17] S. Antusch, C. Gross, V. Maurer and C. Sluka, Inverse neutrino mass hierarchy in a flavour GUT model, Nucl. Phys. B 879 (2014) 19 [arXiv:1306.3984] [InSPIRE].

[18] T. Goto and T. Nihei, Effect of RRRR dimension five operator on the proton decay in the minimal SU(5) SUGRA GUT model, Phys. Rev. D 59 (1999) 115009 [hep-ph/9808255] [INSPIRE].

[19] P. Nath and P. Fileviez Perez, Proton stability in grand unified theories, in strings and in branes, Phys. Rept. 441 (2007) 191 [hep-ph/0601023] [INSPIRE].

[20] I. de Medeiros Varzielas and L. Merlo, Ultraviolet completion of flavour models, JHEP 02 (2011) 062 [arXiv: 1011.6662] [INSPIRE].

[21] I. de Medeiros Varzielas and D. Pidt, UV completions of flavour models and large $\theta_{13}$, JHEP 03 (2013) 065 [arXiv:1211.5370] [INSPIRE].

[22] S. Antusch, S.F. King and M. Spinrath, GUT predictions for quark-lepton Yukawa coupling ratios with messenger masses from non-singlets, Phys. Rev. D 89 (2014) 055027 [arXiv: 1311.0877] [INSPIRE].

[23] S. Dimopoulos and H. Georgi, Softly broken supersymmetry and SU(5), Nucl. Phys. B 193 (1981) 150 [inSPIRE].

[24] B. Bajc, P. Fileviez Perez and G. Senjanović, Minimal supersymmetric SU(5) theory and proton decay: where do we stand?, hep-ph/0210374 [INSPIRE].

[25] S. Antusch and V. Maurer, Running quark and lepton parameters at various scales, JHEP 11 (2013) 115 [arXiv:1306.6879] [INSPIRE].

[26] L.J. Hall, Grand unification of effective gauge theories, Nucl. Phys. B 178 (1981) 75 [INSPIRE].

[27] M. Fallbacher, M. Ratz and P.K.S. Vaudrevange, No-go theorems for $R$ symmetries in four-dimensional GUTs, Phys. Lett. B 705 (2011) 503 [arXiv:1109.4797] [INSPIRE].

[28] L.J. Hall, R. Rattazzi and U. Sarid, The top quark mass in supersymmetric $\mathrm{SO}(10)$ unification, Phys. Rev. D 50 (1994) 7048 [hep-ph/9306309] [INSPIRE].

[29] M.S. Carena, M. Olechowski, S. Pokorski and C.E.M. Wagner, Electroweak symmetry breaking and bottom-top Yukawa unification, Nucl. Phys. B 426 (1994) 269 [hep-ph/9402253] [INSPIRE].

[30] R. Hempfling, Yukawa coupling unification with supersymmetric threshold corrections, Phys. Rev. D 49 (1994) 6168 [inSPIRE].

[31] T. Blazek, S. Raby and S. Pokorski, Finite supersymmetric threshold corrections to CKM matrix elements in the large $\tan \beta$ regime, Phys. Rev. D 52 (1995) 4151 [hep-ph/9504364] [INSPIRE].

[32] S. Antusch, L. Calibbi, V. Maurer and M. Spinrath, From flavour to SUSY flavour models, Nucl. Phys. B 852 (2011) 108 [arXiv:1104.3040] [InSPIRE].

[33] S.F. King, Minimal predictive see-saw model with normal neutrino mass hierarchy, JHEP 07 (2013) 137 [arXiv: 1304.6264] [INSPIRE].

[34] S.F. King, A model of quark and lepton mixing, JHEP 01 (2014) 119 [arXiv:1311.3295] [INSPIRE].

[35] S. Antusch, S.F. King, M. Malinsky and M. Spinrath, Quark mixing sum rules and the right unitarity triangle, Phys. Rev. D 81 (2010) 033008 [arXiv:0910.5127] [INSPIRE]. 
[36] P.Z. Skands et al., SUSY Les Houches accord: interfacing SUSY spectrum calculators, decay packages and event generators, JHEP 07 (2004) 036 [hep-ph/0311123] [INSPIRE].

[37] D. Emmanuel-Costa and S. Wiesenfeldt, Proton decay in a consistent supersymmetric SU(5) GUT model, Nucl. Phys. B 661 (2003) 62 [hep-ph/0302272] [InSPIRE].

[38] S. Wiesenfeldt, Proton decay in supersymmetric GUT models, Mod. Phys. Lett. A 19 (2004) 2155 [hep-ph/0407173] [INSPIRE].

[39] P. Nath, Hierarchies and textures in supergravity unification, Phys. Rev. Lett. 76 (1996) 2218 [hep-ph/9512415] [INSPIRE].

[40] V. Lucas and S. Raby, Nucleon decay in a realistic $\mathrm{SO}(10)$ SUSY GUT, Phys. Rev. D 55 (1997) 6986 [hep-ph/9610293] [INSPIRE].

[41] M. Spinrath, New aspects of flavour model building in supersymmetric grand unification, arXiv: 1009.2511 [INSPIRE].

[42] D.R.T. Jones, Asymptotic behavior of supersymmetric Yang-Mills theories in the two loop approximation, Nucl. Phys. B 87 (1975) 127 [INSPIRE].

[43] D.R.T. Jones and L. Mezincescu, The $\beta$-function in supersymmetric Yang-Mills theory, Phys. Lett. B 136 (1984) 242 [INSPIRE].

[44] S. Antusch, S.F. King, C. Luhn and M. Spinrath, Right unitarity triangles and tri-bimaximal mixing from discrete symmetries and unification, Nucl. Phys. B 850 (2011) 477 [arXiv:1103.5930] [INSPIRE]. 